\title{
REPRESENTATION OF FINITE GROUPS
}

\author{
MANSI BAJAJ
}

under the supervision of

Dr. Venku Naidu Dogga

A thesis submitted to

Indian Institute of Technology, Hyderabad

In Partial Fulfillment of the Requirement for

The Degree of Master of Science in Mathematics

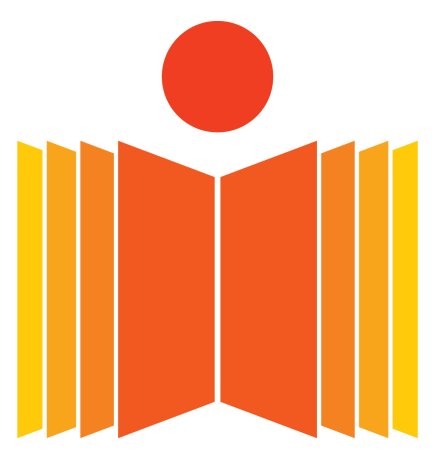

भारतीय प्रौद्योगिकी संस्थान हैदराबाद Indian Institute of Technology Hyderabad

\section{Department of Mathematics Indian Institute of Technology, Hyderabad Telangana-502285 \\ May 2019}




\section{DECLARATION}

This thesis entitled REPRESENTATION OF FINITE GROUPS submitted by me to the Indian Institute of Technology, Hyderabad for the award of the degree of Master of Science in Mathematics contains a literature survey of the work done by some authors in this area. The work presented in this thesis has been carried out under the supervision of Dr. Venku Naidu Dogga, Department of Mathematics, Indian Institute of Technology, Hyderabad, Telangana.

I hereby declare that, to the best of my knowledge, the work included in this thesis has been taken from the books, "Representation of finite and compact groups" by Barry Simon, "Representation of finite groups" by Benjamin Steinberg, and "Linear Representations of finite groups" by Jean Pierre Serre. No new results have been created in this thesis. The definitions, notations and results in representation theory are learnt from the above mentioned sources and are presented here. I have adequately cited and referenced the original sources. I also declare that I have adhered to all principles of academic honesty and integrity and have not misrepresented or fabricated or falsified any idea/data/fact/source in my submission. I understand that any violation of the above will be a cause for disciplinary action by the Institute and can also evoke penal action from the sources that have thus not been properly cited, or from whom proper permission has not been taken when needed.

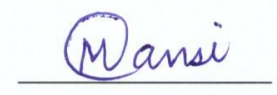

(Signature)

MANSI BAJAJ

(Mansi Bajaj)

MA17MSCST1 1008

(Roll No.) 


\section{Approval Sheet}

This Thesis entitled Representation of finite groups by Mansi Bajaj is approved for the degree of Master of Science from IIT Hyderabad.

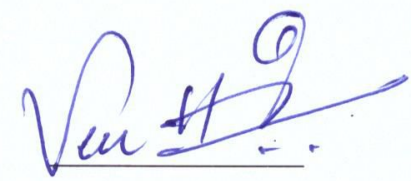

(Dr. Venku Naidu Dogga) Adviser Dept. of Mathematics IITH 


\section{ACKNOWLEDGEMENTS}

I would like to express my deep sense of gratitude to my supervisor, $D r$. Venku Naidu Dogga, for his constant encouragement, co-operation and invaluable guidance throughout this project. Due to his motivation and expert guidance, I was able to understand the concepts in a nice manner. I am thankful to Dr. Balasubramaniam Jayaram, Head of the Department during my M.Sc. Programme, for being understanding and supporting.

I thank the teachers of the department for imparting in me the knowledge and understanding of mathematics. Without their kind efforts I would not have reached this stage.

I would also like to extend my gratitude to my family and friends for helping me in every possible way and encouraging me during this Programme. Above all, I thank, The Almighty, for all his blessings.

\section{Mansi Bajaj}





\section{ABSTRACT}

The main aim of this project is to learn a branch of Mathematics that studies abstract algebraic structures by representating their elements as linear transformations of vector spaces. This branch is known as Representation Theory. A representation makes an abstract algebraic object more concrete by describing its elements by matrices and the algebraic operations in terms of matrix addition and matix multiplication. The most prominent is the representation theory of groups which is discussed in this thesis.

In representation of groups, elements of group are represented by invertible elements in such a way that the group operation is matrix multiplication. If say, $\mathrm{R}$ is a representation of a group $\mathrm{G}$ on vector space $\mathrm{V}$, then $\mathrm{V}$ is called as the representation space for G. There are two ways to describe a representation. First idea is to use action. That is, representation of a group $\mathrm{G}$ on a vector space $\mathrm{V}$ is a map $\phi: G \times V \rightarrow V$ with property that $\phi_{g}: V \rightarrow V$ defined as $v \longmapsto \phi(g, v)$ is linear over the field $\mathbb{F}$. Further, if $\phi(g, v)=g \cdot v$ then the properties of a group action hold for any elements $g_{1}, g_{2} \in G$ and $v \in V$. The second way to define a representation focuses on map $\phi$ defined as $\phi(g): V \rightarrow V$ to be a homomorphism. This approach is more concise and hence is being used in this project.

Moreover, the irreducible representations play an important role in Representation theory since every finite dimensional representation is a direct sum of irreducible representations which is an important result known as Maschke's theorem in this theory. This theory has some extremely useful fundamental results. One of them is Schur's lemma which says that if we have two irreducible representations $\phi$ and $\psi$ of a finite group G on vector spaces $\mathrm{V}$ and $\mathrm{W}$ respectively and $\mathrm{T}$, a linear transformation from $\mathrm{V}$ to $\mathrm{W}$ that commutes with action of group, then $\mathrm{T}$ is either invertible or is zero. This lemma is named after Issai Schur who used it to prove Schur's Orthogonality Relations and develop the basics of Representation theory of finite groups. Schur's lemma admits generalizations to Lie groups and Lie Algebras but this part is not mentioned in this thesis. 
Further, the Dimension theorem is proved here which states that degree of any irreducible representation of a group divides the order of that group. Then, using this theorem, again the most fundamental result of group theory known as the Burnside's lemma is proved using Representation theory. It states that no finite abelian group of order $p^{a} q^{b}$ where $\mathrm{p}, \mathrm{q}$ are primes and $\mathrm{a}, \mathrm{b}$ are non negative, is simple. Apart from these results, the permutation representations, regular representations and fourier analysis of finite groups have also been discussed here in this project. 


\section{Preface}

\section{Motivation}

In the mathematical field of representation theory, group representations describe abstract groups in terms of linear transformations of vector spaces. In particular, they can be used to represent group elements as matrices so that the group operation can be represented by matrix multiplication.

Representations of groups are important because they allow many theoretical problems to be reduced to problems in linear algebra which is well understood. They are also important in physics because, for example, they describe how the symmetry group of physical system affects the solutions of equations describing that system. The term 'representation of a group' is also used in a more general sense to mean any description of a group as a group of transfomations of some mathematical object. Or we can say that, a representation means a homomorphism from the group to automorphism group of an object.If this object is a vector space, we have a linear representation.

This thesis is divided into five chapters. Chapter one consists of the definitions , notations, some fundamental results of Representation theory, the irreducible representations, Schur's lemma and ends up with a short introduction to Tensor products. Chapter two consists of the Orthogonality Relations, Characters, class functions, the Regular representation and Representation of finite abelian groups. Chapter three contains some results of fourier analysis on finite groups. Chapter four describes the most fundamental result in Representation Theory, The Burnside's theorem. It uses some results from field theory to prove this theorem. Chapter five talks about the group actions and the permutation representations ending up with the introduction and some representation related results of Centralizer Algebra and the Gelfand Pair. 


\section{Contents}

$\begin{array}{ll}\text { Preface } & \text { ii }\end{array}$

1 Introduction to Representation 1

1.1 Definition and Unitarity . . . . . . . . . . . . . . . 1

1.2 Irreducibility and Complete Reduction . . . . . . . . . 8

1.3 The Group Algebra and the Regular Representations . . . . . 13

1.4 Schur's Lemma . . . . . . . . . . . . . . . . . . . . . 24

1.5 Tensor Products . . . . . . . . . . . . . . . . . . . 25

2 Orthogonality Relations 29

2.1 Morphisms . . . . . . . . . . . . . . . . . . . . 29

2.2 The Orthogonality Relations . . . . . . . . . . . . . . . . . . 29

2.3 Schur Orthogonality Relations . . . . . . . . . . . . . . . . 33

2.4 Characters and Class functions . . . . . . . . . . . 34

2.5 The Regular Representation . . . . . . . . . . . . . . . . . . 39

2.6 Representation of Finite Abelian Groups . . . . . . . . . . 45

3 Fourier Analysis on Groups $\quad 47$

3.1 Fourier Analysis on Finite Groups _ . . . . . . . . . . . 47

3.2 Fourier Analysis o finite abelian groups . . . . . . . . . . . 48

4 Burnside's theorem $\quad 55$

4.1 Number Theory . . . . . . . . . . . . . . . . . . . . 55

4.2 The Dimension Theorem . . . . . . . . . . . . . . . 57

4.3 The Burnside's Theorem . . . . . . . . . . . . . . . . 61

5 Group Actions and the Permutation Representations $\quad \mathbf{6 7}$ 5.1 Group Actions . . . . . . . . . . . . . . 67 
5.2 The Centralizer Algebra . . . . . . . . . . . . . . . . 74

5.3 Gelfand Pair . . . . . . . . . . . . . . . . . . 77 


\section{Chapter 1}

\section{Introduction to Representation}

\subsection{Definition and Unitarity}

Let $\mathrm{V}$ be a finite dimensional vector space. Let $\operatorname{Hom}(\mathrm{V})$ be the set of linear maps 'A' from 'V' to 'V'. For a pair of vectors, $V$ and $W$, let $\operatorname{Hom}(\mathrm{V}, \mathrm{W})$ be the space of linear maps from $\mathrm{V}$ to $\mathrm{W} . \mathrm{GL}(\mathrm{V}) \subset \mathrm{Hom}(\mathrm{V})$ are those maps A which are invertible. GL is known as the general group under composition with group identity $\mathrm{I}$, the identity matrix. If $\mathrm{V}$ is a real or complex vector space, GL(V) is precisely the set of continuous automorphisms(since any bounded linear operator is continuous) of $\mathrm{V}$ as a group under addition.

Definition 1.1.1. A group representation is a homomorphism of $G$ to $G L(V)$ for some ' $V$ '. The dimension of ' $V$ ' is called the degree of representation.

Example 1.1.2. Following are some well known group representations.

1. Trivial Representation: $\phi: G \rightarrow \mathbb{C}^{*}$ given by $\phi(g)=1 \forall g \in G$.

2. $\phi: \mathbb{Z} / n \mathbb{Z} \rightarrow \mathbb{C}^{*}$ given by

$$
\phi([m])=\exp (2 \pi i m / n)
$$

is also a representation.

Definition 1.1.3. Let $\phi: G \rightarrow G L(V)$ and $\psi: G \rightarrow G L(V)$ be two representations of a finite group $G$ on vector spaces $V$ and $W$ respectively. Then they are said to be equivalent if there exists an isomorphism $T: V \rightarrow W$ such that $\psi_{g}=T \phi_{g} T^{-} 1 \forall g \in G$, that is, $\psi_{g} T=T \phi_{g}, \forall g \in G$. 
Remark 1.1.4. The map $T$ is called the intertwining map for $\phi_{g}$ and $\psi_{g}$.

Example 1.1.5. Define $\phi: \mathbb{Z} / n \mathbb{Z} \rightarrow G L_{2}(\mathbb{C})$ by,

$$
\phi([m])=\left(\begin{array}{cc}
\cos \left(\frac{2 \pi i m}{n}\right) & -\sin \left(\frac{2 \pi i m}{n}\right) \\
\sin \left(\frac{2 \pi i m}{n}\right) & \cos \left(\frac{2 \pi i m}{n}\right)
\end{array}\right) .
$$

This matrix is known as the matrix of rotation by angle $\frac{2 \pi i m}{n}$ and define $\psi: \mathbb{Z} / n \mathbb{Z} \rightarrow G L_{2}(\mathbb{C})$ by

$$
\psi([m])=\left(\begin{array}{cc}
\exp \left(\frac{2 \pi i m}{n}\right) & 0 \\
0 & \exp \left(\frac{-2 \pi i m}{n}\right)
\end{array}\right) .
$$

Now, let

$$
A=\left(\begin{array}{cc}
i & -i \\
1 & 1
\end{array}\right)
$$

then,

$$
A^{-1}=\frac{1}{2 i}\left(\begin{array}{cc}
1 & i \\
-1 & i
\end{array}\right)
$$

so, $A^{-1} \phi([m]) A=$

$$
\begin{gathered}
\frac{1}{2 i}\left(\begin{array}{cc}
1 & i \\
-1 & i
\end{array}\right)\left(\begin{array}{cc}
\cos \left(\frac{2 \pi i m}{n}\right) & -\sin \left(\frac{2 \pi i m}{n}\right) \\
\sin \left(\frac{2 \pi i m}{n}\right) & \cos \left(\frac{2 \pi i m}{n}\right)
\end{array}\right)\left(\begin{array}{cc}
i & -i \\
1 & 1
\end{array}\right) \\
\left.=\frac{1}{2 i}\left(\begin{array}{c}
\exp \left(\frac{2 \pi i m}{n}\right) \\
-\exp \left(\frac{-2 \pi i m}{n}\right)
\end{array}\right) \exp \left(\frac{2 \pi i m}{n}\right)\right)\left(\begin{array}{cc}
i & -i \\
1 & 1
\end{array}\right) \\
=\frac{1}{2 i}\left(\begin{array}{c}
2 i \exp \left(\frac{2 \pi i m}{n}\right) \\
0
\end{array}\right) \\
2 i \exp \left(\frac{-2 \pi i m}{n}\right)
\end{gathered}
$$


Note 1.1.6. We consider complex vector spaces $V \cong \mathbb{C}^{n}$. An inner product on $V$ is a map $\langle.,\rangle:. V \times V \rightarrow \mathbb{C}$ such that,

1. $\langle v,$.$\rangle is linear, \forall$ fixed $v$.

2. $\langle w, v\rangle=\overline{\langle v, w\rangle}$.

3. $\langle v, v\rangle \geq 0$, with equality if and only if $v=0$.

Definition 1.1.7. A unitary operator is a surjective bounded operator on a Hilbert space preserving inner product. Or we can say that, A Unitary Operator is a bounded linear operator $\mathbb{U}: \mathbb{H} \rightarrow \mathbb{H}$ on a Hilbert space $\mathbb{H}$ that satisfies $\mathbb{U}^{*}=\mathbb{U}^{*} \mathbb{U}=\mathbb{I}$ where $\mathbb{I}: \mathbb{H} \rightarrow \mathbb{H}$ is the identity operator.

Theorem 1.1.8. Let $U: G \rightarrow G L(V)$ be a representation of a finite group $G$. Then, $V$ has an inner product $\langle.,$.$\rangle in which each U(g)$ is unitary i.e., $\langle U(g) v, U(g) w\rangle=\langle v, w\rangle \forall v, w \in V, g \in G$.

Proof. Let $\langle,\rangle_{0}$ be an inner product on $\mathrm{V}$.

Define $\langle v, w\rangle=\frac{1}{O(G)} \sum_{g \in G}\langle U(g) v, U(g) w\rangle_{0}$.

We need to show that $\langle v, w\rangle$ defined above is an inner product.

1. $\langle v, v\rangle \geq 0 \forall v \in V$.

2. By definition $\langle v, v\rangle=0$ if and only if we have the following.

$$
\begin{aligned}
\langle v, v\rangle=0 & \text { if and only if } \frac{1}{O(G)} \sum_{g \in G}\langle U(g) v, U(g) w\rangle_{0}=0 \\
& \text { if and only if }\langle U(g) v, U(g) w\rangle_{0}=0 \\
& \text { if and only if } U(g) v=0 \text { if and only if } \mathrm{v}=0 .
\end{aligned}
$$

3. We have,

$$
\begin{aligned}
\langle w, v\rangle & =\frac{1}{O(G)} \sum_{g \in G}\langle U(g) v, U(g) w\rangle_{0} \\
& =\frac{1}{O(G)} \sum_{g \in G} \overline{\langle U(g) v, U(g) w\rangle_{0}} \\
& =\overline{\langle v, w\rangle} .
\end{aligned}
$$


4. We have,

$$
\begin{aligned}
\langle u+v, w\rangle= & \frac{1}{O(G)} \sum_{g \in G}\langle U(g) u+U(g) v, U(g) w\rangle_{0} \\
& =\frac{1}{O(G)} \sum_{g \in G}\left[\langle U(g) u, U(g) w\rangle_{0}+\langle U(g) v, U(g) w\rangle_{0}\right] \\
& =\frac{1}{O(G)} \sum_{g \in G}\langle U(g) u, U(g) w\rangle_{0}+\frac{1}{O(G)} \sum_{g \in G}\langle U(g) v, U(g) w\rangle_{0} \\
& =\langle u, w\rangle+\langle v, w\rangle .
\end{aligned}
$$

Hence, $\langle.,$.$\rangle is an inner product on \mathrm{V}$.

Moreover,

$$
\begin{aligned}
\langle U(h) v, U(h) w\rangle & =\frac{1}{O(G)} \sum_{g \in G}\langle U(g) U(h) v, U(g) U(h) w\rangle_{0} \\
& =\frac{1}{O(G)} \sum_{g \in G}\langle U(g h) v, U(g h) w\rangle_{0} \quad \text { (since, U is homomorphism) } \\
& =\frac{1}{O(G)} \sum_{g \in G}\langle U(g) v, U(g) w\rangle_{0} \\
& =\langle v, w\rangle .
\end{aligned}
$$

since, for each fixed h, $g \longmapsto g h$ is a bijection, so as g runs through G, so does gh.

Example 1.1.9. Let $G=\mathbb{Z}$. Let $a \in \mathbb{C}^{*}$ be fixed and $U: G \rightarrow G L(\mathbb{C})$ be defined by

$$
U(n)=a^{n}
$$

If $|a| \neq 1, U$ is not unitary in the only inner product that $\mathbb{C}$ supports as, $\langle U(n) x, U(n) y\rangle=\left\langle a^{n} x, a^{n} y\right\rangle=\left|a^{n}\right|^{2}\langle x, y\rangle \neq\langle x, y\rangle$ if $|a| \neq 1$.

Therefore, it implies that each $U(g)$ has eigenvalues $\omega$ with

$$
|\omega|=1 \text {. }
$$

(since, $g^{n}$ for some $n \Rightarrow U(g)^{n}=\mathbb{I} \Rightarrow \omega^{n}=1$ that is, eigen values are roots of unity). 
Note 1.1.10. 1. Hilbert Space is a real or complex inner product space that is also a complete metric space with respect to the distance function induced by the inner product.

2. Unitary Representation of a group $G$ is a linear representation $\pi$ of $G$ on a complex Hilbert space $V$ such that $\pi(g)$ is a unitary operator $\forall g$ $\in G$.

Definition 1.1.11. Let $V$ be a finite dimensional complex space with inner product (hilbert space). $\mathbb{U}(V)$, the unitary operators on $V$ is a group( under composition).

$A$ unitary representation of a finite group $G$ is a homomorphism of $G$ to $\mathbb{U}(V)$ (when we say representation, we mean unitary representation).

Note 1.1.12. One way of rephrasing Thm 2.2 is:

If $U: G \rightarrow G L(V) ; V$ is a hilbert space and $G$ is finite, then there is a $T \in G L(V)$ such that $T U(g) T^{-1}$ is unitary.

Definition 1.1.13. Let $U: G \rightarrow \mathbb{U}(X), V: G \rightarrow \mathbb{U}(Y)$ be two representations of same group $G$, where, $\mathbb{U}(X)$ and $\mathbb{U}(Y)$ are unitary operators on $X$ and $Y$ respectively.

We say that $U$ and $V$ are unitarily equivalent $\Leftrightarrow$ there exist a unitary $W$ : $X \rightarrow Y$ such that $V(g)=W U(g) W^{-1} \forall g \in G$.

Note 1.1.14. We classify the representations upto equivalence.

Definition 1.1.15. A symmetric $n \times n$ real matrix $M$ is said to be positive definite if $z^{T} M z$ is strictly positive $\forall$ non zero column vector $z$ of $n$ real numbers.

Or

an $n \times n$ hermitian matrix $M$ is said to be positive definite if the scalar $z^{*} M z$ is strictly positive $\forall$ non zero column vector $z$ of $n$ complex numbers.

Note 1.1.16. 1) $z^{*} M z$ is automatically real since, $M$ is hermitian.

2) Entries on main diagonal of any hermitian matrix are real.

Definition 1.1.17. A matrix $M$ is called positive semi definite if $z^{T} M z$ or $z^{*} M z$ are positive or zero. 
Definition 1.1.18 (Polar Decomposition of a matrix:). Right polar decomposition of a matrix $A$ is, $A=U P$, where, $A \in C^{m \times n} ; m>=n$, $U \in C^{m \times n}$ with orthonormal columns and $P \in C^{n \times n}$ is positive definite.

Left polar decomposition of a matrix $A$ is, $A=P U$, where, $A \in C^{n \times m} ; m>=$ $n, U \in C^{n \times m}$ with orthonormal columns and $P \in C^{n \times n}$ is positive definite.

Remark 1.1.19. If $T=W|T|$ is a polar decomposition of $T$ then, $W$ is the partial isometry and $|T|$ is positive semi definite.

A partial isometry is a linear map between Hilbert spaces such that it is an isometry on the orthogonal complement of kernel.

If the kernel is $\{0\}$, then, in that case, partial isometry is unitary.

Theorem 1.1.20. Let $U, V$ be unitary representations of $G$ on $X$ and $Y$ respectively. Suppose, there exists an invertible map $T: X \rightarrow Y$ such that $U(g)=T^{-1} V(g) T, \forall g \in G$. Then, $\exists$ a unitary map $W: X \rightarrow Y$ such that $U(g)=W V(g) W^{-1}, \forall g \in G$.

Proof. Since U(g) is a unitary operator,

$$
U(g) U(g)^{*}=U(g)^{*} U(g)=\mathbb{I} .
$$

This implies,

$$
U\left(g^{*}\right)=U(g)^{-1}=U\left(g^{-1}\right)
$$

(since, $\mathrm{U}$ is a homomorphism).

Now, given that, $\exists$ an invertible map $T: X \rightarrow Y$ such that

$$
U(g)=T^{-1} V(g) T
$$

Taking adjoint and using $U(g)^{*}=U\left(g^{-1}\right)$, and replacing $\mathrm{g}$ by $g^{-1}$, we get,

$$
\begin{gathered}
U(g)^{*}=T^{*} V(g)^{*}\left(T^{-1}\right)^{*} \\
\text { i.e., } U(g)^{-1}=T^{*} V(g)^{-1}\left(T^{*}\right)^{-1} \\
\text { i.e., } U\left(g^{-1}\right)=T^{*} V(g)^{-1}\left(T^{*}\right)^{-1} \\
\text { i.e., } U(g)=T^{*} V(g)\left(T^{*}\right)^{-1}
\end{gathered}
$$


(since, true for all $\mathrm{g}$, in general)

$$
\begin{gathered}
\text { i.e., } V(g)=\left(T^{*}\right)^{-1} U(g) T^{*} \\
\text { i.e., } V(g)=T U(g) T^{-1} \text {. }
\end{gathered}
$$

Now,

$$
\begin{aligned}
T^{*} T U(g)\left(T^{*} T\right)^{-1} & =T^{*} T U(g)\left(T^{-1}\left(T^{*}\right)^{-1}\right) \\
& =T^{*}\left(T U(g) T^{-1}\right)\left(T^{*}\right)^{-1} \\
& =T^{*} U(g)\left(T^{*}\right)^{-1} \\
& =U(g) .
\end{aligned}
$$

This implies, $U(g)^{-1}\left(T^{*} T\right) U(g)=T^{*} T$. Now, by uniqueness of square root,

$$
U(g)^{-1}|T| U(g)=|T|
$$

Let $T=W|T|$ be the polar decomposition of $\mathrm{T}$, where $\mathrm{W}$ is unitary from $\mathrm{X}$ to $\mathrm{Y}$. Since, $|T|$ is invertible,

$$
\begin{aligned}
W U(g) W^{-1} & =T|T|^{-1} U(g)|T| T^{-1} \\
& =T U(g) T^{-1} \\
& =V(g) .
\end{aligned}
$$

Hence proved.

Note 1.1.21. In above theorem, $T$ is an invertible linear map (automorphism) $\Rightarrow \operatorname{Ker} T=\{0\}$, hence, $W$ is unitary.

Example 1.1.22. Standard Representation of $S_{n}$ Define $\phi: S_{n} \rightarrow$ $G L_{n}(\mathbb{C})$ on standard basis by

$$
\phi_{\sigma\left(e_{i}\right)}=e_{\sigma(i)}
$$

For instance, 


$$
\begin{aligned}
& \text { when } n=3, \phi(12)=\left(\begin{array}{lll}
0 & 1 & 0 \\
1 & 0 & 0 \\
0 & 0 & 1
\end{array}\right) \text { and } \phi(123)=\left(\begin{array}{lll}
0 & 0 & 1 \\
1 & 0 & 0 \\
0 & 1 & 0
\end{array}\right) \\
& \phi_{\sigma}\left(e_{1}+e_{2}+\ldots .+e_{n}\right)=e_{\sigma(1)}+e_{\sigma(2)}+\ldots .+e_{\sigma(n)}=e_{1}+e_{2}+\ldots+e_{n} \text {. }
\end{aligned}
$$

Thus, $\mathbb{C}\left(e_{1}+e_{2}+\ldots+e_{n}\right)$ is invariant under $\phi_{\sigma}$ with $\sigma \in S_{n}$.

\subsection{Irreducibility and Complete Reduction}

Definition 1.2.1. Let $X$ and $Y$ be vector spaces. Their Direct sum $X \oplus Y$ is defined as the cartesian product $X \times Y$ with coordinatewise operation.

For example, $\left(x_{1}, y_{1}\right)+\left(x_{2}, y_{2}\right)=\left(x_{1}+x_{2}, y_{1}+y_{2}\right)$.

Note: If $\mathrm{X}$ and $\mathrm{Y}$ are Hilbert spaces, so is $X \oplus Y$, with inner poduct as follows:

$$
\left\langle\left(x_{1}, y_{1}\right),\left(x_{2}, y_{2}\right)\right\rangle=\left\langle x_{1}, x_{2}\right\rangle_{X}+\left\langle y_{1}, y_{2}\right\rangle_{Y}
$$

Definition 1.2.2. If $A \in \operatorname{Hom}(X), B \in \operatorname{Hom}(Y)$ then, $A \oplus B \in \operatorname{Hom}(X \oplus$ Y) by:

$$
(A \oplus B)(x, y)=(A x, B y) .
$$

Also, $(A \oplus B)(C \oplus D)=(A C \oplus B D)$. And if, $A \in \mathbb{U}(X), B \in \mathbb{U}(Y)$, then,

$$
A \oplus B \in \mathbb{U}(X \oplus Y) .
$$

\section{Verification I:}

We need to show that $A \oplus B \in \operatorname{Hom}(X \oplus Y)$ if $A \in \operatorname{Hom}(X)$ and $B \in$ $\operatorname{Hom}(Y)$. That means to show $A \oplus B: X \oplus Y \rightarrow X \oplus Y$ is linear. That is, we show, $A \oplus B(\alpha x+y)=\alpha(A \oplus B)(x)+(A \oplus B)(y)$.

Consider, $(A \oplus B)(\alpha x+y) ; x, y \in X \oplus Y$.

Let $x=\left(x_{1}, x_{2}\right), y=\left(y_{1}, y_{2}\right)$.

$$
\begin{aligned}
(A \oplus B)\left(\left(\alpha x_{1}, \alpha x_{2}\right)+\left(y_{1}, y_{2}\right)\right. & =(A \oplus B)\left(\alpha x_{1}+y_{1}, \alpha x_{2}+y_{2}\right) \\
& =\left(A\left(\alpha x_{1}+y_{1}\right), B\left(\alpha x_{2}+y_{2}\right)\right) \\
& =\left(\alpha\left(A x_{1}, B x_{2}\right)+\left(A y_{1}, B y_{2}\right)\right) \\
& =\alpha(A \oplus B)(x)+(A \oplus B)(y) .
\end{aligned}
$$


Hence $A \oplus B$ is linear.

\section{Verification II:}

We need to show $A \in \mathbb{U}(X), B \in \mathbb{U}(Y) \Rightarrow A \oplus B \in \mathbb{U}(X \oplus Y)$. That means to show $A \oplus B$ is a unitary operator. That is, we show $\left\langle(A \oplus B)\left(x_{1}, y_{1}\right),(A \oplus\right.$ $\left.B)\left(x_{2}, y_{2}\right)\right\rangle=\left\langle\left(x_{1}, y_{1}\right),\left(x_{2}, y_{2}\right)\right\rangle$.

Consider , $\left\langle(A \oplus B)\left(x_{1}, y_{1}\right),(A \oplus B)\left(x_{2}, y_{2}\right)\right\rangle$

$$
\begin{aligned}
& =\left\langle\left(A x_{1}, B y_{1}\right),\left(A x_{2}, B y_{2}\right)\right\rangle \\
& =\left\langle A x_{1}, A x_{2}\right\rangle+\left\langle B y_{1}, B y_{2}\right\rangle
\end{aligned}
$$

(since, $X \oplus Y$ is a hilbert space, inner product can be defined like this)

$$
\begin{aligned}
& =\left\langle x_{1}, x_{2}\right\rangle+\left\langle y_{1}, y_{2}\right\rangle \\
& =\left\langle\left(x_{1}, y_{1}\right),\left(x_{2}, y_{2}\right)\right\rangle .
\end{aligned}
$$

Hence, $A \oplus B$ is a unitary operator on $X \oplus Y$.

Definition 1.2.3. If $U$ and $V$ are representations of $G$ on $X$ and $Y$, respectively, then, $U \oplus V$ defined as,

$$
(U \oplus V)(g)=U(g) \oplus V(g)
$$

is a representation of $G$, also known as Diresct sum representation.

\section{Verification I:}

We need to show that $(U \oplus V)(g)=U(g) \oplus v(g)$ is also representation of $\mathrm{G}$; where, $\mathrm{U}$ and $\mathrm{V}$ are representations of $\mathrm{G}$ on $\mathrm{X}$ and $\mathrm{Y}$ respectively. That is, to show $U \oplus V$ is a homomorphism from $\mathrm{G}$ to $\mathbb{U}(X \oplus Y)$ or to show $U \oplus V\left(g_{1} g_{2}\right)=U \oplus V\left(g_{1}\right) U \oplus V\left(g_{2}\right)$.

$$
\begin{aligned}
U \oplus V\left(g_{1} g_{2}\right)(x, y) & =\left(U\left(g_{1} g_{2}\right) \oplus V\left(g_{1} g_{2}\right)\right)(x, y) \\
& =\left(U\left(g_{1} g_{2}\right)(x), V\left(g_{1} g_{2}\right)(y)\right) \\
& =\left(U\left(g_{1}\right) U\left(g_{2}\right)(x), V\left(g_{1}\right) V\left(g_{2}\right)(y)\right) .
\end{aligned}
$$

and

$$
\begin{aligned}
U \oplus V\left(g_{1}\right) U \oplus V\left(g_{2}\right)(x, y) & =U \oplus V\left(g_{1}\right)\left(\left(U\left(g_{2}\right) \oplus V\left(g_{2}\right)(x, y)\right)\right. \\
& =U \oplus V\left(g_{1}\right)\left(U\left(g_{2}\right)(x), V\left(g_{2}\right)(y)\right) \\
& =U\left(g_{1}\right) \oplus V\left(g_{1}\right)\left(U\left(g_{2}\right)(x), V\left(g_{2}\right)(y)\right) \\
& =\left(U\left(g_{1}\right) U\left(g_{2}\right) x, V\left(g_{1}\right) V\left(g_{2}\right) y\right) \\
& =U \oplus V\left(g_{1} g_{2}\right)(x, y) ; \forall(x, y) \in X \oplus Y .
\end{aligned}
$$


Hence, $U \oplus V\left(g_{1} g_{2}\right)=U \oplus V\left(g_{1}\right) U \oplus V\left(g_{2}\right) ; \forall g_{1}, g_{2} \in \mathrm{G}$. Therefore $U \oplus V$ is a homomorphism. This shows that it is a representation of $\mathrm{G}$ on $X \oplus Y$.

Definition 1.2.4. Let $U$ be a representation of $G$ on $X$. A subspace $Y \subset X$ is called invariant if $\forall g \in G$ and $y \in Y, U(g) y \in Y$.

Theorem 1.2.5. If $Y$ is an invariant subspace, so is

$$
Y^{\perp}=\{x \in X \mid\langle x, y\rangle=0, \forall y \in Y\} .
$$

$U \uparrow Y$ and $U \uparrow Y^{\perp}$ define representations $U_{1}$ and $U_{2}$ and $U$ is equivalent to $U_{1} \oplus U_{2}$.

Conversely, $(y, 0)$ is an invariant subspace for any direct sum representation $U_{1} \oplus U_{2}$.

Proof. First, note that, $U: G \rightarrow \mathbb{U}(X)$ is a representation of $\mathrm{G}$ on Hilbert space X.

Let $x \in Y^{\perp}, y \in Y, g \in G$. Then,

$$
\begin{aligned}
\langle U(g) x, y\rangle & =\left\langle x, U^{*}(g)(y)\right\rangle \\
& =\left\langle x, U\left(g^{-1}\right) y\right\rangle \\
& =0
\end{aligned}
$$

since, $U\left(g^{-1}\right) y \in Y$. Thus, $U(g) x \in Y^{\perp}$ so, $Y^{\perp}$ is invariant. This implies that in $Y \oplus Y^{\perp}$ block decomposition of X,

$\mathrm{U}(\mathrm{g})=\left(\begin{array}{cc}U_{1}(g) & 0 \\ 0 & U_{2}(g)\end{array}\right)$ That means, $U \cong U_{1} \oplus U_{2}$.

Conversely, let $U=U_{1} \oplus U_{2}$. Then $\forall g \in \mathrm{G}$,

$$
\begin{aligned}
U(g)((y, 0)) & =\left(U_{1} \oplus U_{2}\right)(y, 0) \\
& =\left(U_{1}(g) \oplus U_{2}(g)\right)((y, 0)) \\
& =\left(U_{1}(g)(y), U_{2}(g)(0)\right) \\
& =\left(y_{1}, 0\right) \in(y, 0) .
\end{aligned}
$$

Hence $(y, 0)$ is an invariant subspace. 
Note 1.2.6. In general, $Y \subset X$ (invariant), may not have a complementary subspace that is invariant, if $U$ is not unitary and $G$ is infinite (if $G$ is finite, $U$ is equivalent to unitary).

Example 1.2.7. The non unitary representation of $\mathbb{R}$,

$$
U(x)=\left(\begin{array}{ll}
1 & x \\
0 & 1
\end{array}\right)
$$

has an invariant subspace $\left\{\left(\begin{array}{l}\alpha \\ 0\end{array}\right)\right\}$ but no complementary subspace since, say if $U: \mathbb{R} \rightarrow G L\left(\mathbb{R}^{2}\right)$ then given $U(x)$ is not unitary representation as,

$\left(\begin{array}{ll}1 & x \\ 0 & 1\end{array}\right)\left(\begin{array}{ll}1 & 0 \\ x & 1\end{array}\right)=\left(\begin{array}{cc}1+x^{2} & x \\ x & 1\end{array}\right)$

$\neq\left(\begin{array}{ll}1 & 0 \\ 0 & 1\end{array}\right)$.

Hence, $U$ is not unitary. And, $\left\{\left(\begin{array}{l}\alpha \\ 0\end{array}\right)\right\}$ is an invariant subspace since, $\forall x \in \mathbb{R}$,

$U(x)\left(\begin{array}{l}\alpha \\ 0\end{array}\right)=\left(\begin{array}{ll}1 & x \\ 0 & 1\end{array}\right)\left(\begin{array}{l}\alpha \\ 0\end{array}\right)=\left(\begin{array}{l}\alpha \\ 0\end{array}\right)$

Now, let it has a complementary subspace say, $\left(\begin{array}{l}0 \\ \beta\end{array}\right)$

then, $U(x)\left(\begin{array}{l}0 \\ \beta\end{array}\right)=\left(\begin{array}{ll}1 & x \\ 0 & 1\end{array}\right)\left(\begin{array}{l}0 \\ \beta\end{array}\right)=\left(\begin{array}{c}x \beta \\ \beta\end{array}\right)=\beta\left(\begin{array}{l}x \\ 1\end{array}\right) \notin\left\{\left(\begin{array}{l}0 \\ \beta\end{array}\right): \beta \in Y^{\perp}\right\}$.

This shows that, $(0, \beta)$ is not invariant.

Definition 1.2.8. A unitary representation $U$ of $G$ on $X$ is called irreducible if and only if the only invariant subspaces of $U$ are $\{0\}$ and $X$.

The theorem proved earlier implies that,

Corollary 1.2.1. $U$ is an irrep (we will use this for 'irreducible representation) if and only if it cannot be written as a direct sum of non trivial (that is, not zero dimensional) representations. 
Proof. Let $\mathrm{U}$ is an irrep of $\mathrm{G}$ on $\mathrm{X}$. Then the only invariant subspaces of $\mathrm{X}$ are $\{0\}$ and $X$. That means there does not exist any proper subspace of $X$ invariant under $\mathrm{U}$. Hence $\mathrm{U}$ cannot be written as direct sum of non trivial representations.

Conversely, let U cannot be written as direct sum of non trivial representations. Then we need to show that $\mathrm{U}$ is an irreducible representation.

Let if possible, $\mathrm{U}$ is a reducible representation. Then there exist $U_{1}, U_{2}$ corresponding to invariant subspaces $\mathrm{Y}$ and $\mathrm{Y}^{\prime}$ of $\mathrm{X}$ such that $U_{1}$ and $U_{2}$ are representations of $\mathrm{G}$ on $\mathrm{Y}$ and $\mathrm{Y}$ ' respectively and hence $U \cong U_{1} \oplus U_{2}$ where, $U_{1}$ and $U_{2}$ are non trivial representations which is a contradiction. Hence $\mathrm{U}$ is irrep of $\mathrm{G}$ on $\mathrm{X}$.

Example 1.2.9. Orthogonal group $O(n)$ of orthogonal matrices under multiplication has an irrep on $\mathbb{R}^{n}$.

Example 1.2.10. The representation $\phi: S_{3} \rightarrow G L_{2} \mathbb{C}$ is irreducible.

Proof. We know that $\operatorname{dim}\left(\mathbb{C}^{2}\right)=2$. Suppose it is not irreducible. Then, there exists a non zero proper invariant subspace. Now, any non zero proper $S_{3}$ invariant subspace is one dimensional. Let $\mathrm{v} \in \mathrm{W}$ then, $W=\mathbb{C} v$. Let $\sigma \in S_{3}$ then, $\phi \sigma(v)=\lambda v$ for some $\lambda \in \mathbb{C}$.

So, v must be an eigen vector for all $\phi \sigma$ with $\sigma \in S_{3}$.

Claim: $\phi(12)$ and $\phi(123)$ do not have a commom eigen vector.

On computing, we get $\phi(12)$ has eigen values 1 and -1 , with $V_{-1}=\mathbb{C} e_{1}$ and $V_{1}=\mathbb{C}\left(\begin{array}{c}-1 \\ 2\end{array}\right)$. Now, $e_{1}$ is not an eigen vector of $\phi(123)$ as $\phi\left(\begin{array}{lll}1 & 2 & 3\end{array}\right)\left(\begin{array}{l}1 \\ 0\end{array}\right)=\left(\begin{array}{c}-1 \\ 1\end{array}\right)$. Also, $\phi\left(\begin{array}{lll}1 & 2 & 3\end{array}\right)\left(\begin{array}{c}-1 \\ 2\end{array}\right)=\left(\begin{array}{l}-1 \\ -1\end{array}\right)$

So, $(-1,2)$ is not an eigen vector of $\phi(123)$. This implies, $\phi(12)$ and $\phi(123)$ have no eigen vector in common. Hence $\phi$ is irreducible.

Theorem 1.2.11. Any representation can be written as a direct sum of irreps.

Proof. We prove this by induction on $\operatorname{deg}(\mathrm{U})$. We know that $\operatorname{deg}(\mathrm{U})=\operatorname{dim}(\mathrm{X})$. If $\operatorname{deg}(\mathrm{U})=1, \mathrm{U}$ is irrep (since , any 1-dimensional representation is irreducible).

Let the result is true $\forall$ representations of $d e g<d$. Let $\operatorname{deg}(\mathrm{U})=\mathrm{d}$. If $\mathrm{U}$ is irreducible, we are done. If not, 
$U=U_{1} \oplus U_{2}$, where, $\operatorname{deg}\left(U_{1}\right)<d, \operatorname{deg}\left(U_{2}\right)<d$. Thus by induction, each $U_{i}$ is direct sum of irreducible representations. That is, $\mathrm{U}$ is direct sum of irreps. Therefore any $\mathrm{U}$ can be written as $U_{1} \oplus U_{2} \oplus \ldots \oplus U_{k}$ where $U_{i}$ is irrep $\forall \mathrm{i}=1,2, \ldots \mathrm{k}$.

Definition 1.2.12. A representation $\phi: G \rightarrow G L(V)$ is said to be completely reducible if $V=V_{1} \oplus V_{2} \oplus \ldots \oplus V_{n}$ where, $V_{i}$ are $G$-invariant subspaces and $\left.\phi\right|_{V_{i}}$ is irreducible for all $i=1,2 . . n$.

It is equivalent to saying that $\phi$ is completely reducible if $\phi \sim \phi^{1} \oplus \phi^{2} \oplus$ $\ldots \oplus \phi^{n}$ where $\phi^{i}$ are irreducible representations.

Definition 1.2.13. A representation $\phi$ is decomposable if $V=V_{1} \oplus V_{2}$ where $V_{1}, V_{2}$ are non zero G-invariant subspaces. Otherwise, $V$ is called indecomposable.

Definition 1.2.14. $\hat{G}$, known as the dual object, is the set of equivalence classes of irreps, each class consisting of unitarily equivalent irreps.

An explicit matrix realization of each $\alpha \in \hat{G}$, is $D_{i j}^{(\alpha)}(g)$ that is a $d(\alpha) \times d(\alpha)$ matrix, where $d(\alpha)$ is the degree of irrep in $\alpha$.

\subsection{The Group Algebra and the Regular Rep- resentations}

Let $\mathrm{G}$ be a finite group. Consider, $S=\left\{\sum_{g \in G} a_{g} \delta_{g} ; \delta_{g}\right.$ is a symbol, $\left.a_{g} \in \mathbb{C}\right\}$. Then, $\mathrm{S}$ is a complex vector space of dimension $\mathrm{O}(\mathrm{G})$ (i.e., order of group).

\section{Verification :}

We neeed to show that $\mathrm{S}$ is a vector space. Let $\mathrm{x}, \mathrm{y} \in S$. Then $x=\sum_{g \in G} a_{g} \delta_{g}$ and $y=\sum_{g \in G} b_{g} \delta_{g}$

1) $x+y=\sum_{g \in G} a_{g} \delta_{g}+\sum_{g \in G} b_{g} \delta_{g}=\sum_{g \in G}\left(a_{g}+b_{g}\right) \delta_{g}$;

where, $a_{g}+b_{g} \in \mathbb{C}$ (since $\mathbb{C}$ is a vector space).

This implies,$\sum_{g \in G}\left(a_{g}+b_{g}\right) \delta_{g} \in S$.

2) $\alpha x=\alpha \sum_{g \in G} a_{g} \delta_{g}=\sum_{g \in G} \alpha a_{g} \delta_{g} \in S$. since, $\alpha a_{g} \in \mathbb{C}$ and $\mathbb{C}$ is a vector space. 
So, $\alpha x \in S$. Hence, $\mathrm{S}$ is a vector space.

Now, since, $a_{g} \in \mathbb{C}$ and $\operatorname{dim}(\mathrm{S})=\mathrm{O}(\mathrm{G})$, (since, summation is over $g \in$ $\mathrm{G}) \mathrm{S}$ is a complex vector space of $\operatorname{dim}=\mathrm{O}(\mathrm{G})$.

Note 1.3.1. We consider the sequence $\left\{a_{g}\right\}_{g \in G}$ as a function so we write it as $a(g)$.

Now, we define a product, inherited from the group product for the vector space $\mathrm{S}$ such that,

$$
\delta_{g} \delta_{h}=\delta_{g h}
$$

,$\forall g \in G$.

Therefore $\left(\sum_{g} a_{g} \delta_{g}\right)\left(\sum_{g} b_{h} \delta_{h}\right)=\sum_{g, h i n G} a_{g} b_{h} \delta_{g h}$.

Now, By change of variables (i.e., let $\mathrm{gh}=\mathrm{x}, \mathrm{h}=\mathrm{y} \Rightarrow g=x y^{-1}$ ) we get,

$$
=\sum_{x}\left(\sum_{y} a\left(x y^{-1}\right) b(y) \delta_{x}\right) .
$$

Definition 1.3.2. Let $G$ be a finite group. The complex vector space $\mathscr{A}(G)$ of functions on $G$ is called the group algebra when we are given the product called CONVOLUTION as follows,

$$
(a * b)(x)=\sum_{y} a\left(x y^{-1}\right) b(y) ; a, b \in \mathscr{A}
$$

where support of each function is finite and the conjugate,

$$
a^{*}(g)=\overline{\left(a\left(g^{-1}\right)\right)}
$$

Remark 1.3.3. $(a * b)^{*}=b^{*} * a^{*}$. 


\section{Verification:}

$$
\begin{aligned}
(a * b)^{*}(x) & =\overline{(a * b)\left(x^{-1}\right)} \\
& =\overline{(a * b)(h)}=\overline{\sum_{g} a\left(h g^{-1}\right) b(g)} \\
& =\overline{\sum_{y} a(y) b\left(y^{-1} h\right)} \quad\left(h g^{-1}=y \Rightarrow g=y^{-1} h\right) \\
& =\overline{\sum_{y} b\left(y^{-1} h\right) a(y)} \\
& =\sum_{y} \overline{b\left(y^{-1} h\right) a(y)} \\
& =\sum_{y} b^{*}\left(h^{-1} y\right) a^{*}\left(y^{-1}\right) \\
& =\sum_{y} b^{*}(x y) a^{*}\left(y^{-1}\right) \\
& =\sum_{y^{-1}} b^{*}\left(x y^{-1}\right) a^{*}(y) \\
& =b^{*} * a^{*} .
\end{aligned}
$$

Let $\delta_{g}$ be the function defined as,

$$
\delta_{g}(x)= \begin{cases}1 & : \mathrm{x}=g \\ 0 & : \mathrm{x} \neq g\end{cases}
$$

then, $a=\sum a_{g} \delta_{g}$.

Verification of the statement $(*)$ written above :

$$
\begin{gathered}
\delta_{g}(x)= \begin{cases}1 & : \mathrm{x}=g \\
0 & : \mathrm{x} \neq g\end{cases} \\
\delta_{h}(x)= \begin{cases}1 & : \mathrm{x}=h \\
0 & : \mathrm{x} \neq h\end{cases} \\
\delta_{g h}(x)= \begin{cases}1 & : \mathrm{x}=g h \\
0 & : \mathrm{x} \neq g h\end{cases}
\end{gathered}
$$


$\delta_{g} \delta_{h}(x)=\sum_{y} \delta_{g}\left(x y^{-1}\right) \delta_{h}(y)$

$$
= \begin{cases}1 & : x h^{-1}=g \\ 0 & : x h^{-1} \neq g\end{cases}
$$

( when, $x y^{-1}=g, \mathrm{y}=\mathrm{h}$ )

$$
\begin{aligned}
& = \begin{cases}1 & : \mathrm{x}=g h \\
0 & : \mathrm{x} \neq g h\end{cases} \\
& =\delta_{g h}(x), \forall x \in G .
\end{aligned}
$$

$\Rightarrow \delta_{g} \delta_{h}=\delta_{g h}$

There is one more property that $\delta_{g}$ has. That is,

$$
\delta_{g^{*}}=\delta_{g^{-1}}
$$

\section{Verification:}

$$
\begin{aligned}
& \delta_{g^{*}}(x)=\overline{\delta_{g}\left(x^{-1}\right)}= \begin{cases}1 & : x^{-1}=g \\
0 & : x^{-1} \neq g\end{cases} \\
&= \begin{cases}1 & : \mathrm{x}=g^{-1} \\
0 & : \mathrm{x} \neq g^{-1}\end{cases} \\
&=\delta_{g^{-1}}(x), \forall x \in G .
\end{aligned}
$$

Hence,

$$
\delta_{g^{*}}=\delta_{g^{-1}}
$$

Now, one might wonder, why did we choose such an adjoint. So, following theorem is the reason for the same. 
Theorem 1.3.4. Let $G$ be a finite group. Let $U$ be a representation of $G$ on a Hilbert space $X$.

Define for $a \in \mathscr{A}(G)$,

$$
U_{\mathscr{A}}(a)=\sum a(g) U(g)
$$

Then $U_{\mathscr{A}}$ obeys,

(i) $U_{\mathscr{A}}(a+b)=U_{\mathscr{A}}(a)+U_{\mathscr{A}}(b)$

(ii) $U_{\mathscr{A}}(a * b)=U_{\mathscr{A}}(a) U_{\mathscr{A}}(b)$

(iii) $U_{\mathscr{A}}\left(a^{*}\right)=U_{\mathscr{A}}(a)^{*}$

(iv) $U_{\mathscr{A}}\left(\delta_{e}\right)=\mathbb{I}$; where, $e$ is the identity of the group $G$.

Conversely, if $U_{\mathscr{A}}$ obeys all the above four conditions then, $\exists$ a unitary representation $U$ of $G$ obeying (1).

\section{Proof. Forward Part:}

We have to show, $U_{\mathscr{A}}$ obeys all four properties.

(i)First,

$$
\begin{aligned}
U_{\mathscr{A}}(a+b) & =\sum(a+b)(g) U(g) \\
& =\sum(a(g)+b(g)) U(g) \\
& =\sum a(g) U(g)+\sum b(g) U(g) \\
& =U_{\mathscr{A}}(a)+U_{\mathscr{A}}(b) .
\end{aligned}
$$


(ii)Second,

$$
\begin{aligned}
U_{\mathscr{A}}(a * b) & =\sum_{g}(a * b)(g) U(g) \\
& =\sum_{h}\left(\sum_{h} a\left(g h^{-1}\right) b(h)\right) U(g) \\
& =\sum_{h}\left(\sum_{g} a\left(g h^{-1}\right) U(g)\right) b(h) \\
& =\sum_{h}\left(\sum_{x} a(x) U(x h)\right) b(h) \quad\left(g h^{-1}=x \Rightarrow h=x^{-1} g\right) \\
& =\sum_{h}\left(\sum_{x} a(x) U(x)\right)(U(h) b(h)) \\
& =\sum_{h}\left(\sum_{x} a(x) U(x)\right)(b(h) U(h)) \\
& =\sum_{x} a(x) U(x) \sum_{h} b(h) U(h) \\
& =U_{\mathscr{A}}(a) U_{\mathscr{A}}(b) .
\end{aligned}
$$

(iii) Third,

$$
\begin{aligned}
U_{\mathscr{A}}\left(a^{*}\right) & =\sum a^{*}(g) U(g) \\
& =\sum \overline{a\left(g^{-1}\right)} U(g) \\
& =\sum_{g^{-1} \in G} \overline{a(g)} U\left(g^{-1}\right) \\
& =\sum \overline{a(g)} U(g)^{*} \\
& =\sum(a(g) U(g))^{*} \\
& =\left(\sum a(g) U(g)\right)^{*} \\
& =U_{\mathscr{A}}(a)^{*} .
\end{aligned}
$$


(iv)Fourth,

$$
\begin{aligned}
U_{\mathscr{A}}\left(\delta_{e}\right) & =\sum \delta_{e}(g) U(g) \\
& =U(e)(i f g=e) \\
& =\mathbb{I}(\text { since, } \mathrm{U} \text { is a homomorphism })
\end{aligned}
$$

\section{Converse Part:}

If $U_{\mathscr{A}}$ obeys all four properties then, define,

$$
U(g)=U_{\mathscr{A}}\left(\delta_{g}\right)
$$

then, (1) holds .

Now, since, $\delta_{g} * \delta_{h}=\delta_{g h}$, Property (ii) implies,

$U(g) U(h)=U_{\mathscr{A}}\left(\delta_{g}\right) U_{\mathscr{A}}\left(\delta_{h}\right)=U_{\mathscr{A}}\left(\delta_{g} * \delta_{h}\right)=U_{\mathscr{A}}\left(\delta_{g h}\right)=U(g h)$.

And , since, $\delta_{g^{*}}=\delta_{g^{-1}}$, Property (iii) implies,

$U(g)^{*}=U_{\mathscr{A}}\left(\delta_{g}\right)^{*}=U_{\mathscr{A}}\left(\delta_{g}^{*}\right)=U_{\mathscr{A}}\left(\delta_{g}^{-1}\right)=U\left(g^{-1}\right)$.

Hence, we have,

$U(g) U(g)^{*}=U(g) U\left(g^{*}\right)=U\left(g g^{*}\right)=U_{\mathscr{A}}\left(\delta_{g g^{*}}\right)=U_{\mathscr{A}}\left(\delta_{g} * \delta_{g^{*}}\right)=U_{\mathscr{A}}\left(\delta_{g} *\right.$ $\left.\delta_{g^{-1}}\right)=U_{\mathscr{A}}\left(\delta_{g g^{-1}}\right)=U_{\mathscr{A}}\left(\delta_{e}\right)=\mathbb{I}$.

And, also,

$U(g)^{*} U(g)=U\left(g^{*} g\right)=U_{\mathscr{A}}\left(\delta_{g^{*} g}\right)=U_{\mathscr{A}}\left(\delta_{g^{*}} * \delta_{g}\right)=U_{\mathscr{A}}\left(\delta_{g^{-1}} * \delta_{g}\right)=U_{\mathscr{A}}\left(\delta_{g^{-1} g}\right)=$ $U_{\mathscr{A}}\left(\delta_{e}\right)=\mathbb{I}$.

Hence $\mathrm{U}$ is unitary.

A map $U_{\mathscr{A}}$ obeying (i) to (iv) all properties, is called a ${ }^{*}$-representation of Algebra $\mathscr{A}(G)$. 
Hence, we can conclude that there is one one correspondence between representation of $\mathrm{G}$ and *-representation of $\mathscr{A}(G)$.

The obvious representation of $\mathscr{A}(G)$ is on itself by the following multiplication:

We define, $L_{\mathscr{A}}: \mathscr{A} \rightarrow \mathbb{U}(\mathscr{A}(G))$ as,

$$
L_{\mathscr{A}}(a) b=a * b .
$$

Now, we need to define an inner product on $\mathscr{A}(G)$ so that $L_{\mathscr{A}}(a)$ becomes a ${ }^{*}$-representation.

We can take usual inner product for the same, as

$$
\langle f, g\rangle=\frac{1}{O(G)} \sum_{x \in G} \overline{f(x)} g(x) .
$$

Now, we check that $L_{\mathscr{A}}$ is a ${ }^{*}$-representation .

For that, we need to prove that,

$$
\langle h, f * g\rangle=\left\langle f^{*} * h, g\right\rangle
$$

$\forall \mathrm{f}, \mathrm{g}, \mathrm{h} \in \mathscr{A}(G)$

Consider, $L: G \rightarrow \mathbb{U}(G)$. If, $(L(x) f)(y)=f\left(x^{-1} y\right)$ then, $L_{\mathscr{A}}$ is the induced map on $\mathscr{A}$,

(since, $\left(\delta_{x} * f\right)(y)=\sum_{z} \delta_{x}\left(y z^{-1}\right) f(z)=f\left(x^{-1} y\right)$ by letting $y z^{-1}=x$ ) Then, $L_{x} \delta_{y}=\delta_{x y}$.

\section{Verification:}

$L_{x} \delta_{y}(z)=\delta_{y}\left(x^{-1} z\right)=\left\{\begin{array}{ll}1 & \mathrm{z}=x^{-1} y \\ 0 & \text { otherwise }\end{array}=\delta_{x y}(z)\right.$

Hence, $L_{x} \delta_{y}=\delta_{x y}$. So, $L_{x}$ is unitary ,

\section{Verification:}

We have to show that $\left\langle L_{x} f, L_{x} g\right\rangle=\langle f, g\rangle$. 


$$
\begin{aligned}
\left\langle L_{x} f, L_{x} g\right\rangle & =\frac{1}{O(G)} \sum_{z \in G} \overline{L_{x} f(z)} L_{x} g(z) \\
& =\frac{1}{O(G)} \sum_{z \in G} \overline{f\left(x^{-1} z\right)} g\left(x^{-1} z\right) \\
& =\frac{1}{O(G)} \sum_{x y \in G} \overline{f(y)} g(y) \\
& =\frac{1}{O(G)} \sum_{y \in G} \overline{f(y)} g(y) \\
& =\langle f, g\rangle .
\end{aligned}
$$

Now, let $\mathrm{g}(\mathrm{z}) \in \mathscr{A}(G)$. Then,

$$
g(z)=\sum_{y \in G} a(y) \delta_{y}(z)
$$

for any $\mathrm{g} \in \mathscr{A}$.

Hence, we can conclude that, $\left(\sqrt{O(G)} \delta_{y}\right)_{y \in G}$ is a basis.

$L_{x}$ is unitarily equivalent to ,

$$
\langle h, f * g\rangle=\left\langle f^{*} * h, g\right\rangle .
$$

Theorem 1.3.5. $\left(L_{x} f(y)\right) \equiv f\left(x^{-1} y\right)$ on $\mathscr{A}(G)$ with inner product ,

$$
\langle f, g\rangle=\frac{1}{O(G)} \sum_{x \in G} \overline{f(x)} g(x)
$$

is a unitary representation of $G$. It is called left regular representation.

Similarly, $R_{\mathscr{A}}(f) g=g * \bar{f}^{*}$, the right multiplication, is also a representation of $\mathscr{A}$ induced by,

$$
\left(R_{x} f\right)(y)=f(y x)
$$

known as the Right regular representation.

The consequence of existence of this ' $L$ ' is the following corollary. 
Corollary 1.3.1. The functions $D_{i j}^{\alpha}$ separate points that is, for any $x, y \in$ $G$ with $x \neq y, \exists$ a function $f$ of the form:

$$
f=\sum_{k=1}^{n} C_{k i j} D_{i k j k}^{\alpha_{k}}
$$

such that, $f(x)=1, f(y)=0$

Proof. Consider, $f(z)=O(G)\left\langle\delta_{x}, L(z) \delta_{e}\right\rangle$,

then,

$$
\begin{aligned}
f(x) & =O(G)\left\langle\delta_{x}, L(x) \delta_{e}\right\rangle \\
& =O(G) \frac{1}{O(G)} \sum_{y \in G} \overline{\delta_{x}(y)} L_{x} \delta_{e}(y) \\
& =\sum_{y \in G} \overline{\delta_{x}(y)} \delta_{e}\left(x^{-1} y\right) \\
& =1 .(\text { if } \mathrm{y}=\mathrm{x}) \\
f(y) & =O(G)\left\langle\delta_{x}, L(y) \delta_{e}\right\rangle \\
& =O(G) \frac{1}{O(G)} \sum_{z \in G} \overline{\delta_{x}(z)} L(y) \delta_{e}(z) \\
& =\sum_{z \in G} \overline{\delta_{x}(z)} \delta_{e}\left(y^{-1} z\right) \\
& =0 .
\end{aligned}
$$

Now, $L: G \rightarrow \mathbb{U}(\mathscr{A}(G))$ as,

$$
z \longmapsto L_{z}
$$

where,

$$
L_{z}: \mathscr{A}(G) \rightarrow \mathscr{A}(G)
$$

We know that,

$\hat{G}=\{\alpha: \alpha$ is irreducible unitary representation of $\mathrm{G}\}$

Let $\alpha_{k} \in \hat{G}$

$$
\alpha_{k}: G \rightarrow \mathbb{U}\left(\mathbb{H}_{\alpha_{k}}\right)
$$


as,

$$
z \longmapsto \alpha_{k}(z)
$$

is a representation, where, $\alpha_{k}(z): \mathbb{H}_{\alpha_{k}} \rightarrow \mathbb{H}_{\alpha_{k}}$ and $\operatorname{dim}\left(\mathbb{H}_{\alpha_{k}}\right)=t_{\alpha_{k}}$ So, the associated matrix is,

$$
\left(D_{i j}^{\alpha_{k}}(z)\right)_{t_{\alpha_{k} \times t_{\alpha_{k}}}}
$$

therefore , $\oplus_{k=1}^{n} \alpha_{k}(z): \oplus_{k=1}^{n} \mathbb{H}_{\alpha_{k}} \rightarrow \oplus_{k=1}^{n} \mathbb{H}_{\alpha_{k}}$

Now, let

$$
\mathbb{H}=\oplus_{k=1}^{n} \mathbb{H}_{\alpha_{k}}
$$

and,

$$
\alpha(z)=\oplus_{k=1}^{n} \alpha_{k}(z)
$$

so, we get,

$$
\alpha(z): G \rightarrow \mathbb{U}(\mathbb{H})
$$

defined as,

$$
z \longmapsto \alpha(z)
$$

Now, $L: G \rightarrow \mathbb{U}(\mathscr{A}(G))$ is again a representation of $\mathrm{G}$.

But since, $\mathrm{L}$ is a representation, it is unitarily equivalent to a direct sum of irreducibles.

So, given $\mathrm{L}$ and $\alpha$ are unitarily equivalent representations of $\mathrm{G}$ on $\mathscr{A}(G)$ and $\mathbb{H}$ respectively. Therefore, there exists $U: \mathscr{A}(G) \rightarrow \mathbb{H}$ such that,

$$
U L(z) U^{-1}=\alpha(z)
$$

So, $\forall \mathrm{z} \in \mathrm{G}$,

$L(z)=U^{-1} \alpha(z) U, \forall z \in G$.

That is, $L(z)=U^{-1}\left(\oplus_{k=1}^{n} \alpha_{k}(z)\right) U, \forall z \in G$ or, $L(z)=\oplus_{k=1}^{n} U^{-1} \alpha_{k}(z) U$.

Hence $L(z)=\oplus_{k=1}^{n} U^{*} \alpha_{k}(z) U$.

Therefore, $(L(z))_{x, y}=\sum_{k, i, j} M_{x i}^{\left(\alpha_{k}\right)} D_{i j}^{\left(\alpha_{j}\right)}(z){\overline{M_{y j}}}^{\left(\alpha_{k}\right)}$

for some numbers, $M_{x i}^{\left(\alpha_{k}\right)}$.

Hence, this yields,

$$
f=\sum_{k=1}^{n} C_{k i j} D_{i k j k}^{\left(\alpha_{k}\right)}
$$


This completes the proof.

Remark 1.3.6. (1) $A{ }^{*}$-representation of $\mathscr{A}(G)$ on a Hilbert space $X$ is called irreducible iff it leaves no non trivial subspace invariant.

(2) $U_{\mathscr{A}}$ is an irreducible representation of $\mathscr{A}(G)$ iff $U$ is an irreducible representation of $G$.

\subsection{Schur's Lemma}

\section{Theorem 1.4.1. First form:}

Let $U_{\mathscr{A}}$ be an irreducible representation of $\mathscr{A}(G)$ on X.Let $A \in H o m(X)$ obeys

$$
A U_{\mathscr{A}}(f)=U_{\mathscr{A}}(f) A
$$

$\forall f \in \mathscr{A}(G)$.

(equivalently, $A U(x)=U(x) A \forall x \in G$ )

Then, $A=c \mathbb{I}$ for some constant $c$.

Proof. Replacing f by $f^{*}$ and taking adjoints, we see that ,

$$
A^{*} U_{\mathscr{A}}(f)=U_{\mathscr{A}}(f) A^{*}
$$

Thus, (1) holds if $\mathrm{A}$ is replaced by $B=\frac{1}{2}\left(A+A^{*}\right)$ or $C=\frac{1}{2 i}\left(A-A^{*}\right)$.

We note that, $A=B+i C$. So, if $\mathrm{B}, \mathrm{C}$ are constants, so is $\mathrm{A}$.

Hence, it suffices to prove the theorem with $A=A^{*}$.

Let $\lambda$ be an eigen value of $\mathrm{A}$ and let $(A-\lambda) u=0$.

Then,

$$
(A-\lambda) U_{\mathscr{A}}(f) u=U_{\mathscr{A}}(f)(A-\lambda) u=0
$$

Thus, $\{u \mid(A-\lambda) u=0\}$ is an invariant subspace.

Since, $\lambda$ is an eigen value, space is not $\{0\}$. So, it must be $\mathrm{X}$.

Therefore, $A=\lambda \mathbb{I}$ where $\lambda$ is the constant. Hence proved.

\section{Theorem 1.4.2. Second Form:}

Let $U, V$ be two irreps of a finite group $G$ on spaces $X$ and $Y$ respectively. Let $T: X \rightarrow Y$ such that,

$$
T U(x)=V(x) T
$$


$\forall x \in G$.

Then, either, $T=0$ or $U, V$ are unitarily equivalent and $T$ is unique upto constant.

Proof. Replacing $\mathrm{x}$ by $x^{-1}$ and taking adjoints to see that

$$
T^{*} V(x)=U(x) T^{*}
$$

Thus,

$$
\left(T^{*} T\right) U(x)=U(x)\left(T^{*} T\right)
$$

and,

$$
\left(T T^{*}\right) V(x)=V(x)\left(T T^{*}\right)
$$

Thus, by previous theorem, $T^{*} T=c \mathbb{I}$ and $T T^{*}=c \mathbb{I}$. Either, $\mathrm{c}=0 \Rightarrow T=0$ or else, $W=c^{-1 / 2} T$ is unitary and

$$
W U(x)=U(x) W
$$

so, $\mathrm{U}, \mathrm{V}$ are unitarily equivalent.

If they are unitarily equivalent and both $\mathrm{T}$ and $\mathrm{S}$ obey (2), then, $T S^{*}=$ $c_{1} \mathbb{I}$ by same argument. So, $T=T S^{*} S c_{s}^{-1}=c_{1} c_{s}^{-1} S$.

For general representations $\mathrm{U}$ and $\mathrm{V}$, a map $\mathrm{T}$ obeying (2), is called an intertwining map for $\mathrm{U}, \mathrm{V}$.

Corollary 1.4.1. Let $G$ be an abelian group. Then, every irrep has degree 1.

Proof. Let U be an irrep of G on X. By Schur's lemma (first form), each U(x) is a constant multiple of identity. Thus, every subspace of $\mathrm{X}$ is invariant. This is consistent with irreducible only if $\operatorname{dim}(\mathrm{X})=1$.

\subsection{Tensor Products}

Let $\mathrm{X}$ and $\mathrm{Y}$ be finite dimensional Hilbert spaces. Then the tensor product $\mathrm{X} \otimes \mathrm{Y}$ is defined as the vector space of bi-antilinear maps with a certain inner product. Let $\mathrm{B} \in \mathrm{X} \otimes \mathrm{Y}$. Then, $\mathrm{B}$ is a map from $\mathrm{X} \times \mathrm{Y} \rightarrow \mathbb{C}$ such that, 
$\mathrm{B}(\mathrm{u}, \alpha \mathrm{v}+\beta \mathrm{w})=\bar{\alpha} B(u, v)+\bar{\beta} B(u, w) \forall u \in X, v, w \in Y \alpha, \beta \in \mathbb{C}$

$\mathrm{B}(\alpha \mathrm{u}+\beta \mathrm{v}, \mathrm{w})=\bar{\alpha} B(u, w)+\bar{\beta} B(v, w) \forall u, v \in X, w \in Y \alpha, \beta \in \mathbb{C}$

Now, we show that $X \otimes Y$ is a vector space.

Let $\mathrm{A}, \mathrm{B} \in X \otimes Y$.Then, $\mathrm{A}: \mathrm{X} \times \mathrm{Y} \rightarrow \mathbb{C}$ and $\mathrm{B}: \mathrm{X} \times \mathrm{Y} \rightarrow \mathbb{C}$

$\mathrm{A}(\mathrm{u}, \alpha \mathrm{v}+\beta \mathrm{w})=\bar{\alpha} A(u, v)+\bar{\beta} A(u, w)$.

$\mathrm{A}(\alpha \mathrm{u}+\beta \mathrm{v}, \mathrm{w})=\bar{\alpha} A(u, w)+\bar{\beta} A(v, w)$.

$\mathrm{B}(\mathrm{u}, \alpha \mathrm{v}+\beta \mathrm{w})=\bar{\alpha} B(u, v)+\bar{\beta} B(u, w)$.

$\mathrm{B}(\alpha \mathrm{u}+\beta \mathrm{v}, \mathrm{w})=\bar{\alpha} B(u, w)+\bar{\beta} B(v, w)$.

Now, Consider, $\mathrm{A}+\mathrm{B}(\mathrm{u}, \alpha \mathrm{v}+\beta \mathrm{w})$.

$\mathrm{A}+\mathrm{B}(\mathrm{u}, \alpha \mathrm{v}+\beta \mathrm{w})=\mathrm{A}(\mathrm{u}, \alpha \mathrm{v}+\beta \mathrm{w})+\mathrm{B}(\mathrm{u}, \alpha \mathrm{v}+\beta \mathrm{w})=\bar{\alpha} A(u, v)+\bar{\beta} A(u, w)+\bar{\alpha} B(u, v)+$ $\bar{\beta} \mathrm{B}(\mathrm{u}, \mathrm{w})=\bar{\alpha}(\mathrm{A}(\mathrm{u}, \mathrm{v})+\mathrm{B}(\mathrm{u}, \mathrm{v}))+\bar{\beta}(\mathrm{A}(\mathrm{u}, \mathrm{w})+\mathrm{B}(\mathrm{u}, \mathrm{w}))=\bar{\alpha}(\mathrm{A}+\mathrm{B})(\mathrm{u}, \mathrm{v})+\bar{\beta}(\mathrm{A}+\mathrm{B})(\mathrm{u}, \mathrm{w})$.

Hence $\mathrm{A}+\mathrm{B} \in \mathrm{X} \otimes \mathrm{Y}$.

Now, consider,

$\mathrm{aA}(\mathrm{u}, \alpha \mathrm{v}+\beta \mathrm{w})=\mathrm{a}(\bar{\alpha} \mathrm{A}(\mathrm{u}, \mathrm{v})+\bar{\beta} \mathrm{A}(\mathrm{u}, \mathrm{w}))=\bar{\alpha} \mathrm{aA}(\mathrm{u}, \mathrm{v})+\bar{\beta} \mathrm{aA}(\mathrm{u}, \mathrm{w})$.

Hence $\mathrm{aA} \in \mathrm{X} \otimes \mathrm{Y}$. So, $\mathrm{X} \otimes \mathrm{Y}$ is a vector space.

Let $\mathrm{u} \in \mathrm{X}, \mathrm{v} \in \mathrm{Y}$.Then, define $\mathrm{u} \otimes \mathrm{v} \in \mathrm{X} \otimes \mathrm{Y}$ by

$$
(u \otimes v)(w, z)=\langle w, u\rangle\langle z, v\rangle .
$$

Then, $(\mathrm{u}, \mathrm{v}) \longmapsto(\mathrm{u} \otimes \mathrm{v})$ is a bilinear map of $\mathrm{X} \times \mathrm{Y}$ into $\mathrm{X} \otimes \mathrm{Y}$. Also, $\mathrm{u} \otimes \mathrm{v}$ spans $\mathrm{X} \otimes \mathrm{Y}$.

Note 1.5.1. Loosely speaking, a bilinear map satisfies:

$B(x+y, z)=B(x, z)+B(y, z)$ that is, additivity in first coordinate.

$B(x, y+z)=B(x, y)+B(x, z)$ that is, additivity in second coordinate.

$B(c x, y)=c B(x, y)=B(x, c y)$ that is, preserves scalar multiplication in each coordinate.

Example 1.5.2. Examples of bilinear maps:

1. Matrix multiplication is a bilinear map:

$$
M(m, n) \times M(n, p) \rightarrow M(m, p) .
$$


2. Let $V$ be a vector space over $\mathbb{R}$ carrying an inner product. Then the inner product is a bilinear map $V \times V \rightarrow \mathbb{F}$.

Now, getting back to the tensor products, if we have two finite dimensional Hilbert spaces $\mathrm{X}$ and $\mathrm{Y}$ and given that $\left\{e_{i}\right\}_{i=1}^{n}$ is a basis for $\mathrm{X}$ and $\left\{f_{j}\right\}_{j=1}^{m}$ is a basis for $\mathrm{Y}$, then $\left\{e_{i} \otimes f_{j}\right\} i=1, j=1^{n, m}$ is a basis for $\mathrm{X} \otimes \mathrm{Y}$.

To prove this, we just need to show that $\left\{e_{i} \otimes f_{j}\right\} i=1, j=1^{n, m}$ is linearly independent since we already know that it spans the space $\mathrm{X} \otimes \mathrm{Y}$.So, let us consider,

$$
\sum_{i, j} \alpha_{i, j}\left(e_{i} \otimes f_{j}\right)=0
$$

This implies that,

$$
\sum_{i, j} \alpha_{i, j}\left(e_{i} \otimes f_{j}\right)(x, y)=0, \forall x \in X, y \in Y
$$

i.e.,

$$
\sum_{i, j} \alpha_{i, j}\left\langle x, e_{i}\right\rangle\left\langle y, f_{j}\right\rangle=0
$$

We need to show that $\alpha_{i, j}=0, \forall i, j$.Suppose this is not true. Then, choose $x \in \mathrm{X}$ and $y \in \mathrm{Y}$ such that $\left\langle x, e_{i}\right\rangle \neq 0$ if $x \in\left(\operatorname{span}\left\{e_{j}\right\}\right)^{\perp}$ where $j \neq i$ and, $\left\langle y, f_{i}\right\rangle \neq 0$ if $\mathrm{y} \in\left(\operatorname{span}\left\{f_{j}\right\}\right)^{\perp}$ where $j \neq i$. Then, for this choice of $\mathrm{x}$ and $\mathrm{y}$,

$$
\sum_{i, j} \alpha_{i, j}\left\langle x, e_{i}\right\rangle\left\langle y, f_{j}\right\rangle \neq 0
$$

which is a contradiction.

Hence, $\alpha_{i, j}=0$. Thus, the set is linearly independent.Therefore, $\left\{e_{i} \otimes f_{j}\right\} i=1, j=1^{n, m}$ is a basis for $\mathrm{X} \otimes \mathrm{Y}$.

So, now we can conclude that, $\operatorname{dim}(\mathrm{X} \otimes \mathrm{Y})=\operatorname{dim}(\mathrm{X}) \operatorname{dim}(\mathrm{Y})$. We can give a unique inner product to $\mathrm{X} \otimes \mathrm{Y}$ in which,

$$
\langle u \otimes Y, B\rangle=B(u, v)
$$

where $\mathrm{B} \in X \otimes Y$. This inner product is such that if $\left\{e_{i}\right\}$ and $\left\{f_{j}\right\}$ are orthonormal bases for $\mathrm{X}$ and $\mathrm{Y}$ respectively then, $e_{i} \otimes f_{j}$ is an orthonormal basis for $\mathrm{X} \otimes \mathrm{Y}$.

Now, suppose that $\mathrm{A} \in \operatorname{Hom}(\mathrm{X})$ and $\mathrm{B} \in \operatorname{Hom}(\mathrm{Y})$ then, $\mathrm{A} \otimes \mathrm{B} \in \operatorname{Hom}(\mathrm{X}$ $\otimes \mathrm{Y})$ is defined as

$$
(A \otimes B)(C)(u, v)=C(A * u, B * v)
$$


where $\mathrm{C} \in \mathrm{X} \otimes \mathrm{Y}, \mathrm{u} \in \mathrm{X}, \mathrm{v} \in \mathrm{Y}$ so that,

$$
(A \otimes B)(u \otimes v)=A u \otimes B v .
$$

Now, we move further to define what is called the Tensor Product Representation. Given $\mathrm{W}$ and $\mathrm{V}$ to be two representations of a group $\mathrm{G}$ on spaces $\mathrm{X}$ and $\mathrm{Y}$ respectively, we define $\mathrm{W} \otimes \mathrm{V}$ to be a representation of $\mathrm{G}$ on $\mathrm{X} \otimes \mathrm{Y}$ by,

$$
(W \otimes V)(x)=W(x) \otimes V(x)
$$

known as the tensor product representation. In general, if $W^{\alpha}$ and $V^{\beta}$ are irreps, $W^{\alpha} \otimes V^{\beta}$ is not irrep but a direct sum of irreducibles $\bigoplus_{\gamma \in \hat{G}} n_{\alpha \beta}^{\gamma} U^{(\gamma)}$ given for $\alpha, \beta \in \hat{G}$ that is,

$$
W^{\alpha} \otimes V^{\beta} \cong \bigoplus_{\gamma \in \hat{G}} n_{\alpha \beta}^{\gamma} U^{(\gamma)} .
$$

Here, $n_{\alpha \beta}^{\gamma}$ are called Clebsch-Gordan integers representing the noumber of times $U^{(\gamma)}$ appears in the direct sum.

Moreover,

$$
D_{i j}^{\alpha}(x) D_{k l}^{\beta}(x)=\sum_{m, p, q} C_{i j ; k l ; p q}^{\alpha \beta m} D_{p q}^{\gamma_{m}}(x)
$$

where $D_{i j}$ is the matrix realization for the irreducible representations, $C_{i j ; k l ; p q}^{\alpha \beta m}$ are the constants and sum is over $\mathrm{m}=1,2, \ldots \mathrm{M}$ and for each $\mathrm{m}$, there is associated some $\gamma_{m} \in \hat{G}$ and $\mathrm{p}, \mathrm{q}=1,2, \ldots, d_{\gamma_{m}}$. 


\section{Chapter 2}

\section{Orthogonality Relations}

\subsection{Morphisms}

Definition 2.1.1. Let $\phi: G \rightarrow G L(V)$ and $\psi: G \rightarrow G L(W)$ be representations. A morphism from $\phi$ to $\psi$ is a linear map $T: V \rightarrow W$ such that $T \phi_{g}=\psi_{g} T, \forall g \in G$.

The set of all morphisms from $\phi$ to $\psi$ is denoted by $\operatorname{Hom}_{G}(\phi, \psi)$ and $\operatorname{Hom}_{G}(\phi, \psi) \subset \operatorname{Hom}(V, W)$.

Remark 2.1.2. 1. If $T \in \operatorname{Hom}_{G}(\phi, \psi)$ is invertible, then $\phi \equiv \psi$ and $T$ is isomorphism.

2. $T: V \rightarrow V$ belongs to $\operatorname{Hom}_{G}(\phi, \phi)$ iff $T \phi_{g}=\phi_{g} T, \forall g \in G$ that is, $T$ commutes with $\phi(g)$. Therefore, in particular, $I: V \rightarrow V$ is always an element of $\mathrm{Hom}_{G}(\phi, \phi)$.

\subsection{The Orthogonality Relations}

Let $\mathrm{G}$ be a finite group. Consider $\phi: G \rightarrow G L_{n}(\mathbb{C})$ to be a representation. Let $\phi_{g}=\left(\phi_{i j}(g)\right)$ where $\left(\phi_{i j}(g)\right) \in \mathbb{C}, \forall 1 \leq i, j \leq n$. That is, there exist $n^{2}$ functions $\phi_{i j}: G \rightarrow \mathbb{C}$ assosciated to degree $\mathrm{n}$ representation $\phi$. Whenever $\phi$ is irreducible and unitary, then functions of the form $\phi_{i j}: G \rightarrow \mathbb{C}$ form an orthogonal basis for $\mathbb{C}^{G}$. 
Proposition 2.2.1. Suppose we have two representations $\phi: G \rightarrow G L(V)$ and $\psi: G \rightarrow G L(W)$ and $T: V \rightarrow W$ is a linear transformation. Then, the following statements hold:

(a). $T^{\#}=\frac{1}{O(G)} \sum_{g \in G} \psi_{g^{-1}} T \phi_{g}$ belongs to $\operatorname{Hom}_{G}(\phi, \psi)$.

(b). If $T \in \operatorname{Hom}_{G}(\phi, \psi)$ then, $T^{\#}=T$.

(c). $P: \operatorname{Hom}(V, W) \rightarrow \operatorname{Hom}(\phi, \psi)$ defined by $P(T)=T^{\#}$ is an onto linear map.

Proof. To prove statement (a), consider,

$$
\begin{aligned}
T^{\#} \phi_{h} & =\frac{1}{O(G)} \sum_{g \in G} \psi_{g^{-1}} T \phi_{g} \phi_{h} \\
& =\frac{1}{O(G)} \sum_{g \in G} \psi_{g^{-1}} T \phi_{g h} .
\end{aligned}
$$

Suppose $\mathrm{gh}=\mathrm{x}$. Then, $g^{-1}=h x^{-1}$. Therefore, our expression becomes,

$$
\begin{aligned}
T^{\#} \phi_{h} & =\frac{1}{O(G)} \sum_{x \in G} \psi_{h x^{-1}} T \phi_{x} \\
& =\psi_{h} \frac{1}{O(G)} \sum \psi_{x^{-1}} T \phi_{x} \\
& =\psi_{h} T^{\#} .
\end{aligned}
$$

Hence, $T^{\#} \in \operatorname{Hom}_{G}(\phi, \psi)$.

Next, to prove (b), if $T \in \operatorname{Hom}_{G}(\phi, \psi)$, then

$$
\begin{aligned}
T^{\#} & =\frac{1}{O(G)} \sum_{g \in G} \psi_{g^{-1}} T \phi_{g} \\
& =\frac{1}{O(G)} \sum_{g \in G} \psi_{g^{-1}} \psi_{g} T \\
& =\frac{1}{O(G)} \sum_{g \in G} T=\frac{1}{O(G)} O(G) T \\
& =T
\end{aligned}
$$


Now, we prove (c),

Consider,

$$
\begin{aligned}
P: \operatorname{Hom}(V, W) \rightarrow & \operatorname{Hom}_{G}(\phi, \psi) \text { defined as } \\
& P\left(c_{1} T_{1}+c_{2} T_{2}\right)=\left(c_{1} T_{1}+c_{2} T_{2}\right)^{\#} \\
\left(c_{1} T_{1}+c_{2} T_{2}\right)^{\#}= & \frac{1}{O(G)} \sum_{g \in G} \psi_{g^{-1}}\left(c_{1} T_{1}+c_{2} T_{2}\right) \phi_{g} \\
= & c_{1} \frac{1}{O(G)} \sum_{g \in G} \psi_{g^{-1}} T_{1} \phi_{g}+c_{2} \frac{1}{O(G)} \sum_{g \in G} \psi_{g^{-1}} T_{2} \phi_{g} \\
= & c_{1} T^{\#}+c_{2} T_{2}^{\#} \\
= & c_{1} P\left(T_{1}\right)+c_{2} P\left(T_{2}\right) .
\end{aligned}
$$

Hence, the map $\mathrm{P}$ is linear. Now, we know from (b) that, if $T \in \operatorname{Hom}_{G}(\phi, \psi), T^{\#}=$ $P(T)=T$. Thus, it is onto .

Before moving further, let us restate the Schur's Lemma:

Let $\phi$ and $\psi$ be irreps of $\mathrm{G}$ and $T \in \operatorname{Hom}_{G}(\phi, \psi)$. Then either $\mathrm{T}$ is invertible or $\mathrm{T}=0$. Consequently,

(a). If $\phi \nsim \psi$ then, $\operatorname{Hom}_{G}(\phi, \psi)=0$.

(b). If $\phi=\psi$ then, $T=\lambda I$, where $\lambda \in \mathbb{C}$.

Proposition 2.2.2. Let $\phi: G \rightarrow G L(V)$ and $\psi: G \rightarrow G L(W)$ be irreps of $G$ and let $T: V \rightarrow W$ be linear. Then,

(a). If $\phi \not \psi$ then, $T^{\#}=0$.

(b). If $\phi \sim \psi$ then, $T^{\#}=\frac{\operatorname{Trace}(T) I}{\operatorname{deg} \phi}$.

Proof. Let $\phi \nsim \psi$. Then, $\operatorname{Hom}_{G}(\phi, \psi)=0$ by Schur's lemma since if that is not the case then there exists $0 \neq T \in \operatorname{Hom}_{G}(\phi, \psi)$ invertible and hence $\phi \sim \psi$ that is a contradiction.So, $T^{\#}=0$ since $T^{\#} \in \operatorname{Hom}_{G}(\phi, \psi)$. Next,we suppose $\phi=\psi$. Then, by Schur's lemma, $T^{\#}=\lambda I$ for some $\lambda \in \mathbb{C}$. Now, as $T^{\#}: V \rightarrow V($ since, $\phi=\psi)$ we have, $\operatorname{Trace}\left(T^{\#}\right)=\operatorname{Trace}(\lambda I)=\lambda \operatorname{Trace}(I)=$ $\lambda \operatorname{dim} V=\lambda \operatorname{deg} \phi$. Hence, $\lambda=\frac{\operatorname{Trace}(\lambda I)}{\operatorname{deg} \phi}=\frac{\operatorname{Trace}\left(T^{\#}\right)}{\operatorname{deg} \phi}$. Now, we compute the trace.Note that, 
(We use 'Tr' for Trace for convenience).

$\operatorname{Tr}\left(T^{\#}\right)=\frac{1}{O(G)} \sum_{g \in G} \operatorname{Tr}\left(\phi_{g^{-1}} T \phi_{g}\right)=\frac{1}{O(G)} \sum_{g \in G} \operatorname{Tr}(T)=\frac{O(G)}{O(G)} \operatorname{Tr}(T)=\operatorname{Tr}(T)$.

Thus, $\lambda=\frac{\operatorname{Tr}(T) I}{\operatorname{deg} \phi}$. Hence proved.

Suppose we have two representations $\phi: G \rightarrow G L_{n}(\mathbb{C})$ and $\psi: G \rightarrow$ $G L_{m}(\mathbb{C})$. Then, $\operatorname{Hom}(V, W)=M_{m n}(\mathbb{C}) . H_{\operatorname{Hom}}(\phi, \psi) \subseteq M_{m n}(\mathbb{C})$. Andthus, $P$ : $\operatorname{Hom}(V, W) \rightarrow \operatorname{Hom}_{G}(\phi, \psi)$ defined as $P(T)=T^{\#}$ can be viewed as a linear transformation $P: M_{m n}(\mathbb{C}) \rightarrow M_{m n}(\mathbb{C})$.Note that, the standard basis for $M_{m n}(\mathbb{C})$ is $\left\{E_{11}, E 12, \ldots, E_{m n}\right\}$, where $E_{i j}$ is the $m \times n$ matrix with 1 at ij position and 0 otherwise. Therefore, $a_{i j}=\sum_{i, j} a_{i j} E i j$.

Lemma 2.2.3. Let $A \in M_{r m}(\mathbb{C})$ and $B \in M_{n s}(\mathbb{C})$ and $E_{k i} \in M_{m n}(\mathbb{C})$. Then, $\left(A E_{k i} B\right)_{l j}=a_{l k} b_{i j}$ holds, where $A=\left(a_{i j}\right)$ and $B=\left(b_{i j}\right)$.

Proof.

$$
\left(A E_{k i} B\right)_{l j}=\sum\left(a_{l x}\left(E_{k i}\right)_{x y} b_{y j}\right)=a_{l k} b_{i j}
$$

since, all terms in the above sum are zero except when $\mathrm{x}=\mathrm{k}, \mathrm{y}=\mathrm{i}$.

Lemma 2.2.4. Let $\phi: G \rightarrow U_{n}(\mathbb{C})$ and $\psi: G \rightarrow U_{m}(\mathbb{C})$ be two unitary representations. Let $A=E_{k i} \in M_{m n}(\mathbb{C})$. Then, $A_{l j}^{\#}=\left\langle\phi_{i j}, \psi_{k l}\right\rangle$.

Proof. Since, $\psi$ is unitary, $\psi_{g^{-1}}=\psi_{g}^{-1}=\psi_{g}^{*}$. This implies, $\psi_{l k}\left(g^{-1}\right)=\overline{\psi_{k l}(g)}$.

$$
A_{l j}^{\#}=\frac{1}{O(G)} \sum_{g \in G}\left(\phi_{g^{-1}} E_{k i} \phi_{g}\right)_{l j}=\frac{1}{O(G)} \sum_{g \in G} \psi_{l k}\left(g^{-1}\right) \phi_{i j}(g)
$$

(by lemma done before.) Therefore,

$$
A_{l j}^{\#}=\frac{1}{O(G)} \sum_{g \in G} \overline{\psi_{k l}(g)} \phi_{i j}(g)=\left\langle\phi_{i j}, \psi_{k l}\right\rangle
$$

Hence proved. 


\subsection{Schur Orthogonality Relations}

Theorem 2.3.1. Let $\phi: G \rightarrow U_{n}(\mathbb{C})$ and $\psi: G \rightarrow U_{m}(\mathbb{C})$ be inequivalent irreducible unitary representations. Then, (a). $\left\langle\phi_{i j}, \psi_{k l}\right\rangle=0$.

(b). $\left\langle\phi_{i j}, \phi_{k l}\right\rangle=\left\{\begin{array}{ll}1 / n & ; i=k, j=l \\ 0 & ; \text { otherwise. }\end{array}\right.$.

Proof. To prove first part, note that if $A=E_{k i} \in M_{m n}(\mathbb{C})$ then, $A_{l j}^{\#}=$ $\left\langle\phi_{i j}, \psi_{k l}\right\rangle=0$ since representations are given to be inequivalent. (We can conclude this using previous proposition and lemma). Now, if $\phi=\psi$ then again by same proposition and lemma, for $A=E_{k i} \in M_{n}(\mathbb{C})($ since, $\mathrm{m}=\mathrm{n})$,

$$
A^{\#}=\frac{\operatorname{Tr}\left(E_{k i}\right) I}{n}
$$

and

$$
A_{l j}^{\#}=\left\langle\phi_{i j}, \phi_{k l}\right\rangle
$$

First, $j \neq l$, then since $I_{l j}=0$, this gives $A_{l j}^{\#}=0$ and hence, $\left\langle\phi_{i j}, \phi_{k l}\right\rangle=0$. Now, let $i \neq k$, then $E_{k i}$ has only zeros on diagonal. This gives $\operatorname{Tr}\left(E_{k i}\right)=0$. Therefore, again, $0=A_{l j}^{\#}=\left\langle\phi_{i j}, \phi_{k l}\right\rangle$. Hence, if we consider the case when $\mathrm{i}=\mathrm{k}$ and $\mathrm{l}=\mathrm{j}$ then $E_{k i}$ has a single 1 on diagonal and all other elements as zero.Thus, $\operatorname{Tr}\left(E_{k i}\right)=1$ and so, $\frac{1}{n}=A_{l j}^{\#}=\left\langle\phi_{i j}, \phi_{k l}\right\rangle$.

Hence, proved.

Corollary 2.3.1. For an irreducible unitary representation $\phi$ of degree $d$, the $d^{2}$ functions,

$$
\left\{\sqrt{d} \phi_{i j} \mid 1 \leq i, j \leq d\right\}
$$

form an orthonormal set.

Hence, according to above theorem, the entries of inequivalent unitary representations of $\mathrm{G}$ form an orthogonal set of non zero vectors in $\mathscr{A}(G)$, the group algebra.Therefore, $\mathrm{G}$ has atmost $\mathrm{O}(\mathrm{G})$ equivalence classes of irreps - Now, if $\phi^{(1)}, \ldots, \phi^{(s)}$ are a complete set of representatives of equivalence classes of irreps of $\mathrm{G}$ and $d_{i}=\operatorname{deg} \phi^{(i)}$ then, $d_{1}^{2}+d_{2}^{2}+\ldots .+d_{s}^{2}$ functions $\left\{\sqrt{d}_{k} \phi_{i j}^{(k)} \mid 1 \leq i, j \leq d_{k}\right\}$ form an orthonormal set of vectors in $\mathscr{A}(G)$ and hence, $s \leq d_{1}^{2}+\ldots+d_{s}^{2} \leq O(G)$ since $d_{i} \geq 1, \forall i$. 


\subsection{Characters and Class functions}

Definition 2.4.1. Let $\phi: G \rightarrow G L(V)$ be a representation. The character $\chi_{\phi}: G \rightarrow \mathbb{C}$ of $\phi$ is defined as $\chi_{\phi}(g)=\operatorname{Tr}\left(\phi_{g}\right)$. We call the character of an irrep as irreducible character. Suppose we have a representation $\phi: G \rightarrow$ $G L_{n}(\mathbb{C})$ given by $\phi_{g}=\left(\phi_{i j}(g)\right)$ then,

$$
\chi_{\phi}(g)=\sum_{i=1}^{n} \phi_{i i}(g) .
$$

Remark 2.4.2. For a degree one representation $\phi: G \rightarrow \mathbb{C}^{*}, \chi_{\phi}=\phi$.

Proposition 2.4.3. Let $\phi$ be a representation of $G$. Then, $\chi_{\phi}(1)=\operatorname{deg} \phi$.

Proof. Note that, $\chi_{\phi}(1)=\operatorname{Tr}\left(\phi_{1}\right)=\operatorname{Tr}(I)=\operatorname{dim}(V)=\operatorname{deg} \phi$.

Proposition 2.4.4. Equivalent representations have same characters. In other words, Character depends only on the equivalence class of representation.

Proof. Suppose $\phi$ and $\psi$ are two equivalent representations. Then, there exists an invertible map $\mathrm{T}$ such that, $\phi_{g}=T \psi_{g} T^{-1}$. Therefore,

$$
\chi_{\phi}(g)=\operatorname{Tr}\left(\phi_{g}\right)=\operatorname{Tr}\left(T \psi_{g} T^{-1}\right)=\operatorname{Tr}\left(T T^{-1} \psi_{g}\right)=\operatorname{Tr}\left(\psi_{g}\right)=\chi_{\psi}(g) .
$$

(Here, we used the property that $\operatorname{Tr}(\mathrm{AB})=\operatorname{Tr}(\mathrm{BA})$.

Proposition 2.4.5. Let $\phi$ be a representation of $G$. Then, $\forall g, h \in G, \chi_{\phi}(g)=$ $\chi_{\phi}\left(h g h^{-1}\right)$.

Proof.

$$
\chi_{\phi}\left(h g h^{-1}\right)=\operatorname{Tr}\left(\phi_{h g h^{-1}}\right)=\operatorname{Tr}\left(\phi_{h} \phi_{g} \phi_{h}^{-1}\right)=\operatorname{Tr}\left(\phi_{h} \phi_{h}^{-1} \phi_{g}\right)=\operatorname{Tr}\left(\phi_{g}\right)=\chi_{\phi}(g) .
$$

Definition 2.4.6. A function $f: G \rightarrow \mathbb{C}$ is called Class Function if $f(g)=$ $f\left(h g h^{-1}\right) \forall g, h \in G$ or more precisely, if $f$ is constant on conjugacy classes of $G$. The space of class functions is denoted by $Z(\mathscr{A}(G))$ In particular, characters are class functions.

Note 2.4.7. If $f: G \rightarrow \mathbb{C}$ is a class function and $C$ is the conjugacy class then, $f(C)$ will denote the constant value that $f$ takes on $C$. 
Proposition 2.4.8. $Z(\mathscr{A}(G))$ is a subspace of $\mathscr{A}(G)$.

Proof. Let $f, g \in Z(\mathscr{A}(G))$. Let $c_{1}, c_{2} \in \mathbb{C}$. Then,

$$
\begin{aligned}
\left(c_{1} f_{1}+c_{2} f_{2}\right)\left(h g h^{-1}\right) & =c_{1} f_{1}\left(h g h^{-1}\right)+c_{2} f_{2}\left(h g h^{-1}\right) \\
& =c_{1} f_{1}(g)+c_{2} f_{2}(g) \\
& =\left(c_{1} f_{1}+c_{2} f_{2}\right)(g) .
\end{aligned}
$$

Therefore, $c_{1} f_{1}+c_{2} f_{2} \in Z(\mathscr{A}(G))$ and hence, it is a subspace.

Definition 2.4.9. $C l(G)$ denotes the set of all conjugacy classes of $G$. For $C \in C l(G), \delta_{C}: G \rightarrow \mathbb{C}$ is defined as,

$$
\delta_{C}= \begin{cases}1 & ; g \in C \\ 0 & ; g \notin C .\end{cases}
$$

Proposition 2.4.10. $B=\left\{\delta_{C} \mid C \in C l(G)\right\}$ is a basis for $Z(\mathscr{A}(G))$. As a result, $\operatorname{dim}(Z(\mathscr{A}(G))=|C l(G)|$.

Proof. Note that each $\delta_{C}$ is constant on conjugacy classes. Therefore, it is a class function. First we show that B spans $Z(\mathscr{A}(G))$. Let $f \in Z(\mathscr{A}(G))$. Then, $f=\sum_{C \in C l(G)} f(C) \delta_{C}$ since, $f(g)=\sum f(C) \delta_{C}(g)=f(C)$ if $g \in C$ and then, $\mathrm{f}(\mathrm{C})=\mathrm{f}(\mathrm{g})$. Next, we show linear independence. For that, we show orthogonality. For $C, C^{\prime} \in C l(G)$,

$$
\frac{1}{O(G)} \sum_{g \in G} \delta_{C}(g) \overline{\delta_{C^{\prime}}(g)}= \begin{cases}|C| /|G| & ; C=C^{\prime} \\ 0 & ; C \neq C^{\prime}\end{cases}
$$

where $\frac{1}{O(G)} \sum_{g \in G} \delta_{C}(g) \overline{\delta_{C^{\prime}}(g)}=\left\langle\delta_{C}, \delta_{C^{\prime}}\right\rangle$. This proves the orthogonality and hence the linear independence. Therefore, $\mathrm{B}$ is a basis. Moreover, $|B|=|C l(G)|=$ number of conjugacy classes $=\operatorname{dim}(Z(\mathscr{A}(G)))$.

Theorem 2.4.11. First Orthogonality Relations: Let $\phi$ and $\psi$ be two representations of a finite group $G$. Then,

$$
\left\langle\chi_{\phi}, \chi_{\psi}\right\rangle= \begin{cases}1 & ; \phi \sim \psi \\ 0 & ; \phi \nsim \psi\end{cases}
$$

In other words, the irreducible characters of $G$ form an orthonormal set of class functions. 
Proof. Without loss of generality, assume that $\phi: G \rightarrow U_{n}(\mathbb{C})$ and $\psi: G \rightarrow$ $U_{m}(\mathbb{C})$ are unitary (since, every representation is equivalent to a unitary one.) Now, if $\phi$ and $\psi$ are equivalent, then, $\chi_{\phi}=\chi_{\psi}$. Consider, $\left\langle\chi_{\phi}, \chi_{\psi}\right\rangle$.

$$
\begin{aligned}
\left\langle\chi_{\phi}, \chi_{\psi}\right\rangle & =\frac{1}{O(G)} \sum_{g \in G} \chi_{\phi}(g) \overline{\chi_{\psi}(g)} \\
& =\frac{1}{O(G)} \sum_{g \in G} \sum_{i=1}^{n} \phi_{i i}(g) \sum_{j=1}^{m} \overline{\psi_{j j}(g)} \\
& =\sum_{i=1}^{n} \sum_{j=1}^{m} \frac{1}{O(G)} \sum_{g \in G} \phi_{i i}(g) \overline{\psi_{j j}(g)} \\
& =\sum_{i=1}^{n} \sum_{j=1}^{m}\left\langle\phi_{i i}(g), \psi_{j j}(g)\right\rangle .
\end{aligned}
$$

Now, by Schur's orthogonality Relations, $\left\langle\phi_{i i}(g), \psi_{j j}(g)\right\rangle=0$ if $\phi \not \psi$. This implies, $\left\langle\chi_{\phi}, \chi_{\psi}\right\rangle=0$ if $\phi \nsim \psi$. If $\phi \sim \psi$, assume $\phi=\psi$ then, by Schur's Orthogonality Relation,

$$
\left\langle\phi_{i i}, \psi_{j j}\right\rangle= \begin{cases}1 / n & ; i \neq j \\ 0 & ; i=j .\end{cases}
$$

Therefore,

$$
\begin{aligned}
\left\langle\chi_{\phi}, \chi_{\phi}\right\rangle & =\sum_{i=1}^{n}\left\langle\phi_{i i}, \phi_{i i}\right\rangle \\
& =\frac{1}{n} \times n=1 .
\end{aligned}
$$

Hence, irreducible characters form an orthonormal set of class functions.

Corollary 2.4.1. There are atmost $|C l(G)|$ equivalence classes of irreps of G.

Proof. By previous theorem, we know that inequivalent irreps have distinct characters and form an orthonormal set. Therefore, $\operatorname{dim}(Z(\mathscr{A}(G))=|C l(G)|$ and the orthonormal sets are linearly independent. Also, we know that, the cardinality of an orthonormal set is less than or equal to the dimension of the space. So there exist atmost $|C l(G)|$ equivalence classes of irreps of $\mathrm{G}$ (if we consider equivalence relation to be conjugation). 
Notation: $m V=V \oplus V \ldots \oplus V$ (m-times) and $m \phi=\phi \oplus \ldots \oplus \phi$ (m-times). Let $\phi_{i}$ denote the unitary representation of $\mathrm{G}$ and $d_{i}$ denotes the respective degrees.

Definition 2.4.12. If $\psi \sim m_{1} \phi_{1} \oplus m_{2} \phi_{2} \oplus \ldots \oplus m_{s} \phi_{s}$ then, $m_{i}$ is the multiplicity of $\phi_{i}$ in $\psi$. If $m_{i}>0$ then, $\phi_{i}$ is irreducible constituent of $\psi$.

Note that, since the character depends only on the equivalence class so multiplicity of $\phi_{i}$ will be same, no matter what the decomposition of $\psi$ is.

Remark 2.4.13. If $\psi \sim m_{1} \phi_{1} \oplus m_{2} \phi_{2} \oplus \ldots \oplus m_{s} \phi s$ then, deg $\psi=m_{1} d_{1}+$ $m_{2} d_{2}+\ldots+m_{s} d_{s}$.

Lemma 2.4.14. Let $\phi=\rho \oplus \psi$ then, $\chi_{\phi}=\chi_{\rho}+\chi_{\psi}$.

Proof. Proof: Let $\rho: G \rightarrow G L_{n}(\mathbb{C})$ and $\psi: G \rightarrow G L_{m}(\mathbb{C})$ then, $\phi: G \rightarrow$ $G L_{n+m}(\mathbb{C})$ has the block form as,

$$
\phi_{g}=\left(\begin{array}{cc}
\rho_{g} & 0 \\
0 & \psi_{g}
\end{array}\right)
$$

$\chi_{\phi}(g)=\operatorname{Tr}\left(\phi_{g}\right)=\operatorname{Tr}\left(\rho_{g}\right)+\operatorname{Tr}\left(\psi_{g}\right)=\chi_{\rho}(g)+\chi_{\psi}(g)$. This shows that,

$$
\chi_{\phi}=\chi_{\rho}+\chi_{\psi}
$$

Theorem 2.4.15. Let $\phi_{1}, \ldots, \phi_{s}$ be a complete set of representatives of equivalence classes of irreps of $G$ and let

$$
\rho \sim m_{1} \phi_{1} \oplus \ldots \oplus m_{s} \phi_{s}
$$

Then, $m_{i}=\left\langle\chi_{\rho}, \chi_{\phi_{i}}\right\rangle$. Consequently, decomposition of $\rho$ into irreducible constituents is unique and $\rho$ is determined upto equivalence by its characters.

Proof. We know that $\chi_{\rho}=m_{1} \chi_{\phi_{1}}+\ldots .+m_{s} \chi_{\phi_{s}} .\left\langle\chi_{\rho}, \chi_{\phi_{i}}\right\rangle=m_{1}\left\langle\chi_{\phi_{1}}, \chi_{\phi_{i}}\right\rangle+$ $\ldots+m_{s}\left\langle\chi_{\phi_{s}}, \chi_{\phi_{i}}\right\rangle=m_{i}$.

So, decomposition of $\rho$ into irreducible constituents is unique and if representations are equivalent then characters are same.

Hence we conclude that this theorem helps us to check whether a representation is irreducible or not. 
Corollary 2.4.2. A representation is irreducible iff $\left\langle\chi_{\rho}, \chi_{\rho}\right\rangle=1$.

Proof. Suppose a representation $\rho$ is not irreducible. Then,

$$
\rho \sim m_{1} \phi_{1} \oplus \ldots \oplus m_{s} \phi_{s} .
$$

Note that, $\chi_{\rho}=m_{1} \chi_{\phi_{1}}+\ldots .+m_{s} \chi_{\phi_{s}}$. This implies that,

$$
\left\langle\chi_{\rho}, \chi_{\rho}\right\rangle=\left\langle m_{1} \chi_{\phi_{1}}+\ldots .+m_{s} \chi_{\phi_{s}}, m_{1} \chi_{\phi_{1}}+\ldots .+m_{s} \chi_{\phi_{s}}\right\rangle
$$

$=m_{1}^{2}+m_{2}^{2}+\ldots+m_{s}^{2}$, Therefore, $\left\langle\chi_{\rho}, \chi_{\rho}\right\rangle=1$ iff there exists $\mathrm{j}$ such that $m_{j}=1$ and $m_{i}=0 \forall i \neq j$ but this is the case only when $\rho$ is irreducible.

Example 2.4.16. Consider $\rho: S_{3} \rightarrow G L_{2}(\mathbb{C})$ as

$$
\rho(12)=\left(\begin{array}{cc}
-1 & -1 \\
0 & 1
\end{array}\right) \text { and } \rho(123)=\left(\begin{array}{cc}
-1 & -1 \\
1 & 0
\end{array}\right)
$$

then, $\chi_{\rho}(I d)=2=\operatorname{deg} \rho, \chi_{\rho}(12)=0$, and $\chi_{\rho}(123)=-1$.

$\left\langle\chi_{\rho}, \chi_{\rho}\right\rangle=\frac{1}{6} \sum_{g \in G} \chi_{\rho}(g) \overline{\chi_{\rho}(g)}=\frac{1}{6}\left(2^{2}+3.0^{2}+2 \cdot(-1)^{2}\right)=1$.

Hence this representation is irreducible.

Example 2.4.17. Characters of $S_{3}:$ Trivial character : $\chi_{1}: S_{3} \rightarrow \mathbb{C}^{*}$ defined as, $\chi_{1}(\sigma)=1 \forall \sigma \in S_{3}$. Note that any degree one representation is equal to its character. Since $S_{3}$ has three conjugacy classes, this implies there should exist three inequivalent irreps of $S_{3}$. Therefore, by proposition done earlier $\sum d_{i}^{2} \leq O(G)$ which gives $1^{2}+d^{2}+2^{2} \leq 6$. In this expression '2' represents the degree two irreducible representation of $S_{3}$ considered in previous example. This shows that $d=1$.

So, we can define another degree one representation for $S_{3}$ as,

$$
\chi_{2}(\sigma)= \begin{cases}1 & ; \sigma \text { is even } \\ -1 & ; \sigma \text { is odd }\end{cases}
$$

Character Table: Rows of a character table correspond to the irreducible characters and the columns of a character table correspond to conjugacy classes.

Standard Representation of $S_{3}$ gives,

$$
\phi(12)=\left(\begin{array}{lll}
0 & 1 & 0 \\
1 & 0 & 0 \\
0 & 0 & 1
\end{array}\right) \text { and } \phi(123)=\left(\begin{array}{lll}
0 & 0 & 1 \\
1 & 0 & 0 \\
0 & 1 & 0
\end{array}\right)
$$


So, for this representation, we have the following character table:

\begin{tabular}{||cccc||}
\hline & Id & $\left(\begin{array}{lll}1 & 2\end{array}\right)$ & $\left(\begin{array}{lll}1 & 2 & 3\end{array}\right)$ \\
\hline \hline$\chi_{\phi}$ & 3 & 1 & 0 \\
\hline
\end{tabular}

Similarly, we can form character table for three inequivalent representations of $S_{3}$. Consider $\phi_{1}$ to be the trivial representation of $S_{3}$. Another, consider $\phi_{2}$ to be the one degree representation whose character is defined as,

$$
\chi_{2}(\sigma)= \begin{cases}1 & ; \sigma \text { is even } \\ -1 & ; \sigma \text { is odd }\end{cases}
$$

and lastly, consider $\phi_{3}$ as the two degree irreducible representation considered in the first example. Now the character table is as follows.

\begin{tabular}{||cccc||}
\hline & Id & $\left(\begin{array}{llll}1 & 2\end{array}\right)$ & $\left(\begin{array}{lll}1 & 2 & 3\end{array}\right)$ \\
\hline \hline$\chi_{1}$ & 1 & 1 & 1 \\
\hline$\chi_{2}$ & 1 & -1 & 1 \\
\hline$\chi_{3}$ & 2 & 0 & -1 \\
\hline
\end{tabular}

This shows that $\chi_{\phi}=\chi_{1}+\chi_{3}$. That is, $\phi \sim \chi_{1} \oplus \chi_{3}$.

We can also conclude the same by computing the following :

$\left\langle\chi_{\phi}, \chi_{1}\right\rangle=\frac{1}{6}(3+3.1+2.0)=1=m_{1}$

Similarly, $\left\langle\chi_{\phi}, \chi_{2}\right\rangle=\frac{1}{6}(3+3 .(-1)+2.0)=0=m_{2}$

and also, $\left\langle\chi_{\phi}, \chi_{3}\right\rangle=\frac{1}{6}(0+3.0+2.0)=1=m_{3}$.

We can note that columns of character table are pairwise orthogonal as can be seen in the table drawn above.

\subsection{The Regular Representation}

Another way of looking at the Regular Representation is the following: We know that by Cayley's theorem, every finite group $G$ is isomorphic to some subgroup of $S_{n}$ where n is the order of the group G. Hence, we can restrict the standard representation of $S_{n}$ that is, $\phi: G \rightarrow G L_{n}(\mathbb{C})$ to $\mathrm{G}$ and get the representation called the Regular Representation. Suppose we have a finite set X. Then,

$$
\mathbb{C} X=\left\{\sum_{x \in X} c_{x} x \mid c_{x} \in \mathbb{C}\right\}
$$


is the vector space generated with basis $\mathrm{X}$. Note that, $\sum_{x \in X} a_{x} x=\sum_{x \in X} b_{x} x$ iff $a_{x}=b_{x} \forall x \in X$. Addition here is defined as,

$$
\sum a_{x} x+\sum b_{x} x=\sum_{x \in X}\left(a_{x}+b_{x}\right) x .
$$

Scalar multiplication is defined as,

$$
\sum \alpha a_{x} x=\alpha \sum a_{x} x .
$$

The inner product is defined as,

$$
\left\langle\sum_{x \in X} a_{x} x, \sum_{x \in X} b_{x} x\right\rangle=\sum_{x \in X} a_{x} \overline{b_{x}} .
$$

Definition 2.5.1. Let $G$ be a finite group. The Regular Representation of $G$ is a homomorphism $L: G \rightarrow G L(\mathbb{C} G)$ defined by

$$
L_{g} \sum_{h \in G} c_{h} h=\sum_{h \in G} c_{h} g h=\sum_{x \in G} c_{g^{-1} x} x
$$

for $g \in G$. (By using change of variable, letting $g h=x$ ) $L_{g}$ is a linear operator acting on a linear combination of basis vectors given the action on basis.

Remark 2.5.2. The Regular Representation is never irreducible when $G$ is non trivial but contains all irreps of $G$ as constituents.

Proposition 2.5.3. The Regular Representation is Unitary Representation of $G$.

Proof. Since $L_{g}$ is linear so for $g_{1}, g_{2} \in G, h \in G$ is a basis element of $\mathbb{C}(G)$. Hence,

$$
\left(L_{g_{1}} L_{g_{2}}\right)(h)=L_{g_{1}}\left(g_{2} h\right)=g_{1} g_{2} h=L_{g_{1} g_{2}} h
$$

So, $\mathrm{L}$ is a homomorphism.

Now,

$$
\begin{aligned}
\left\langle L_{g} \sum c_{h} h, L_{g} \sum k_{h} h\right\rangle & =\left\langle\sum c_{g^{-1} x} x, \sum k_{g^{-1} x} x\right\rangle \\
& =\sum c_{g^{-1} x} \overline{k_{g^{-1}} x} .
\end{aligned}
$$

Let $y=g^{-1} x$, we get the expression equal to $\sum_{y \in G} c_{y} \overline{k_{y}}$ that is further equal to $\left\langle\sum c_{y} y, \sum k_{y} y\right\rangle$. This shows that $L_{g}$ is unitary and hence is invertible since $L_{g}^{-1}=L_{g}^{*}$. 
Proposition 2.5.4. The character of Regular Representation is:

$$
\chi_{L}(g)= \begin{cases}|G| & ; g=1 \\ 0 & ; g \neq 1\end{cases}
$$

Proof. Let $G=g_{1}, g_{2}, \ldots, g$ be a basis for $\mathbb{C}(G)$ then, $L_{g} g_{j}=g g_{j}$ where $\left[L_{g}\right]$ is the matrix with respect to $g$.

We see that

$$
\begin{aligned}
{\left[L_{g}\right]_{i j} } & = \begin{cases}1 & ; g_{i}=g g_{j} \\
0 & ; \text { otherwise }\end{cases} \\
& = \begin{cases}1 & ; g=g_{i} g_{j}^{-1} \\
0 & ; \text { otherwise }\end{cases}
\end{aligned}
$$

In particular,

$$
\left[L_{g}\right]_{i i}= \begin{cases}1 & ; g=1 \\ 0 & ; \text { otherwise }\end{cases}
$$

Therefore,

$$
\chi_{L}(g)=\operatorname{Tr}\left(L_{g}\right)= \begin{cases}|G| & ; g=1 \\ 0 & ; g \neq 1\end{cases}
$$

Hence proved.

Theorem 2.5.5. Let $L$ be a regular representation of $G$. Then, the following decomposition holds:

$$
L \sim d_{1} \phi_{1} \oplus d_{2} \phi_{2} \oplus \ldots \oplus d_{s} \phi_{s}
$$

Proof. We know that, $\psi \sim m_{1} \phi_{1} \oplus m_{2} \phi_{2} \oplus \ldots \oplus m_{s} \phi_{s}$, where $m_{i}=\left\langle\chi_{\psi}, \chi_{\phi_{i}}\right\rangle$.

Here, $\psi=L$. So,

$$
\begin{aligned}
\left\langle\chi_{L}, \chi_{\phi_{i}}\right\rangle & =\frac{1}{O(G)} \sum_{g \in G} \chi_{L}(g) \overline{\chi_{i}(g)} \\
& =\frac{1}{O(G)} O(G) \times \overline{\chi_{i}(1)} \\
& =1 \times \operatorname{deg} \phi_{i} \\
& =d_{i} .
\end{aligned}
$$

Therefore,

$$
L \sim d_{1} \phi_{1} \oplus \ldots \oplus d_{s} \phi_{s}
$$


Corollary 2.5.1. The formula $O(G)=d_{1}^{2}+d_{2}^{2}+\ldots+d_{s}^{2}$ holds.

Proof. Since by previous theorem,

$$
L \sim d_{1} \phi_{1} \oplus \ldots \oplus d_{s} \phi_{s}
$$

This implies that

$$
\chi_{L}=d_{1} \chi_{1}+\ldots+d_{s} \chi_{s}
$$

and hence,

$$
\chi_{L}=d_{1} \chi_{1}(1)+\ldots+d_{s} \chi_{s}(1)=d_{1}^{2}+\ldots+d_{s}^{2}
$$

and we know that $\chi_{L}(1)=O(G)$ and hence the result.

Theorem 2.5.6. The set $\left.B=\left\{\sqrt{d_{k}}\right\} \phi_{i j}(k) \mid 1 \leq k \leq s, 1 \leq i, j \leq d_{k}\right\}$ is an orthonormal basis for $\mathscr{A}(G)$.

Proof. We know that B is an orthonormal set by Schur's Orthogonality Relations.

Now, since $|B|=d_{1}^{2}+\ldots+d_{s}^{2}=|G|=\operatorname{dim}(\mathscr{A}(G))$ as $\left\{\sqrt{d} \phi_{i j} \mid 1 \leq i, j \leq d\right\}$ is the set of $d^{2}$ functions. Hence, this shows that B is an orthonormal basis for $\mathscr{A}(G)$.

Theorem 2.5.7. The set $\left\{\chi_{1}, \chi_{2}, \ldots, \chi_{s}\right\}$ is an orthonormal basis for $Z(\mathscr{A}(G))$.

Proof. We know that the irreducible characters form an orthonormal set of class functions. Now we need to show that this set forms a basis that is, it spans $Z(\mathscr{A}(G))$.

Let $f \in Z(\mathscr{A}(G))$. Now, f can be written as

$$
f=\sum_{i, j, k} c_{i j}^{(k)} \phi_{i j}(k)
$$

for some $c_{i j}^{(k)} \in \mathbb{C} ; 1 \leq k \leq s, 1 \leq i, j \leq d_{k}$. Since $\mathrm{f}$ is a class function, for 
any $x \in G$, we have

$$
\begin{aligned}
f(x) & =\frac{1}{O(G)} \sum_{g \in G} f\left(g^{-1} x g\right) \\
& =\frac{1}{O(G)} \sum_{g \in G} \sum_{i, j, k} c_{i j}^{(k)} \phi_{i j}^{(k)}\left(g^{-1} x g\right) \\
& =\sum_{i, j, k} c_{i j}^{k}\left[\frac{1}{O(G)} \sum_{g \in G} \phi_{g^{-1}}^{(k)} \phi_{x}^{(k)} \phi_{g}^{(k)}\right]_{i j} \\
& =\sum_{i, j, k} c_{i j}^{(k)}\left[\left(\phi_{x}^{(k)}\right)^{\#}\right]_{i j} \\
& =\sum_{i, j, k} c_{i j}^{(k)} \frac{\operatorname{Tr}\left(\phi_{x}^{(k)}\right)}{\operatorname{deg} \phi^{(k)}} I_{i j} \\
& =\sum_{i, k} c_{i i}^{(k)} \frac{1}{d_{k}} \chi_{k}(x) .
\end{aligned}
$$

Therefore, $f=\sum_{i, k} c_{i i}^{(k)} \frac{1}{d_{k}} \chi_{k}$. This implies, $f \in \operatorname{span}\left\{\chi_{1}, \ldots, \chi_{s}\right\}$ and $\left\{\chi_{1}, \ldots, \chi_{s}\right\}$ is an orthonormal set. Hence, $\left\{\chi_{1}, \ldots, \chi_{s}\right\}$ is an orthonormal basis for $Z(\mathscr{A}(G))$.

Corollary 2.5.2. Number of equivalence classes of irreps of $G$ is equal to the number of conjugacy classes of $G$.

Proof. Using above theorem, $s=\operatorname{dim}(Z(\mathscr{A}(G)))=|C l(G)|$.

Theorem 2.5.8. A finite group $G$ is abelian iff it has $|G|$ quivalence classes.of irreps of $G$.

Proof. A finite group $\mathrm{G}$ is abelian iff $|G|=|C l(G)|=$ number of equivalence classes of irreps of $\mathrm{G}$.

Example 2.5.9. Irreducible Representations of $\frac{\mathbb{Z}}{n \mathbb{Z}}$

$$
\begin{aligned}
\text { Let } \omega_{n}=\exp 2 \pi i / n . \quad & \text { Define } \chi_{k}: \frac{\mathbb{Z}}{n \mathbb{Z}} \rightarrow \mathbb{C}^{*} \text { by, } \\
& \chi_{k}([m])=\omega_{n}^{k m} ; 0 \leq k \leq n-1
\end{aligned}
$$


Then, $\chi_{0}, \ldots, \chi_{n-1}$ are distinct irreps of $\frac{\mathbb{Z}}{n \mathbb{Z}}$.

Following is the character table of $\frac{\mathbb{Z}}{n \mathbb{Z}}$ :

\begin{tabular}{||ccccc||}
\hline & {$[0]$} & {$[1]$} & {$[2]$} & {$[3]$} \\
\hline \hline$\chi_{1}$ & 1 & 1 & 1 & 1 \\
\hline$\chi_{2}$ & 1 & -1 & 1 & -1 \\
\hline$\chi_{3}$ & 1 & $i$ & -1 & $i$ \\
\hline$\chi_{4}$ & 1 & $-i$ & -1 & $i$ \\
\hline
\end{tabular}

Theorem 2.5.10. Second Orthogonality Relations

Let $C, C^{\prime}$ be the conjugacy classes of $G$ and let $g \in C, h \in C^{\prime}$ then,

$$
\sum_{i=1}^{s} \chi_{i}(g) \overline{\chi_{i}(h)}= \begin{cases}|G| /|C| & ; C=C^{\prime} \\ 0 & ; C \neq C^{\prime}\end{cases}
$$

Consequently, columns of character table are orthogonal and hence character table is invertible.

Proof. Consider $\delta_{C^{\prime}}$.

$$
\begin{aligned}
\delta_{C^{\prime}}(g) & =\sum_{i=1}^{s}\left\langle\delta_{C^{\prime}}, \chi_{i}\right\rangle \chi_{i}(g) \\
& =\sum_{i=1}^{s} \frac{1}{O(G)} \sum_{x \in G} \delta_{C^{\prime}}(x) \overline{\chi_{i}(x)} \chi_{i}(g) \\
& =\sum_{i=1}^{s} \frac{1}{O(G)} \sum_{x \in C^{\prime}} \overline{\chi_{i}(x)} \chi_{i}(g) \\
& =\frac{|C|}{|G|} \sum_{i=1}^{s} \chi_{i}(g) \overline{\chi_{i}(h)}
\end{aligned}
$$

(Note that LHS $=1$ if $g \in C^{\prime}$ ). So,

$$
\sum_{i=1}^{s} \chi_{i}(g) \overline{\chi_{i}(h)}= \begin{cases}|G| /|C| & ; C=C^{\prime} \\ 0 & ; C \neq C^{\prime}\end{cases}
$$


Therefore, columns of Character table form an orthogonsl set of non zero vectors and hence are linearly independent. So, Character table is invertible.

\subsection{Representation of Finite Abelian Groups}

Proposition 2.6.1. Let $G_{1}, G_{2}$ be abelian groups and suppose $\rho_{1}, \ldots ., \rho_{m}$ and $\phi_{1}, \ldots, \phi_{n}$ be irreps of $G_{1}$ and $G_{2}$ respectively. Let $m=\left|G_{1}\right|$ and $n=\left|G_{2}\right|$. Then, $\alpha_{i j}: G_{1} \times G_{2} \rightarrow \mathbb{C}^{*} ; 1 \leq i \leq m$ and $1 \leq j \leq n$ is given by:

$$
\alpha_{i j}\left(g_{1} g_{2}\right)=\rho_{i}\left(g_{1}\right) \phi_{j}\left(g_{2}\right)
$$

form complete set of irreps of $G_{1} \times G_{2}$.

Proof. First, we need to check that $\alpha_{i j}$ are homomorphisms.

$$
\begin{aligned}
\alpha_{i j}\left(g_{1}, g_{2}\right) \alpha_{i j}\left(g_{1}^{\prime}, g_{2}^{\prime}\right) & =\rho_{i}\left(g_{1}\right) \phi_{j}\left(g_{2}\right) \rho_{i}\left(g_{1}^{\prime}\right) \phi_{j}\left(g_{2}^{\prime}\right) \\
& =\rho_{i}\left(g_{1} g_{1}^{\prime}\right) \phi_{j}\left(g_{2} g_{2}^{\prime}\right) \\
& =\alpha_{i j}\left(g_{1} g_{1}^{\prime}, g_{2} g_{2}^{\prime}\right) \\
& =\alpha_{i j}\left(\left(g_{1}, g_{2}\right)\left(g_{1}^{\prime}, g_{2}^{\prime}\right)\right) .
\end{aligned}
$$

Next, we check if $\alpha_{i j}=\alpha_{k l}$ then $\mathrm{i}=\mathrm{k}$ and $\mathrm{j}=\mathrm{l}$.

Let $\alpha_{i j}=\alpha_{k l}$. Then,

$$
\rho_{i}(g)=\alpha_{i j}(g, 1)=\alpha_{k l}(g, 1)=\rho_{k}(g)
$$

This shows that $\mathrm{i}=\mathrm{k}$. Similarly, $\mathrm{j}=\mathrm{l}$.

Now, since, $G_{1} \times G_{2}$ has $\left|G_{1} \times G_{2}\right|=m n$ distinct irreps. Therefore, $\alpha_{i j}$ are all of them, where, $1 \leq i \leq m$ and $1 \leq j \leq n$.

Example 2.6.2. Character table of Klein's Four group $\mathbb{K}_{4} \cong \mathbb{Z}_{2} \times \mathbb{Z}_{2}$ Character table of $\mathbb{Z} / 2 \mathbb{Z}$ :

\begin{tabular}{||ccc||}
\hline & {$[0]$} & {$[1]$} \\
\hline \hline$\chi_{1}$ & 1 & 1 \\
\hline$\chi_{2}$ & 1 & -1 \\
\hline
\end{tabular}.


Now, character table of $\mathbb{Z} / 2 \mathbb{Z} \times \mathbb{Z} / 2 \mathbb{Z}$ using above proposition is :

\begin{tabular}{||ccccc||}
\hline & $([0],[0])$ & $([0],[1])$ & $([1],[0])$ & $([1],[1])$ \\
\hline \hline$\alpha_{11}$ & 1 & 1 & 1 & 1 \\
\hline$\alpha_{12}$ & 1 & -1 & 1 & -1 \\
\hline$\alpha_{21}$ & 1 & 1 & -1 & -1 \\
\hline$\alpha_{22}$ & 1 & -1 & -1 & 1 \\
\hline
\end{tabular}




\section{Chapter 3}

\section{Fourier Analysis on Groups}

\subsection{Fourier Analysis on Finite Groups}

Definition 3.1.1. A function $f: \mathbb{Z} \rightarrow \mathbb{C}$ is said to be periodic with period $n$ if $f(x)=f(x+n) \forall x \in \mathbb{Z}$.

From the above definition, we can conclude that these functions are in one-one correspondence with the elements of $\mathscr{A}(\mathbb{Z} / n \mathbb{Z})$ that is, the functions $f: \mathbb{Z} / n \mathbb{Z} \rightarrow \mathbb{C}$. In other words, these functions are constant o the residue classes modulo n. Now, as we know from previous chapter that the irreducible charactersform basis for $\mathscr{A}(\mathbb{Z} / n \mathbb{Z})$ that is, if $\mathrm{f}$ is a function in $\mathscr{A}(\mathbb{Z} / n \mathbb{Z})$ then,

$$
f=\left\langle f, \chi_{0}\right\rangle \chi_{0}+\ldots+\left\langle f, \chi_{n-1}\right\rangle \chi_{n-1}
$$

where $\chi_{k}([m])=e^{2 \pi i k m / n}$.

Definition 3.1.2. Fourier Transform: Let $f: \mathbb{Z} / n \mathbb{Z} \rightarrow \mathbb{C}$. Then, the fourier transform of $f$ is defined by,

$$
\hat{f}: \mathbb{Z} / n \mathbb{Z} \rightarrow \mathbb{C}
$$

as

$$
\hat{f}([m])=n\left\langle f, \chi_{m}\right\rangle=\sum_{k=0}^{n-1} f([k]) e^{-2 \pi i k m / n} .
$$

Proposition 3.1.3. The fourier transform is invertible. In simple words,

$$
f=\frac{1}{n} \sum_{k=0}^{n-1} \hat{f}([k]) \chi_{k} .
$$


Proposition 3.1.4. The class functions form the center of $\mathscr{A}(G)$.

Proof. First, Let $f: G \rightarrow \mathbb{C}$ be a class function. Then to show that,

$$
a * f=f * a \quad \forall a \in \mathscr{A}(G) \text {. }
$$

Let $a \in \mathscr{A}(G)$. Then,

$$
a * f(x)=\sum_{y \in G} a\left(x y^{-1}\right) f(y)=\sum_{y \in G} a\left(x y^{-1}\right) f\left(x y x^{-1}\right)
$$

since $\mathrm{f}$ is a class function. Now, let $x y^{-1}=z$. This implies that,

$$
a * f(x)=\sum_{z \in G} a(z) f\left(x z^{-1}\right)=\sum f\left(x z^{-1}\right) a(z)=f * a(x) .
$$

Therefore, $\mathrm{a}^{* \mathrm{f}}=\mathrm{f}^{*} \mathrm{a}$. Now we show the converse part. That is, let $f \in$ $Z(\mathscr{A}(G))$. Then, to show that $\mathrm{f}$ is a class function. So, the claim is to prove

$$
f(g h)=f(h g) \forall g, h \in G .
$$

Now

$$
\begin{aligned}
f(g h) & =\sum_{y \in G} f\left(g y^{-1}\right) \delta_{h^{-1}}(y) \\
& =f * \delta_{h^{-1}}(g) \\
& =\delta_{h^{-1}} * f(g) \\
& =\sum_{y \in G} \delta_{h^{-1}}\left(g y^{-1}\right) f(y) \\
& =f(h g)
\end{aligned}
$$

since, $\delta_{h^{-1}}\left(g y^{-1}\right)$ is non zero iff $g y^{-1}=h^{-1}$ that is, $\mathrm{y}=\mathrm{hg}$. Now, by claim we have, $f\left(g h g^{-1}\right)=f(h)$. Hence $\mathrm{f}$ is a class function.

\subsection{Fourier Analysis o finite abelian groups}

Note that if $\mathrm{G}$ is abelian then, $\mathscr{A}(G)=Z(\mathscr{A}(G))$. That is, $\mathscr{A}(G)$ is a commutative ring. 
Definition 3.2.1. Let $G$ be a finite abelian group. Then, $\hat{G}$ is the set of all irreducible characters $\chi: G \rightarrow \mathbb{C}^{*}$ known as the dual group.

Proposition 3.2.2. Let $G$ be a finite abelian group. We define product on $\hat{G}$ via pointwise multiplication that is, $(\chi \cdot \theta)=\chi(g) \theta(g)$. Then, $\hat{G}$ is an abelian group of order $|G|$ with respect to this binary operation.

Proof. Let $\chi, \theta \in \hat{G}$. Then,

$$
\begin{aligned}
\chi \cdot \theta\left(g_{1} g_{2}\right) & =\chi\left(g_{1} g_{2}\right) \theta\left(g_{1} g_{2}\right) \\
& =\chi\left(g_{1}\right) \chi\left(g_{2}\right) \theta\left(g_{1}\right) \theta\left(g_{2}\right) \\
& =\chi\left(g_{1}\right) \theta\left(g_{1}\right) \chi\left(g_{2}\right) \theta\left(g_{2}\right) \\
& =(\chi \cdot \theta)\left(g_{1}\right) \cdot(\chi \cdot \theta)\left(g_{2}\right) .
\end{aligned}
$$

This shows that $\hat{G}$ is closed under pointwise product. Trivially, this product is associative and commutative. Identity is, $\chi_{1}(g)=1 \forall g \in G$. Inverse is, $\chi^{-1}(g)=\chi(g)^{-1}=\overline{\chi(g)}$ since $\chi$ is unitary. Trivially, $\chi \cdot \chi^{-1}=\chi_{1}$. And hence, $\hat{G}$ is an abelian group. The number of irreducible characters of $\mathrm{G}$ is equal to $|G|$ where $\mathrm{G}$ is abelian. Therefore, $|\hat{G}|=|G|$.

Example 3.2.3. Let $G=\mathbb{Z} / n \mathbb{Z}$ and $\hat{G}=\left\{\chi_{0}, \ldots, \chi_{n-1}\right\}$. Let $\chi_{k}([m])=$ $e^{2 \pi i k m / n}$. Then, $[k] \rightarrow \chi_{k}$ is a group isomorphism from $G \rightarrow \hat{G}$. So, $G \cong \hat{G}$.

Definition 3.2.4. Fouier Transform: Let $f: G \rightarrow \mathbb{C}$ be a complex valued function on a finite abelian group $G$. Then, the fourier transform $\hat{f}: G \rightarrow \mathbb{C}$ is defined by

$$
\hat{f}(x)=|G|\langle f, \chi\rangle=\sum_{g \in G} f(g) \overline{\chi(g)}
$$

where, $|G|\langle f, \chi\rangle$ are the complex numbers called the fourier coefficients of $f$.

Example 3.2.5. If $\chi, \theta \in \hat{G}$ then,

$$
\hat{\chi}(\theta)=|G|\langle\chi, \theta\rangle= \begin{cases}|G| & ; \chi=\theta \\ 0 & ; \text { otherwise }\end{cases}
$$

by orthogonality relations and so, $\hat{\chi}=|G| \delta_{\chi}$.

Theorem 3.2.6. If $f \in \mathscr{A}(G)$ then,

$$
f=\frac{1}{O(G)} \sum_{x \in \hat{G}} \hat{f}(\chi) \chi
$$


Proof.

$$
f=\sum_{x \in \hat{G}}\langle f, \chi\rangle \chi=\frac{1}{O(G)} \sum_{x \in \hat{G}}|G|\langle f, \chi\rangle \chi=\frac{1}{O(G)} \sum \hat{f}(\chi) \chi
$$

Hence proved.

Proposition 3.2.7. The map $T: \mathscr{A}(G) \rightarrow \mathscr{A}(\hat{G})$ given as $T f=\hat{f}$ is invertible linear transformation.

Proof. Let $|G|=n$. $T\left(c_{1} f_{1}+c_{2} f_{2}\right)=c_{1} f_{1} \hat{+} c_{2} f_{2}$. Now, consider

$$
\begin{aligned}
c_{1} f_{1} \hat{+} c_{2} f_{2}(\chi) & =n\left\langle c_{1} f_{1}+c_{2} f_{2}, \chi\right\rangle \\
& =c_{1} n\left\langle f_{1}, \chi\right\rangle+c_{2} n\left\langle f_{2}, \chi\right\rangle \\
& =c_{1} \hat{f}_{1}(\chi)+c_{2} \hat{f}_{2}(\chi) .
\end{aligned}
$$

This shows that $c_{1} f_{1} \hat{+} c_{2} f_{2}=c_{1} \hat{f}_{1}+c_{2} \hat{f}_{2}$. Hnece, $\mathrm{T}$ is linear. By previous theorem, $\mathrm{T}$ is injective and thus invertible since $\operatorname{dim}(\mathscr{A}(G)=n=$ $\operatorname{dim}(\mathscr{A}(\hat{G}))$.

Theorem 3.2.8. The Fourier Transform satisfies $a \hat{*} b=\hat{a} * \hat{b}$. Consequently, kinear map $T: \mathscr{A}(G) \rightarrow \mathscr{A}(\hat{G})$ given by $T f=\hat{f}$ provides a ring isomorphism between $(\mathscr{A}(G),+, *)$ and $(\mathscr{A}(\hat{G}),+,$.$) .$

Proof. We know by previous theorem that $T: \mathscr{A}(G) \rightarrow \mathscr{A}(\hat{G})$ is an isomorphism of vector spaces. Therefore, it suffices to show that it is a ring homomorphism. That is, we need to show that, $T(a * b)=T a$.Tb. In other words, we need to show,

$$
a \hat{*} b=\hat{a} . \hat{b} .
$$

Let $\mathrm{n}$ be the order of $\mathrm{G}$. Consider,

$$
\begin{aligned}
a \hat{*} b(\chi) & =n\langle a * b, \chi\rangle \\
& =n \frac{1}{n} \sum_{x \in G}(a * b)(x) \overline{\chi(x)} \\
& =\sum_{x \in G} \overline{\chi(x)} \sum_{y \in G} a\left(x y^{-1}\right) b(y) \\
& =\sum_{y \in G} b(y) \sum_{x \in G} a\left(x y^{-1}\right) \overline{\chi(x)} .
\end{aligned}
$$


Let $z=x y^{-1}$. Therefore, the expression is now equal to

$$
\begin{aligned}
\sum_{y \in G} b(y) \sum_{z \in G} a(z) \overline{\chi(z y)} & =\sum_{y \in G} b(y) \overline{\chi(y)} \sum_{y \in G} b(y) \overline{\chi(y)} \\
& =n\langle a, \chi\rangle \cdot n\langle b, \chi\rangle \\
& =\hat{a} . \hat{b} .
\end{aligned}
$$

Hence proved.

Example 3.2.9. The periodic functions on $\mathbb{Z}:$ Let $f, g: \mathbb{Z} \rightarrow \mathbb{C}$ have period n. Their convolution is defined as,

$$
(f * g)(m)=\sum_{k=0}^{n-1} f(m-k) g(k) .
$$

The fourier transform is then,

$$
\hat{f}(m)=\sum_{k=0}^{n-1} f(k) e^{-2 \pi i k m / n} .
$$

Hence, following is the fourier inversion theorem.

$$
f(m)=\frac{1}{n} \sum_{k=0}^{n-1} \hat{f}(k) e^{2 \pi i k m / n} .
$$

Now, we have a look at abelian case in a different way.

Suppose $G$ is a finite abelian group with irreducible caracters $\chi_{1}, \chi_{2}, \ldots, \chi_{n}$. Then, to each function $f: G \rightarrow \mathbb{C}$, we associate its vector of fourier coefficients.

Define $T: \mathscr{A}(G) \rightarrow \mathbb{C}^{n}$ by

$$
\begin{aligned}
T f= & \left(n\left\langle f, \chi_{1}\right\rangle, n\left\langle f, \chi_{2}\right\rangle, \ldots, n\left\langle f, \chi_{n}\right\rangle\right) \\
= & \left(\hat{f}\left(\chi_{1}\right), \hat{f}\left(\chi_{2}\right), \ldots, \hat{f}\left(\chi_{n}\right)\right) .
\end{aligned}
$$

This shows that $\mathrm{T}$ is injective by fourier inversion theorem because we can ecover $\hat{f}$ and hence $\mathrm{f}$ from Tf. Also, $\mathrm{T}$ is linear and this implies that, $\mathrm{T}$ is a 
vector space isomorphism since $\operatorname{dim}(\mathscr{A}(G))=n$.

In fact, $\mathrm{T}$ is a ring isomorphism since

$$
\begin{aligned}
T(a * b) & =\left(a \hat{*} b\left(\chi_{1}\right), \ldots, a \hat{*} b\left(\chi_{n}\right)\right)=\left(\hat{a}\left(\chi_{1}\right) \hat{b}\left(\chi_{1}\right), \ldots, \hat{a}\left(\chi_{n}\right) \hat{b}\left(\chi_{n}\right)\right) \\
& =\left(\hat{a}\left(\chi_{1}\right), \ldots, \hat{a}\left(\chi_{n}\right)\right)\left(\hat{b}\left(\chi_{1}\right), \ldots, \hat{b}\left(\chi_{n}\right)\right)=\text { Ta.Tb. }
\end{aligned}
$$

Earlier we saw that $a \hat{*} b=\hat{a} . \hat{b}$. Hence, we can conclude that, If $\mathrm{G}$ is a finite abelian group of order $n$. Then,

$$
\mathscr{A}(G) \cong \mathbb{C}^{n}
$$

where $\mathbb{C}^{n}$ has structure of direct product of rings where multiplication is coordinate wise. So, for non abelian groups we must replace $\mathbb{C}$ by matrix rings over $\mathbb{C}$.

Definition 3.2.10. Define the map

$$
T: \mathscr{A}(G) \rightarrow M_{d_{1}}(\mathbb{C}) \times \ldots \times M_{d_{s}}(\mathbb{C})
$$

by

$$
T f=\left(\hat{f}\left(\phi^{1}\right), \ldots, \hat{f}\left(\phi^{s}\right)\right)
$$

where

$$
\hat{f}\left(\phi_{k}\right)_{i j}=n\left\langle f, \phi_{i j}^{(k)}\right\rangle=\sum_{g \in G} f(g) \overline{\phi_{i j}^{k}(g)} .
$$

Here Tf is called the fourier transform of $f$. Therefore, $\hat{f}\left(\phi^{k}\right)=\sum_{g \in G} f(g) \overline{\phi_{g}^{k}}$. Theorem 3.2.11. Fourier Inversion: Let $f: G \rightarrow \mathbb{C}$ be a complex valued function on $G$. Then,

$$
f=\frac{1}{n} \sum_{i, j, k} d_{k} \hat{f}\left(\phi^{k}\right)_{i j} \phi_{i j}^{k}
$$

where $n$ is the order of $G$.

Proof. Since $\left\{\sqrt{d_{k}} \phi_{i j}^{k}\right\}$ is an orthonormal basis for $\mathscr{A}(G)$ and $f \in \mathscr{A}(G)$. Therefore,

$$
\begin{aligned}
f & =\sum_{i, j, k}\left\langle f, \sqrt{d_{k}} \phi_{i j}^{k}\right\rangle \sqrt{d_{k}} \phi_{i j}^{k} \\
& =\frac{1}{n} \sum_{i, j, k} d_{k} n\left\langle f, \phi_{i j}^{k}\right\rangle \phi_{i j}^{k} \\
& =\frac{1}{n} \sum_{i, j, k} d_{k} \hat{f}\left(\phi^{k}\right)_{i j} \phi_{i j}^{k}
\end{aligned}
$$


Proposition 3.2.12. The map $T: \mathscr{A}(G) \rightarrow M_{d_{1}}(\mathbb{C}) \times \ldots \times M_{d_{s}}(\mathbb{C})$ is a vector space isomorphism.

Proof. First we show that $\mathrm{T}$ is linear. That means we need to show that $T\left(c_{1} f_{1}+c_{2} f_{2}\right)=c_{1} f_{1} \hat{+} c_{2} f_{2}$, that is, to show $c_{1} f_{1} \hat{+} c_{2} f_{2}\left(\phi^{k}\right)=c_{1} \hat{f}_{1}\left(\phi^{k}\right)+$ $c_{2} \hat{f}_{2}\left(\phi^{k}\right)$. Now, for $1 \leq k \leq s$,

$$
\begin{aligned}
c_{1} f_{1} \hat{+} c_{2} f_{2}\left(\phi^{k}\right) & =\sum_{g \in G}\left(c_{1} f_{1}+c_{2} f_{2}\right)(g) \overline{\left.\phi_{g}^{(} k\right)} \\
& =c_{1} \sum_{g \in G} f_{1}(g) \overline{\phi_{g}^{k}}+c_{2} \sum_{g \in G} f_{2}(g) \overline{\phi_{g}^{k}} \\
& =c_{1} \hat{f}_{1}\left(\phi^{k}\right)+c_{2} \hat{f}_{2}\left(\phi^{k}\right) .
\end{aligned}
$$

By fourier inversion theorem, $\mathrm{T}$ is injective since

$$
\operatorname{dim}(\mathscr{A}(G))=|G|=d_{1}^{2}+\ldots+d_{s}^{2}=\operatorname{dim}\left(M_{d_{1}}(\mathbb{C}) \times \ldots \times M_{d_{s}}(\mathbb{C})\right) .
$$

Therefore, $\mathrm{T}$ is isomorphism.

Theorem 3.2.13. Wedderburn theorem The Fourier transform

$$
T: \mathscr{A}(G) \rightarrow M_{d_{1}}(\mathbb{C}) \times \ldots \times M_{d_{s}}(\mathbb{C})
$$

is an isomorphism of rings.

Proof. We know that $\mathrm{T}$ is an isomorphism of vector spaces. So, to show ring isomorphism we just need to show:

$$
T(a * b)=T a . T b
$$

That is, we need to show,

$$
a \hat{*} b\left(\phi^{k}\right)=\hat{a}\left(\phi^{k}\right) \cdot \hat{b}\left(\phi^{k}\right) \text { for } 1 \leq k \leq s
$$

Consider,

$$
\begin{aligned}
a \hat{*} b\left(\phi^{k}\right) & =\sum_{x \in G}(a * b)(x) \overline{\phi_{x}^{k}} \\
& =\sum_{x \in G} \overline{\phi_{x}^{k}} \sum_{y \in G} a\left(x y^{-1}\right) b(y) \\
& =\sum_{y \in G} b(y) \sum_{x \in G} a\left(x y^{-1}\right) \overline{\phi_{x}^{k}}
\end{aligned}
$$


Let $z=x y^{-1} \Rightarrow x=z y$. Therefore,

$$
\begin{aligned}
a \hat{*} b\left(\phi^{k}\right) & =\sum_{y \in G} b(y) \sum_{z \in G} a(z) \overline{\phi_{z y}^{k}} \\
& =\sum_{y \in G} b(y) \sum_{z \in G} a(z) \overline{\phi_{z}^{k}} \cdot \overline{\phi_{y}^{k}} \\
& =\sum_{z \in G} a(z) \overline{\phi_{z}^{k}} \sum_{y \in G} b(y) \overline{\phi_{y}^{k}} \\
& =\hat{a}\left(\phi^{k}\right) \cdot \hat{b}\left(\phi^{k}\right) .
\end{aligned}
$$

Hence it is a ring isomorphism.

Example 3.2.14. Representation theory of $S_{n}$ can be used to analyse voting. Here is an example of Diaconis. Suppose in an election, each voter needs to rank $n$ candidates on a ballot. Let the candidates be $\{1,2, \ldots, n\}$. Then to each ballot, we correspond a permutation $\sigma \in S_{n}$. For example for $n=3$, let the ballot ranks the candidates in 312 order then the corresponding permutation is

$$
\sigma=\left(\begin{array}{lll}
1 & 2 & 3 \\
3 & 1 & 2
\end{array}\right)
$$

then election corresponds to the function $f: S_{n} \rightarrow \mathbb{N}$ where $f(\sigma)$ is the number of people whose ballot corresponds to the permutation $\sigma$. 


\section{Chapter 4}

\section{Burnside's theorem}

\subsection{Number Theory}

Definition 4.1.1. Algebraic number: A complex number ' $k$ ' is called algebraic number if it is a root of a polynomial with integer coefficients. The numbers that are not algebraic are called transcendental.

Example 4.1.2. Consider the polynomial 2z-1. We see that, $1 / 2$ is a root of this polynomial. So, 1/2 is algebraic number. Similarly, consider $z^{2}$ to be another polynomial. Note that $\sqrt{2}$ is a root of this polynomial and hence is algebraic. On the other hand, $\pi$ and $e$ are transcendental numbers since they are not the roots of any polynomial with integer coefficients.

Definition 4.1.3. Algebraic integer: A complex number 'c' is called an algebraic integer if it is a root of a monic polynomial with integer coefficients. In other words, $c$ is an algebraic integer if there exists a polynomial

$$
p(z)=z^{n}+a_{n-1} z^{n-1}+\ldots+a_{0}
$$

where $a_{0}, a_{2}, \ldots, a_{n-1} \in \mathbb{Z}$ and $p(c)=0$.

Example 4.1.4. Following the above definition, we can say that any nth root of unity is an algebraic integer. Consider the polynomial $p(z)=z^{n}-\alpha$ then nth root of $\alpha$ is an algebraic integer. Also, the characteristic polynomial of any square matrix $A$ is a monic polynomial. Hence, each eigen value of the matrix $A$ is an algebraic integer.

Proposition 4.1.5. A rational number is an algebraic integer iff it is an integer. 
Proof. Let $\mathrm{r}$ be a rational number. Then, $\mathrm{r}=\mathrm{p} / \mathrm{q}$ where $p, q \in \mathbb{Z}$, qi 0 , $\operatorname{gcd}(\mathrm{p}, \mathrm{q})=1$. Let $\mathrm{r}$ is an algebraic integer. Then, it is root of a polynomial with integer coefficients say

$$
z^{k}+_{k-1} z^{l-1}+\ldots+a_{0} .
$$

Then,

$$
0=(p / q)^{k}+a_{k-1}(p / q)^{k-1}+\ldots+a_{0} .
$$

This implies,

$$
0=p^{k}+n a_{k-1} p^{k-1}+\ldots .+a_{0} q^{k}
$$

or,

$$
m^{k}=-n\left(a_{k-1} m^{k-1}+\ldots .+a_{0} q^{k-1}\right) .
$$

So, $q \mid p^{k}$. Now, as $\operatorname{gcd}(\mathrm{p}, \mathrm{q})=1$. This shows that $\mathrm{n}$ has to be 1 or -1 .Therefore, $r=p \in \mathbb{Z}$.

Lemma 4.1.6. An element $y \in \mathbb{C}$ is an algebraic integer iff there exists $y_{1}, y_{2}, \ldots, y_{t} \in \mathbb{C}$ not all zero such that

$$
y y_{i}=\sum_{j=1}^{t} a_{i j} y_{j}
$$

where $a_{i j} \in \mathbb{Z} \forall 1 \leq i \leq t$.

Proof. To prove the forward part, first let y be an algebraic integer. Then, there exists a polynomial $p(z)=z^{n}+a_{n-1} z^{n-1}+\ldots+a_{0}$ such that $\mathrm{y}$ is root of $\mathrm{p}(\mathrm{z})$. Let $y_{i}=y^{i-1}$ for $1 \leq i \leq n$. Then, for $1 \leq i \leq n-2$, we have,

$$
y y_{i}=y y^{i-1}=y^{i}=y_{i+1}
$$

and

$$
y y_{n}=y_{n+1}=y^{n}=-a_{0}-\ldots-a_{n-1} y^{n-1} .
$$

Hence, the forwars part is satisfied. Next, we prove the converse. Let $A=$ $\left(a_{i j}\right)$ and $Y=\left[y_{1}, y_{2}, \ldots, y_{t}\right]^{\prime} \in \mathbb{C}^{t}$ then, $[A Y]_{i}=\sum_{j=1}^{t} a_{i j} y_{j}=y y_{i}=y[Y]_{i}$. This implies that $\mathrm{AY}=\mathrm{yY}$ since $Y \neq 0$ (by assumption). Hence, $\mathrm{y}$ is an eigen value of $t \times t$ integer matrix A. Hence, $\mathrm{y}$ is an algebraic integer.

Corollary 4.1.1. The set $\mathbb{A}$ of algebraic integers form a subring of $\mathbb{C}$. In particular, sum and product of algebraic integers is algebraic. 
Proof. Note that if $a \in \mathbb{A}$ then, $-a \in \mathbb{A}$. Let $x, x^{\prime} \in \mathbb{A}$. We choose $x_{1}, x_{2}, \ldots x_{t} \in \mathbb{C}^{t}$ (not all zero) and $x_{1}^{\prime}, \ldots, x_{t}^{\prime} \in \mathbb{C}^{t}$ (not all zero) such that

$$
x x_{i}=\sum_{j=1}^{t} a_{i j} x_{j}, x^{\prime} x_{k}^{\prime}=\sum_{j=1}^{s} b_{k j} y_{j}^{\prime}
$$

then,

$$
\left(x+x^{\prime}\right) x_{i} x_{k}^{\prime}=x x_{i} x_{k}^{\prime}+x^{\prime} x_{k}^{\prime} x_{i}=\sum_{j=1}^{t} a_{i j} x_{j} x_{k}^{\prime}+\sum_{j=1}^{s} b_{k j} x_{j}^{\prime} x_{i}
$$

which is an integral linear combination of $x_{j} x_{l}^{\prime}$. This shows that $x+x^{\prime} \in \mathbb{A}$ Similarly, $x x^{\prime} x_{i} x_{k}^{\prime}=x x_{i} x^{\prime} x_{k}^{\prime}$ is integral combination of $x_{j} x_{l}^{\prime}$. So, $x x^{\prime} \in \mathbb{A}$. Therefore, $\mathbb{A}$ is a sunbring of $\mathbb{C}$.

Note 4.1.7. If $k$ is an algebraic integer then $\bar{k}$ is also an algebraic integer.

\subsection{The Dimension Theorem}

Corollary 4.2.1. Let $\chi$ be a character of finite group $G$. Then, $\chi(g)$ is an algebraic integer $\forall g \in G$.

Proof. Proof: Let $\phi: G \rightarrow G L_{m}(\mathbb{C})$ be a representation with character $\chi$. Let $\mathrm{n}$ be order of G. Then, $g^{n}=1$. This implies $\phi_{g}^{n}=\mathbb{I}$. Now $\phi_{g}$ is digonalizable since every representation is equivalent to a unitary representation and $\phi_{g}$ has eigen values $\lambda_{1}, \lambda_{2}, \ldots, \lambda_{m}$ which are nth roots of unity. So, eigen values of $\phi_{g}$ are algebraic integers. Now, $\chi_{g}=\operatorname{Tr}\left(\phi_{g}\right)=\lambda_{1}+\ldots+\lambda_{m}$ and set of all algebraic integers form a ring. Therefore, $\chi_{g}$ is an algebraic integer.Hence, we can conclude that $\chi_{\phi}(g)$ is a sum of $m$ nth roots of unity.

Theorem 4.2.1. Let $\phi$ be an irrep of finite group $G$ of degree d. Let $g \in G$ and let $s$ be the size of conjugacy class of $g$. Then, $s \chi_{\phi}(g) / d$ is an algebraic integer.

Proof. Let $C_{1}, C_{2}, \ldots, C_{n}$ be the conjugacy classes of G. Let $s i=\left|C_{i}\right|$ and let $\chi_{i}$ denote $\chi_{\phi}$ on $C_{i}$. We need to show that $s_{i} \chi_{i} / d$ is an algebraic integer for all i. For that, we consider the operator

$$
T_{i}=\sum_{x \in C_{i}} \phi_{x} .
$$


Our first claim is, $T_{i}=\frac{s_{i}}{d} \chi_{i} . I$.

The proof of this claim goes as follows. We make use of schur's lemma to prove this claim. First, consider $\phi_{g} T_{i} \phi_{g^{-1}}$.

$$
\begin{aligned}
\phi_{g} T_{i} \phi_{g^{-1}} & =\sum_{x \in C_{i}} \phi_{g} \phi_{x} \phi_{g^{-1}} \\
& =\sum_{x \in C_{i}} \phi_{g x g^{-1}} \\
& =\sum_{y \in C_{i}} \phi_{y} \\
& =T_{i}
\end{aligned}
$$

since $C_{i}$ is conjugacy class of $\mathrm{G}$. Now, by Schur's lemma, $T_{i}=\lambda I$ for some $\lambda \in \mathbb{C}$. Now,

$$
\operatorname{Tr}(\lambda I)=\operatorname{Tr}\left(T_{i}\right)=\sum_{x \in C_{i}} \operatorname{Tr}\left(\phi_{x}\right)=\sum_{x \in C_{i}} \chi_{\phi}(x)=\left|C_{i}\right| \chi_{i}=s_{i} \chi_{i} .
$$

Also, we know that, $\operatorname{Tr}(\lambda I)=d \lambda$. So, we get $\lambda=s_{i} \chi_{i} / d$. Hence, the claim. Our next claim is to show

$$
T_{i} T_{j}=\sum_{k=1}^{n} a_{i j k} T_{k} \text { for some } a_{i j k} \in \mathbb{Z}
$$

Proof goes as follows:

$$
T_{i} T_{j}=\sum_{x \in C_{i}} \phi_{x} \sum_{y \in C_{j}} \phi_{y}=\sum_{x \in C_{i}, y \in C_{j}} \phi_{x y}=\sum_{g \in G} a_{i j g} \phi_{g}
$$

where $a_{i j g} \in \mathbb{Z}$ is the number of ways to write $\mathrm{g}=$ xy with $x \in C_{i}$ and $y \in C_{j}$. Now, the subclaim is to show that $a_{i j g}$ depends only on conjugacy class of $\mathrm{g}$. So, to prove subclaim, we start by considering $X_{g}=\left\{(x, y) \in C_{i} \times C_{j} \mid x y=\right.$ $g\}$. From this, we can conclude that $a_{i j g}=\left|X_{g}\right|$. Let g' be conjugate to $\mathrm{g}$ then we show that number of ways to write $\mathrm{g}=\mathrm{xy}$ is same as number of ways of writing g' $=$ xy with $x \in C_{i}$ and $y \in C_{j}$ that is, we show, $\left|X_{g}\right|=\left|X_{g^{\prime}}\right|$. Let $g^{\prime}=k g k^{-1}$ since, g' is conjugate to g. Now define the bijection $\psi: X_{g} \rightarrow$ $X_{g^{\prime}}$ by $\psi(x, y)=\left(k x k^{-1}, k y k^{-1}\right)$ where $k x k^{-1} \in C_{i}$ and $k y k^{-1} \in C_{j}$ and 
$k x k^{-1} k y k^{-1}=k x y k^{-1}=k g k^{-1}=g^{\prime}$ that is, $\left(k x k^{-1}, k y k^{-1}\right) \in X_{g^{\prime}}$. This implies that, $\psi(x, y) \in X_{g^{\prime}}$. Hence, $\psi$ has inverse $\rho: X_{g^{\prime}} \rightarrow X_{g}$ given by

$$
\rho\left(x^{\prime}, y^{\prime}\right)=\left(k^{-1} x^{\prime} k, k^{-1} y^{\prime} k\right)
$$

and hence $\psi$ is a bijection and so, $\left|X_{g}\right|=\left|X_{g^{\prime}}\right|$. Therefore, the subclaim is proved. Now getting back to proof of second claim, let $a_{i j k}$ be value of $a_{i j g}$ with $g \in C_{k}$ then,

$$
\begin{aligned}
\sum_{g \in G} a_{i j g} \phi_{g} & =\sum_{k=1}^{n} \sum_{g \in C_{k}} a_{i j g} \phi_{g} \\
& =\sum_{k=1}^{n} a_{i j k} \sum_{g \in C_{k}} \phi_{g} \\
& =\sum_{k=1}^{n} a_{i j k} T_{k} .
\end{aligned}
$$

So, the second claim is done. Now, as $T_{i} T_{j}=\sum a_{i j k} T_{k}$. This implies,

$$
\left(\frac{s_{i}}{d} \chi_{i}\right)\left(\frac{s_{i}}{d} \chi_{j}\right)=\sum_{k=1}^{n} a_{i j k}\left(\frac{s_{k}}{d} \chi_{k}\right) .
$$

So, $s_{i} \chi_{i} / d$ is an algebraic integer by the equivalent condition for a complex number to be an algebraic integer stated in the lemma done before.

Theorem 4.2.2. The Dimension Theorem: Let $\phi$ be an irrep of $G$ of degree $d$. Then, $d \mid O(G)$.

Proof. By first Orthogonality Relations,

$$
1=\left\langle\chi_{\phi}, \chi_{\phi}\right\rangle=\frac{1}{O(G)} \sum_{x \in G} \chi_{\phi}(x) \overline{\chi \phi(x)} .
$$

This implies that,

$$
\frac{O(G)}{d}=\sum_{x \in G} \frac{\chi_{\phi}(x) \overline{\chi_{\phi}(x)}}{d} .
$$

Now let $C_{1}, C_{2}, \ldots, C_{n}$ be conjugacy classes of $\mathrm{G}$ and let $\chi_{i}$ denote value of $\chi_{\phi}$ on $C_{i}$. Let $s_{i}=\left|C_{i}\right|$. 


$$
\begin{aligned}
\frac{|G|}{d} & =\sum_{i=1}^{n} \sum_{g \in C_{i}} \frac{\chi_{\phi}(g)}{d} \overline{\chi_{\phi}(g)} \\
& =\sum_{i=1}^{n} \sum_{g \in C_{i}}\left(\frac{1}{d} \chi_{i}\right) \overline{\chi_{i}} \\
& =\sum_{i=1}^{n}\left(\frac{s_{i}}{d} \chi_{i}\right) \overline{\chi_{i}} .
\end{aligned}
$$

But by previous theorem, we know that $s_{i} \chi_{i} / d$ is an algebraic integer. And also, $\overline{\chi_{i}}$ is an algebraic integer. Now since, algebraic integer forms ring, this shows that $|G| / d$ is algebraic integer. Thus, it is an integer. So, $d \mid O(G)$. Hence proved.

Corollary 4.2.2. Let $p$ be prime and let $G$ be a group of order $p^{2}$. Then, $G$ is abelian.

Proof. Let $d_{1}, d_{2}, \ldots d_{n}$ be degrees of irreps of $\mathrm{G}$. Then, $d_{i}$ can be 1 , p or $p^{2}$. Since trivial representation has degree 1 and

$$
p^{2}=|G|=d_{1}^{2}+\ldots+d_{n}^{2} .
$$

This implies that $d_{i}=1 \forall i$. Hence, $\mathrm{G}$ is abelian.

Definition 4.2.3. Let $G$ be a group. Let $G$ ' be the commutator subgroup of $G$. Then, $G$ ' is generated by all elements of the form $g^{-1} h^{-1}$ gh where $g, h \in G$. The commutator subgroup of $G$ is a normal suvgroup and $G / G$ ' is abelian and if $N$ ia some other normal subgroup of $G$ such that $G / N$ is abelian then, $G^{\prime} \subseteq N$.

Lemma 4.2.4. Let $G$ be a finite group. Then, the number of degree one representations of $G$ divides $|G|$. More precisely, if $G$ 'is the commutator subgroup of $G$, there is a bijection between the degree one representations of $G$ and the irreps of abelian group $G / G^{\prime}$. Hence, $G$ has $\left|G / G^{\prime}\right|=\left[G: G^{\prime}\right]$ degree one representations.

Proof. Proof: Consider the canonical projection $\pi: G \rightarrow G / G^{\prime}$ defined by $\pi(g)=g G^{\prime}$. If $\psi: G / G^{\prime} \rightarrow \mathbb{C}^{*}$ is an irreducible representation then, $\psi \pi:$ $G \rightarrow \mathbb{C}^{*}$ is a degree one representation. We need to show that every degree 
one representation is of this form. Let $\rho: G \rightarrow \mathbb{C}^{*}$ be degree one representation. Then, $\operatorname{Im} \rho \cong G / \operatorname{Ker} \rho$ is abelian. Therefore, $G^{\prime} \subseteq \operatorname{Ker} \rho$. We define $\psi$ : $G / G^{\prime} \rightarrow \mathbb{C}^{*}$ by $\psi\left(g G^{\prime}\right)=\rho(g)$. If we take $\mathrm{gG}^{\prime}=\mathrm{hG}^{\prime}$ then, $h^{-1} g \in G^{\prime} \subseteq K \operatorname{Ker} \rho$ and so, $\rho\left(h^{-1} g\right)=1$. Thus, $\rho(h)=\rho(g)$. This implies $\psi\left(g G^{\prime}\right)=\psi\left(h G^{\prime}\right)$. So, this map is well defined. Next, we show map to be homomorphism as follows. $\psi\left(g G^{\prime} h G^{\prime}\right)=\psi\left(g h G^{\prime}\right)=\rho(g h)=\rho(g) \rho(h)=\psi\left(g G^{\prime}\right) \psi\left(h G^{\prime}\right)$. Hence, map is homomorphism. Now, note that $\rho(g)=\psi \pi(g)=\psi\left(g G^{\prime}\right) \forall g \in G$.. This shows $\rho=\psi \pi$. So, there exists bijection between degree one representations of $\mathrm{G}$ and irreps of abelian group $\mathrm{G} / \mathrm{G}^{\prime}$. Now, irreducible representations of abelian group $\mathrm{G} / \mathrm{G}^{\prime}=\left|G / G^{\prime}\right|=|G| /\left|G^{\prime}\right|=$ degree one representations. Hence, proved.

Corollary 4.2.3. Let $p$ and $q$ be primes with $p<q$ and $p \nmid q-1$ (or $q \not \equiv 1$ modp). Then, any group $G$ of order $p q$ is abelian.

Proof. Let $d_{1}, d_{2}, \ldots, d_{s}$ be degrees of irreps of G. Since, $d_{i} \mid O(G)$ and $p<q$ and $p q=|G|=d_{1}^{2}+\ldots .+d s^{2}$. This implies, $d_{i}=1$ or $p \forall i$. Let $\mathrm{n}$ be the number of degree $p$ representations of $G$ and let $m$ be the number of degree one representations of $\mathrm{G}$. Then, $p q=m+n p^{2}$. Now, since by previous lemma, number of degree one representations of $\mathrm{G}$ divides oreder of $\mathrm{G}$. So, $m \mid O(G)$. Since, there always exists a trivial representation therefore, $m \geq 1$. Now note that, $p \mid p q$ that is, $p \mid m+n p^{2} \Rightarrow m+n p^{2}=k p$ or $m=k p-n p^{2}$ that is, $m=p(k-n p)$. Hence, $p \mid m$. This shows that $\mathrm{m}=\mathrm{p}$ or $\mathrm{m}=\mathrm{pq}$. Note that $m \neq q$ since, $p \nmid q$. If $\mathrm{m}=\mathrm{p}, p q=p+n p^{2}=p\left(1+n p^{2}\right)$ that is, $q=1+n p$. This means, $p \mid q-1$ which is a contradiction. Therefore, $\mathrm{m}=\mathrm{pq}$. Hence, all irreducible representations of $\mathrm{G}$ have degree one. So, $\mathrm{G}$ is abelian.

\subsection{The Burnside's Theorem}

Recall that if we have a group of order $\mathrm{n}$ and a representation $\phi: G \rightarrow$ $G L_{d}(\mathbb{C})$. Then, $\chi_{\phi}(g)$ is sum of $\mathrm{d}$ nth roots of unity.

Lemma 4.3.1. Let $\lambda_{1}, \lambda_{2}, \ldots, \lambda_{d}$ be nth roots of unity. Then,

$$
\left|\lambda_{1}+\ldots+\lambda_{d}\right| \leq d
$$

and equality holds iff all $\lambda_{i}$ 's are equal. 
Proof. Let v,w be two vectors in $\mathbb{R}^{2}$. Then,

$$
\|v+w\|^{2}=\|v\|^{2}+2\langle v, w\rangle+\|w\|^{2}=\|v\|^{2}+2\|v\|^{2}\|w\|^{2} \cos \theta+\|w\|^{2}
$$

where $\theta$ is the angle between $\mathrm{v}$ and $\mathrm{w}$. Now $\cos \theta=1$ iff $\theta=0$. That is, $\|v+w\| \leq\|v\|+\|w\|$ and $\|v+w\|=\|v\|+\|w\|$ iff $v=\lambda w$ or $w=\lambda v$ for some $\lambda \geq 0$ ( since, $\cos \theta=1$ iff thet $a=0$ that is, $\mathrm{v}$ and $\mathrm{w}$ are scalar multiples of each other). Therefore, by induction,

$$
\left\|v_{1}+v_{2}+\ldots+v_{n}\right\| \leq\left\|v_{1}\right\|+\ldots+\left\|v_{n}\right\|
$$

and holds equality iff $v_{i}$ are non negative scalar multiples of a common vector. If we identify cmplex numbers with vectors in $\mathbb{R}^{2}$ then, we have,

$$
\left|\lambda_{1}+\ldots .+\lambda_{d}\right| \leq\left|\lambda_{1}\right|+\ldots+\left|\lambda_{d}\right|
$$

with equality iff $\lambda_{i}$ are non negative scalar multiples of some complex number z. But $\left|\lambda_{1}\right|=\ldots .=\left|\lambda_{d}\right|=1$. This shows that $\lambda_{1}=\lambda_{2}=\ldots=\lambda_{d}$ and $\left|\lambda_{1}+\ldots .+\lambda_{d}\right| \leq d$.

Let $\omega_{n}=e^{2 \pi i / n}$. Then, $Q\left[\omega_{n}\right]$ denotes smallest subfield $\mathbb{F}$ of $\mathbb{C}$ containing $\omega_{n}$.

Lemma 4.3.2. The field $Q\left[\omega_{n}\right]$ has dimension $\phi(n)$ as a $Q$-vector space.

Notation: Let $\Gamma=\operatorname{Gal}\left(Q\left[\omega_{n}\right]: Q\right)$ be the Galois group of $Q\left[\omega_{n}\right]$ over Q. That is, $\Gamma$ is the group of all field automorphisms $\sigma: Q\left[\omega_{n}\right] \rightarrow Q\left[\omega_{n}\right]$ where $Q\left[\omega_{n}\right]=\left\{f\left(\omega_{n}\right) \mid f(x) \in Q[x]\right\}$ such that $\sigma(r)=r \forall r \in Q$. Note that $|\Gamma|=\phi(n)$ since, $\operatorname{dim} Q\left[\omega_{n}\right]=\phi(n)$ as a Q-vector space.

Fact: If $\mathrm{p}(\mathrm{z})$ is a polynomial with rational coefficients then, $\Gamma$ permutes the roots of $\mathrm{p}$ in $Q\left[\omega_{n}\right]$.

Lemma 4.3.3. Let $p(z)$ be a polynomial with rational coefficients and suppose that $\alpha \in Q\left[\omega_{n}\right]$ is a root of $p$. Then, $\sigma(\alpha)$ is also root of $p \forall \sigma \in \Gamma$.

Proof. Proof: Consider the polynomial $p(z)=a_{k} z^{k}+a_{k-1} z^{k-1}+\ldots+a_{0}$ with $a_{i} \in Q$. Then,

$$
\begin{aligned}
p(\sigma(\alpha)) & =a_{k} \sigma(\alpha)^{k}+a_{k-1} \sigma(\alpha)^{k-1}+\ldots+a_{0} \\
& =\sigma\left(a_{k} \alpha^{k}+a_{k-1} \alpha^{k-1}+\ldots+a_{0}\right) \\
& =\sigma(0) \\
& =0 \text { since, } \sigma\left(a_{i}\right) \\
& =a_{i} \forall i .
\end{aligned}
$$


Corollary 4.3.1. Let $\alpha$ be an nth root of unity. Then, $\sigma(\alpha)$ is also an nth root of unity $\forall \sigma \in \Gamma$.

Remark 4.3.4. $\Gamma$ is isomorphic to $\mathbb{Z} / n \mathbb{Z}^{*}$.

Corollary 4.3.2. Let $\alpha \in Q\left[\omega_{n}\right]$ be an algebraic integer and suppose that $\sigma \in \Gamma$. Then, $\sigma(\alpha)$ is an algebraic integer.

Proof. Let $\alpha$ be the root of monic polynomial $\mathrm{p}$ with integer coefficients. Then, so is $\sigma(\alpha)$ by previous lemma.

Theorem 4.3.5. Let $\alpha \in Q\left[\omega_{n}\right]$. Then, $\sigma(\alpha)=\alpha \forall \alpha \in \Gamma$ if and only if $\alpha \in Q$.

Corollary 4.3.3. Let $\alpha \in Q\left[\omega_{n}\right]$. Then, $\prod_{\sigma \in \Gamma} \sigma(\alpha) \in Q$.

Proof: Let $\tau \in \Gamma$, Then,

$$
\tau\left(\prod_{\sigma \in \Gamma} \sigma(\alpha)\right)=\prod_{\sigma \in \Gamma}(\tau \sigma(\alpha))=\prod_{\rho \in \Gamma} \rho(\alpha)
$$

where $\rho=\tau \sigma$. Now, by previous theorem, $\prod_{\sigma \in \Gamma} \sigma(\alpha) \in Q$.

Theorem 4.3.6. Let $G$ be a group of order $n$. Let $C$ be conjugacy class of $g \in G$. Let $h$ be the order of C.Let $\phi: G \rightarrow G L_{d}(\mathbb{C})$ be irreducible representation of $G$ and $\operatorname{gcd}(h, d)=1$. Then, either, (i) there exists $\lambda \in \mathbb{C}^{*}$ such that $\phi_{g}=\lambda I \forall g \in C$ or, (ii) $\chi_{\phi}(g)=0 \forall g \in C$

Proof. If $\phi_{g}=\lambda I$ for some $g \in C$ then, $\phi_{x}=\lambda I \forall x \in C$ since conjugating a scalar matrix does not change it. So, it suffices to prove that if $\phi_{g} \neq$ $\lambda I$ forsome $g \in C$ then, $\chi_{\phi}(g)=0$. We know that $h \chi(g) / d$ is an algebraic integer and $\chi(g)$ is an algebraic integer. Also, $\operatorname{gcd}(\mathrm{h}, \mathrm{d})=1$ so there exists integers $\mathrm{k}, \mathrm{j}$ such that $\mathrm{kh}+\mathrm{jd}=1$.Now,

$$
k\left(\frac{h \chi(g)}{d}\right)+j \chi(g)=\frac{\chi(g)}{d}=\alpha(\text { say })
$$

is an algebraic integer since set of all algebraic integers form a ring. Now, $\phi_{g}$ is diagonalizable with eigen values $\lambda_{1}, \lambda_{2}, \ldots, \lambda_{d}$. And, $\operatorname{chi}(g)=$ 
$\lambda_{1}+\lambda_{2}+\ldots .+\lambda_{d}$ that is, $\chi(g)$ is the sum of $\mathrm{d}$ nth roots of unity. Now $\left|\lambda_{1}+\ldots \lambda_{d}\right|<d$ since all eigen values are not same as we assumed that $\phi_{g} \neq \lambda I$. Thus, $|\chi(g)|<d$ that is, $\left|\frac{\chi(g)}{d}\right|<1$ that is, $|\alpha|<1$. And hence, $|\sigma(\alpha)|=\left|\sigma\left(\frac{\chi(g)}{d}\right)\right|<1$

Note that $q=\prod_{\sigma \in \Gamma} \sigma(\alpha)$ is an algebraic integer since $\alpha$ is an algebraic integer. It follows that

$$
|q|=\left|\prod_{\sigma \in \Gamma} \sigma(\alpha)\right|=\prod_{\sigma \in \Gamma}|\sigma(\alpha)|<1
$$

This shows that $q \in Q$ since it is an algebraic integer less than 1 . We know that a rational number is an algebraic integer iff it is an integer. This implies that $q \in \mathbb{Z}$ and $|q|<1$. Thus, $\mathrm{q}=0$. Therefore, $\sigma(\alpha)=0$ and hence, $\alpha=0$. Finally, we conclude that $\chi(g)=0$. Hence, proved.

Lemma 4.3.7. Let $G$ be a finite non abelian group. Let $C \neq\{1\}$ be the conjugacy class of $G$ such that $|C|=p^{t}$ where $p$ is prime and $t \geq 0$. Then, $G$ is not simple.

Proof. Suppose G is not simple. Then, let $\phi^{1}, \ldots, \phi^{s}$ be the complete set of representatives of irreducible representations of $\mathrm{G}$. Let $\chi_{1}, \ldots, \chi_{s}$ be their corresponding charcters and $d_{1}, \ldots d_{s}$ be their degrees. Without loss of generality, let $\phi^{1}$ be the trivial representation. Since G is simple, $\operatorname{Ker} \phi^{k}=\{1\} \forall k>1$ since if $K e r \phi^{k}=G$ then we have trivial representation. This shows that $\phi^{k}$ is one one for all $k>1$. Now, $\mathrm{G}$ is non abelian and $\mathbb{C}^{*}$ is abelian. This implies that $d_{k}>1 \forall k>1$. Since $\mathrm{G}$ is simple and non abelian this shows that $Z(G)=\{1\}$. Hence, $t>0$. Let $g \in C$ and $k>1$. Define

$$
Z_{k}=\left\{x \in G \mid \phi_{x}^{k} \text { is a scalar matrix }\right\}
$$

and

$$
H=\left\{\lambda I_{d_{k}} \mid \lambda \in \mathbb{C}^{*}\right\} .
$$

Note that $\mathrm{H}$ is a subgroup of $G L_{d_{k}}(\mathbb{C})$. In fact, $\mathrm{H}=\mathrm{Z}(\mathrm{G})$. Hence, $\mathrm{H}$ is normal. Now as $Z_{k}$ is inverse image of $\mathrm{H}$ under $\phi^{k}$ which is a homomorphism, $Z_{k}$ is a normal subgroup of G. Since, $d_{k}>1$ and $Z_{k} \neq G, Z_{k}=\{1\} \forall k>1$.

Suppose, $p \nmid d_{k}$ then, $\operatorname{gcd}\left(p, d_{k}\right)=1$. That is, $\chi_{\phi^{k}}(g)=0$. Let L be regular representation then,

$$
L \equiv d_{1} \phi^{1} \oplus \ldots \oplus d_{s} \phi^{s}
$$


Since, $g \neq 1, \chi_{L}(g)=d_{1} \chi_{1}+\ldots+d_{s} \chi_{s}=1+\sum_{k=2}^{s} d_{k} \chi_{k}=1+\sum_{p \mid d_{k}} d_{k} \chi_{k}=$ $1+p z$.

Also, $\chi_{L}(g)=0$. This implies that $1+\mathrm{pz}=0$. Thus, $1 / \mathrm{p}=-\mathrm{z}$ where $\mathrm{z}$ is algebraic integer. Hence, we get a contradiction since $\mathrm{z}$ has to be an integer. Therefore, our suppposition was wrong. Thus, G is not simple.

Theorem 4.3.8. Burnside's theorem: Let $G$ be a group of order $p^{a} q^{b}$ with primes $p, q$. Then, $G$ is not simple unless it is cyclic of prime order.

Proof. An abelian group is simple iff it is cyclic of prime order. Assume that $\mathrm{G}$ is non abelian. We know that a group of prime power order has non trivial centres. So, it is not simple. That is, here, in this case, if one of a or b is zero then, $\mathrm{G}$ is not simple. So, let $a, b \geq 1$.

By Sylow's theorem, ther exists a subgroup $\mathrm{H}$ of $\mathrm{G}$ of order $q^{b}$. Let $1 \neq g \in$ $Z(H)$.

Let $N_{G}(g)=\{x \in G \mid g x=x g\}$ is the normalizer of $\mathrm{g}$ in G.Now, since $g \in Z(H)$, g commutes with every element of $\mathrm{H}$. That is, every element of $\mathrm{H}$ commutes with g. Therefore, every element of $\mathrm{H}$ is in $N_{G}(g)$. Hence, $H \subseteq N_{G}(g)$.

Now $p^{a}=[G: H]=\left[G: N_{G}(g)\right]\left[N_{G}(g): H\right]$. This shows that $[G:$ $\left.N_{G}(g)\right]=p^{t}$ where $t>0$ but $|C l(g)|=$ conjugacyclassof $g=\left[G: N_{G}(g)\right]=$ $p^{t}$ where $t>0$. Hence, $\mathrm{G}$ is not simple by previous lemma. 


\section{Chapter 5}

\section{Group Actions and the Permutation Representations}

\subsection{Group Actions}

Definition 5.1.1. An action of a finite group $G$ on a finite set $X$ is a homomorphism $\sigma: G \rightarrow S_{X}$ where $S_{X}$ is the set of all bijections from $X$ to $X$. Moreover, $|X|$ is the degree of the action.

Definition 5.1.2. Let the action $\lambda$ be defined on $G$ itself that is, $\lambda: G \rightarrow S_{G}$ be an action. Then, $\lambda$ is called as regular action. And, the permutation $\tilde{\lambda}: G \rightarrow G L(\mathbb{C} G)$ is called the Regular Representation of $G$. We call the regular representation to be $L$.

Example 5.1.3. Let $G$ be a group and let $\lambda: G \rightarrow S_{G}$ be an action of $G$ on $G$ itself defined as, $\lambda_{g}(x)=g x$. Then, this action is called the Regular action of $G$ on $G$.

Definition 5.1.4. The subset $Y \subseteq X$ is called $G$-invariant if $\sigma_{g}(y) \in Y \forall y \in$ $Y$ and $g \in G$.

The set $X$ partitions into disjoint union of minimal G-invariant subsets called Orbits.

Definition 5.1.5. Orbit: Let $\sigma: G \rightarrow S_{X}$ be a group action. The orbit of $x \in X$ under $G$ is the set $G . x=\left\{\sigma_{g}(x) \mid g \in G\right\}$. 
An orbit is a G-invariant subset of $X$.

Definition 5.1.6. Transitive Action: A group action $\sigma: G \rightarrow S_{X}$ is said to be transitive if $\forall x, y \in X$, there exists $g \in G$ such that $\sigma_{g}(x)=y$.

In other words, an action is said to be transitive if there exists one orbit of $G$ on $X$.

Example 5.1.7. Let $H$ is a subgroup of $G$. Then, there exists an action $\sigma: G \rightarrow S_{G / H}$ by $\sigma_{g}(x H)=g x H$ known as the Coset Action of $G$. We note that this coset action is transitive.

Definition 5.1.8. 2-Transitive Action: Let $\sigma: G \rightarrow S_{X}$ be action of $G$ on $X$. Then it is said to be 2-transitive if for any given two pairs of distinct elements $x, y \in X, x^{\prime}, y^{\prime} \in X$, there exists a group element $g$ such that $\sigma_{g}(x)=x^{\prime}$ and $\sigma_{g}(y)=y^{\prime}$.

Note: The property of an action to be 2-transitive is a stronger property than an action to be transitive.

Example 5.1.9. Let $n \geq 2$. Then, the action of the symmteric group $S_{n}$ is 2-transitive on $\{i, 2, \ldots, n\}$. Consider two pairs of distinct elements say $i \neq j$ and $k \neq l$. Now, let $X=\{1,2, \ldots, n\}\{\tilde{i}, j\}$ and $Y=\{1,2, \ldots, n\}\{\tilde{\{}, l\}$. Then, $|X|=n-2=|Y|$. Now, we choose bijection $\alpha: X \rightarrow Y$. Define $\tau \in S_{n}$ by

$$
\tau(m)= \begin{cases}k & ; m=i \\ l & ; m=j \\ \alpha(m) & ; \text { otherwise }\end{cases}
$$

Then, $\tau(i)=k$ and $\tau(j)=l$. Hence, $S_{n}$ is 2-transitive.

Definition 5.1.10. Orbital: Let $\sigma: G \rightarrow S_{X}$ be a transitive group action. Define $\sigma^{2}: G \rightarrow S_{X \times X}$ defined by

$$
\sigma_{g}^{2}(x, x)=\left(\sigma_{g}\left(x_{1}\right), \sigma_{g}\left(x_{2}\right)\right)
$$

An orbit of $\sigma^{2}$ is known as the orbital of $\sigma$. The number of orbitals gives the rank of $\sigma$. 
Consider the set $\Delta=\{(x, x): x \in X\}$. We note that $\sigma_{g}^{2}(x, x)=$ $\left(\sigma_{g}(x), \sigma_{g}(x)\right)$. Now, if we consider $\sigma$ to be transitive then we conclude that $\Delta$ is an orbital of $\sigma$ called the trivial orbital or the diagonal orbital.

Proposition 5.1.11. Let $\sigma: G \rightarrow S_{X}$ be group action where $(|X| \geq 2)$ then, $\sigma$ is 2-transitive iff $\sigma$ is transitive and $\operatorname{rank}(\sigma)=2$.

Proof. If $\sigma$ is 2-transitive and $x, y \in X$ then we can choose $x^{\prime} \neq x$ and $y^{\prime} \neq y$ such that $\sigma_{g}(x)=x^{\prime}$ and $\sigma_{g}(y)=y^{\prime}$. So, $\sigma$ is transitive. Next, $(X \times X)$ other than $\Delta$ or the complement of $\Delta$ in $X \times X$ is the following set.

$$
\{(x, y) \mid x \neq y\}
$$

Therefore, $\Delta^{\complement}$ is an orbital iff for any two pairs $x \neq y$ and $x^{\prime} \neq y^{\prime}$ of distinct elements, there exists $g \in G$ such that $\sigma_{g}(x)=x^{\prime}$ and $\sigma_{g}(y)=y^{\prime}$ that is, $\sigma$ is 2-transitive. Consequently, $\operatorname{Rank}(\sigma)=2$ since there are two orbitals, $\Delta$ and $\Delta^{\complement}$.

Definition 5.1.12. Let $\sigma: G \rightarrow S_{X}$ be a group action. Then, for $g \in G$, we define,

$$
F i x(g)=\left\{x \in X \mid \sigma_{g}(x)=x\right\}
$$

to be the set of all fixed points with respect to $G$.

Similarly, Fix ${ }^{2}(g)$ is the set of fixed points with respect to $g$ on $X \times X$.

Proposition 5.1.13. Let $\sigma: G \rightarrow S_{X}$ be the group action. Then, Fix ${ }^{2}(g)=$ Fix $(g) \times F i x(g)$ and hence, $\left|F i x^{2}(g)\right|=|F i x(g)|^{2}$.

Proof. Let $(x, y) \in X \times X$. Then, $\sigma_{g}^{2}(x, y)=\left(\sigma_{g}(x), \sigma_{g}(y)\right)$. This implies that $(x, y)=\sigma_{g}^{2}(x, y)$ if and only if $\sigma_{g}(x)=x$ and $\sigma_{g}(y)=y$. We know that,

$$
F i x^{2}(g)=\left\{(x, y) \mid \sigma_{g}(x)=x, \sigma_{g}(y)=y\right\}
$$

Hence, $F_{i x}^{2}(g)=F i x(g) \times F i x(g)$.

Suppose we have an action $\sigma: G \rightarrow S_{n}$ and the standard representation of $S_{n}, \alpha: S_{n} \rightarrow G L_{n}(\mathbb{C})$ then, the composition $\alpha \circ \sigma: G \rightarrow G L_{n}(\mathbb{C})$ gives us the representation of $\mathrm{G}$. 
Definition 5.1.14. Let $\sigma: G \rightarrow S_{X}$ be a group action and hence $\tilde{\sigma}: G \rightarrow$ $G L(\mathbb{C} X)$ is the representation defined as,

$$
\tilde{\sigma}_{g}\left(\sum_{x \in X} c_{x} x\right)=\sum_{x \in X} c_{x} \sigma_{g}(x)=\sum_{y \in X} c_{\sigma_{g^{-1}}(y)} y
$$

This representation sigma is called the permutation representation associated to $\sigma$. In this representation, $x$ is mapped to $\sigma_{g}(x)$ and hence, the degree of representation $\tilde{\sigma}$ is equal to the degree of action $\sigma$.

Definition 5.1.15. Let $\lambda: G \rightarrow S_{G}$ be the regular action of $G$. Then,

$$
\tilde{\lambda}: G \rightarrow G L(\mathbb{C} G)
$$

is the regular representation of $G$.

Proposition 5.1.16. Let $\sigma: G \rightarrow S_{X}$ be a group action. Then, the representation $\tilde{\sigma}: G \rightarrow G L(\mathbb{C} X)$ is the permutation representation of $G$ and is a unitary representation.

Proposition 5.1.17. Let $\sigma: G \rightarrow S_{X}$ be a group action. Then, $\chi_{\tilde{\sigma}(g)}=$ $|F i x(g)|$.

Proof. Consider the set $X=\left\{x_{1}, x_{2}, \ldots, x_{n}\right\}$. Now, corresponding to this given action, we will have the representation $\tilde{\sigma}$ as defined in the proposition. Hence, $\left[\tilde{\sigma}_{g}\right]$ will be the matrix of $\tilde{\sigma}$ with respect to the basis X. We know that, $\tilde{\sigma}_{g}\left(x_{j}\right)=\sigma_{g}\left(x_{j}\right)$ and so,

$$
\left[\tilde{\sigma}_{g}\right]_{i j}= \begin{cases}1 & ; x_{i}=\sigma_{g}\left(x_{j}\right) \\ 0 & ; \text { otherwise }\end{cases}
$$

In particular,

$$
\left[\tilde{\sigma}_{g}\right]_{i i}= \begin{cases}1 & ; x_{i}=\sigma_{g}\left(x_{i}\right) \\ 0 & ; \text { otherwise }\end{cases}
$$

That is,

$$
\left[\tilde{\sigma}_{g}\right]_{i i}= \begin{cases}1 & ; x_{i} \in \text { Fix }(g) \\ 0 & ; \text { otherwise }\end{cases}
$$

Hence, $\chi_{\tilde{\sigma}}(g)=\operatorname{tr}\left(\left[\tilde{\sigma}_{g}\right]\right)=|F i x(g)|$. 
Definition 5.1.18. Fixed Subspace: Let $\phi: G \rightarrow G L(V)$ be a representation. Then,

$$
V^{G}=\left\{v \in V \mid \phi_{g}(v)=v \forall g \in G\right\}
$$

is called as the fixed subspace of $V$.

We note that $V^{G}$ is the G-invariant subspace.

Note: The subrepresentation $\phi \uparrow V^{G}$ is equivalent to $\operatorname{dim} V^{G}$ copies of the trivial representation.

Proposition 5.1.19. Let $\phi: G \rightarrow G L(V)$ be a representation. Let $\chi_{1}$ be the trivial character of $G$. Then, $\left\langle\chi_{\phi}, \chi_{1}\right\rangle=\operatorname{dim} V^{G}$.

Proof. Proof: Let $V=m_{1} V_{1} \oplus \ldots \oplus m_{s} V_{s}$ where $V_{1}, V_{2}, \ldots, V_{s}$ are the irreducible G-invariant subspaces of $\mathrm{V}$.

Without loss of Generality, assume that $V_{1}$ is equivalent to the trivial representation. Let $\phi^{i}=\phi \uparrow V_{i}$.

Let $v \in V$. Then, $v=v_{1}+v_{2}+\ldots v_{s}$ with $v_{i} \in m_{i} V_{i}$ and

$$
\phi_{g}(v)=\left(m_{1} \phi^{1}\right)_{g}\left(v_{1}\right)+\ldots+\left(m_{s} \phi^{s}\right)_{g}\left(v_{s}\right)
$$

and so, we conclude that,

$v \in V^{G}$ iff $v_{i} \in m_{i} V_{i}^{G} \forall 2 \leq i \leq s$. or we can say that,

$$
V^{G}=m_{1} V_{1} \oplus m_{2} V_{2}^{G} \oplus \ldots \oplus m_{s} V_{s}^{G}
$$

Let $i \geq 2$. Since, each $V_{i}$ is irreducible and not equivalent to the trivial representation and $V_{i}^{G}$ is G-invariant, $V_{i}^{G}=0$ and thus, $V^{G}=m_{1} V_{1}$.

Therefore, $\operatorname{dim} V^{G}=m_{1} \operatorname{dim} V_{1}$ and hence, $m_{1}$ is the dimension of $V_{g}$ where $m_{1}$ is the multiplicity of trivial representation in $\phi$

Proposition 5.1.20. Let $\sigma: G \rightarrow S_{X}$ be a group action. Let $O_{1}, O_{2}, \ldots, O_{m}$ be the orbits of $G$ on $X$ and define $v_{i}=\sum_{x \in O_{i}} x$. Then, $v_{1}, v_{2}, \ldots, v_{m}$ is the basis for $\mathbb{C} X^{G}$ and hence, $\operatorname{dim} \mathbb{C} X^{G}$ is the number of orbits of $G$ on $X$.

Proof. We need to show that $v_{1}, v_{2}, \ldots . v_{m}$ is the linearly independent set and spans $\mathbb{C} X^{G}$. First to show linear independence, we observe that,

$$
\tilde{\sigma}_{g}\left(v_{i}\right)=\sum_{x \in O_{i}} \sigma_{g}(x)=\sum_{y \in O_{i}} y=v_{i}
$$


Form this we conclude that $v_{1}, v_{2}, \ldots, v_{m}$ belongs to the fixed subspace $X^{G}$ and hence, $v_{1}, v_{2}, \ldots, v_{m} \in \mathbb{C} X^{G}$ Now, since the orbits are disjoint and we know that if $v_{i}=\sum_{x \in O_{i}} c_{i} x$ and $v_{j}=\sum_{x \in O_{j}} c_{j} x$ are the two formal sums where $c_{i}$ and $c_{j}$ are the coefficients then

$$
\left\langle v_{i}, v_{j}\right\rangle=\sum c_{i} \overline{c_{j}}
$$

As here, we have all coefficients in the formal sum to be 1, therefore,

$$
\left\langle v_{i}, v_{j}\right\rangle= \begin{cases}\left|O_{i}\right| & ; i=j \\ 0 & ; i \neq j\end{cases}
$$

which shows that $\left\{v_{1}, v_{2}, \ldots, v_{m}\right\}$ is an orthogonal set of non zero vectors and hence linearly independent. Now it remains to prove that this spans $\mathbb{C} X^{G}$. For this, suppose, $v=\sum_{x \in X} c_{x} x \in \mathbb{C} X^{G}$. We first show that if some elememt $z \in G$.y then, $c_{y}=c_{z}$. Let $z \in G$.y then, $z=\sigma_{g}(y)$ then,

$$
\sum_{x \in X} c_{x} x=v=\tilde{\sigma}_{g}(v)=\sum_{x \in X} c_{x} \sigma_{g}(x)
$$

Now the coefficient of $\mathrm{z}$ in left hand side is $c_{z}$ and the coefficient of $\mathrm{z}$ in right hand side is $c_{y}$ since $z=\sigma_{g}(y)$. Therefore, $c_{z}=c_{y}$.

This implies that there exist $c_{1}, c_{2}, \ldots, c_{m} \in \mathbb{C}$ such that $c_{x}=c_{i} \forall x \in O_{i}$. Thus,

$$
v=\sum_{x \in X} c_{x} x=\sum_{i=1}^{m} \sum_{x \in O_{i}} c_{x} x=\sum_{i=1}^{m} c_{i} \sum_{x \in O_{i}} x=\sum_{i=1}^{m} c_{i} v_{i}
$$

Hence, $v_{1}, v_{2}, \ldots, v_{m}$ spans $\mathbb{C} X^{G}$ and hence is the basis for the same.

Corollary 5.1.1. Burnside's lemma: Let $\sigma: G \rightarrow S_{X}$ be a group action and let $m$ be the number of orbits of $G$ on $X$. Then, $m=\frac{1}{O(G)} \sum_{g \in G} \mid$ Fix $(g) \mid$

Proof. Let $\chi_{1}$ be the trivial character of G. We know that the multiplicity of the trivial representation in $\tilde{\sigma}, m=\left\langle\chi_{\tilde{\sigma}}, \chi_{1}\right\rangle=\operatorname{dim} V^{G}$.

And also, we know that

$$
\left\langle\chi_{\tilde{\sigma}}, \chi_{1}\right\rangle=\frac{1}{O(G)} \sum_{g \in G} \chi_{\tilde{\sigma}}(g) \overline{\chi_{1}(g)}=\frac{1}{O(G)} \sum_{g \in G}|F i x(g)|
$$

Hence, $m=\frac{1}{O(G)} \sum_{g \in G}|F i x(g)|$. 
Corollary 5.1.2. Let $\sigma: G \rightarrow S_{X}$ be a transitive group acion. Then,

$$
\operatorname{rank}(\sigma)=\frac{1}{O(G)} \sum_{g \in G}|\operatorname{Fix}(g)|^{2}=\left\langle\chi_{\tilde{\sigma}}, \chi_{\tilde{\sigma}}\right\rangle
$$

Proof. We know that thr rank of the action $\sigma$ is equal to the number of orbits of $\sigma^{2}$ on $X \times X$. And, the number of fixed points of $g$ on $X \times X$ is $\mid$ Fix $\left.(g)\right|^{2}$.

From the consequence of Burnside's lemma, we can conclude the first equality. For the second one,

$$
\begin{aligned}
\left\langle\chi_{\tilde{\sigma}}, \chi_{\tilde{\sigma}}\right\rangle & =\frac{1}{O(G)} \sum_{g \in G}|F i x(g)| \overline{|F i x(g)|} \\
& =\frac{1}{O(G)} \sum_{g \in G}|F i x(g)|^{2} .
\end{aligned}
$$

Hence, by this we get the second equality.

Suppose $\sigma: G \rightarrow S_{X}$ be a transitive group action. Let $v_{0}=\sum_{x \in X} x$. Then, we prove that $\mathbb{C} X^{G}=\mathbb{C} v_{0}$.

We know that $\mathbb{C} X^{G}=\left\{x \in X \mid \sigma_{g}(x)=x\right\}$ and $\mathbb{C} v_{0}=\operatorname{span}\left\{\sum_{x \in X} x\right\}$. Now, let $v \in \mathbb{C} v_{0}$ that is, $v=\sum_{x \in X} c x$. now,

$$
\tilde{\sigma}_{g}\left(\sum_{x \in X} c x\right)=\sum_{x \in X} \sigma_{g}(c x)=\sum_{x \in X} c \sigma_{g}(x)=\sum_{y \in X} c y=v
$$

since the action is transitive and the representation $\tilde{\sigma}$ is a unitary representation. This shows that $v \in \mathbb{C} X^{G}$ and hence $\mathbb{C} v_{0} \subseteq \mathbb{C} X^{G}$.

Now, we show the other part. We know that the orbit of $\mathrm{x}$ is,

$$
G . x=\left\{\sigma_{g}(x) \mid g \in G\right\}
$$

now, since the action is given to be transitive, we have $G . x=X$. Hence, by previous theorem, it follows that $v_{0}$ is a basis for $\mathbb{C} X^{G}$.

Hence, $\mathbb{C} X^{G}=\mathbb{C} v_{0}$. Now let $V_{0}=\mathbb{C} v_{0}^{\perp}$ is a G-invariant subspace.

We define $\mathbb{C} v_{0}$ to be trace of $\sigma$ and $V_{0}$ to be augmentation of $\sigma$. Let $\tilde{\sigma}^{\prime}$ be te restiction of $\tilde{\sigma}$ to $V_{0}$. Hence, $\tilde{\sigma}^{\prime}$ is the augmentation representation associated to $\sigma$. 
As $\mathbb{C} X=V_{0} \oplus \mathbb{C} v_{0}$, we have,

$$
\chi_{\tilde{\sigma}}=\chi_{\tilde{\sigma}}^{\prime}+\chi_{1}
$$

where $\chi_{1}$ is the trivial character.

Theorem 5.1.21. Let $\sigma: G \rightarrow S_{X}$ be a transitive group action. Then, the augmentation representation $\tilde{\sigma}^{\prime}$ is irreducible iff $\sigma$ is 2-transitive on $X$.

Proof. We know that $\sigma$ is 2 -transitive iff $\operatorname{rank}(\sigma)=2$. Let $\chi_{1}$ be the trivial character of G. Then,

$$
\left\langle\chi_{\tilde{\sigma}^{\prime}}, \chi_{\tilde{\sigma}^{\prime}}\right\rangle=\left\langle\chi_{\tilde{\sigma}}-\chi_{1}, \chi_{\tilde{\sigma}^{\prime}}-\chi_{1}\right\rangle=\left\langle\chi_{\tilde{\sigma}}, \chi_{\tilde{\sigma}}\right\rangle-\left\langle\chi_{\tilde{\sigma}}, \chi_{1}\right\rangle-\left\langle\chi_{1}, \chi_{\tilde{\sigma}}\right\rangle+\left\langle\chi_{1}, \chi_{1}\right\rangle
$$

Now, since $\sigma$ is transitive, $\left\langle\chi_{\tilde{\sigma}}, \chi_{1}\right\rangle=1$ and $\left\langle\chi_{1}, \chi_{\tilde{\sigma}}\right\rangle=1$. Also, $\left\langle\chi_{1}, \chi_{1}\right\rangle=1$. This implies that,

$$
\left\langle\chi_{\tilde{\sigma}^{\prime}}, \chi_{\tilde{\sigma}^{\prime}}\right\rangle=\operatorname{rank}(\sigma)-1
$$

and so, $\chi_{\tilde{\sigma}^{\prime}}$ is irreducible character iff $\operatorname{rank}(\sigma)=2$ that is, iff $\sigma$ is 2 - transitive on $\mathrm{X}$.

\subsection{The Centralizer Algebra}

\section{Some Notations:}

Let $\sigma: G \rightarrow S_{X}$ is a transitive group action. Our aim is to study the set $\operatorname{Hom}_{G}(\tilde{\sigma}, \tilde{\sigma})$ which is the set of all morphisms from $\tilde{\sigma}$ to iself. That is , it is the set of all invertible maps $\mathrm{T}$ such that $T \tilde{\sigma}_{g}=\tilde{\sigma}_{g} T$.

Let us consider the set, $X=\left\{x_{1}, x_{2}, \ldots, x_{n}\right\}$. Let $\phi: G \rightarrow G L_{n}(\mathbb{C})$ be defined as, $\phi_{g}=\left[\tilde{\sigma}_{g}\right]_{X}$.

Then, $\phi \sim \tilde{\sigma}$ and so, $\operatorname{Hom}_{G}(\tilde{\sigma}, \tilde{\sigma}) \equiv \operatorname{Hom}_{G}\left(\phi_{g}, \phi_{g}\right)$.

$$
\begin{gathered}
\operatorname{Hom}_{G}(\phi, \phi)=\left\{A \in M_{n}(\mathbb{C}) \mid A \phi_{g}=\phi_{g} A \forall g \in G\right\} \text { that is exactly the set } \\
\left\{A \in M_{n}(\mathbb{C}) \mid \phi_{g} A \phi_{g}^{-1}=A \forall g \in G\right\}
\end{gathered}
$$

We denote $\operatorname{Hom}_{G}(\phi, \phi)$ as $C(\sigma)$ known as the Centralizer algebra of $\sigma$.

Proposition 5.2.1. $C(\sigma)$ is a unital subring of $M_{n}(\mathbb{C})$. 
Proof. We note that, $\phi_{g} I_{n} \phi_{g}^{-1}=I_{n} \forall g \in G$. Let $A, B \in C(\sigma)$ then,

$$
\phi_{g}(A+B) \phi_{g}^{-1}=\phi_{g} A \phi_{g}^{-1}+\phi_{g} B \phi_{g}^{-1}=A+B \forall g \in G
$$

Similarly, $\phi_{g}(A B) \phi_{g}^{-1}=\phi_{g} A \phi_{g}^{-1} \phi_{g} B \phi_{g}^{-1}=A B$.

This shows that $C(\sigma)$ is a unital subring of $M_{n}(\mathbb{C})$.

Next, we show that, $\operatorname{dim}(C(\sigma))=\operatorname{rank}(\sigma)$. The proof goes as follows.

Let $V=M_{n}(\mathbb{C})$ and $\tau: G \rightarrow G L(V)$ by $\tau_{g}(A)=\phi_{g} A \phi_{g}^{-1}$ be a representation.Then,

$$
V^{G}=\left\{A \in M_{n}(\mathbb{C}) \mid \phi_{g} A \phi_{g}^{-1}=A \forall g \in G\right\}=C(\sigma)
$$

For the next proposition we retain the same notations described right now. And also, consider $\sigma^{2}: G \rightarrow S_{X \times x}$

Proposition 5.2.2. Define a map $T: M_{n}(\mathbb{C}) \rightarrow \mathbb{C}(X \times X)$ by

$$
T\left(a_{i j}\right)=\sum_{i, j=1}^{n} a_{i j}\left(x_{i}, x_{j}\right)
$$

Then, $T$ is an equivalence between $\tau$ and $\sigma^{2}$.

Proof. The defined map $\mathrm{T}$ is clearly linear and invertible with the inverse,

$$
\sum_{i, j=1}^{n} a_{i j}\left(x_{i}, x_{j}\right) \longmapsto\left(a_{i j}\right)
$$

Let $g \in G$ and let $A=\left(a_{i j}\right) \in M_{n}(\mathbb{C})$ and $B=\left(b_{i j}\right)=\tau_{g} A=\phi_{g} A \phi_{g}^{-1}$. Next, we define action $\gamma: G \rightarrow S_{n}$ as

$$
\sigma_{g}\left(x_{i}\right)=x_{\gamma_{g}(i)} \text { for } g \in G
$$

As,

$$
\phi(g)_{i k}= \begin{cases}1 & ; x_{i}=\sigma_{g}\left(x_{k}\right) \\ 0 & ; \text { otherwise }\end{cases}
$$

and,

$$
\phi\left(g^{-1}\right)_{l j}= \begin{cases}1 & ; x_{l}=\sigma_{g^{-1}}\left(x_{j}\right) \\ 0 & ; \text { otherwise }\end{cases}
$$


we have,

$$
b_{i j} \sum_{k=1, l=1}^{n} \phi(g)_{i k} a_{k l} \phi\left(g^{-1}\right)_{l j}=a_{\gamma_{g^{-1}(i)}, \gamma_{g^{-1}}(j)}
$$

Therefore,

$$
\begin{aligned}
T \tau_{g} A & =\sum_{i, j=1}^{n} b_{i j}\left(x_{i}, x_{j}\right) \\
& =\sum_{i, j=1}^{n} a_{\gamma_{g^{-1}(i)}, \gamma_{g^{-1}}(j)}\left(x_{i}, x_{j}\right) \\
& =\sum_{i, j=1}^{n} a_{k l}\left(\sigma_{g}\left(x_{k}\right), \sigma_{g}\left(x_{l}\right)\right) \\
& =\sum_{i, j=1}^{n} a_{i j}\left(\sigma_{g}\left(x_{i}\right), \sigma_{g}\left(x_{j}\right)\right) \\
& =\sum_{i, j=1}^{n} a_{i j} \sigma_{g}^{2}\left(x_{i}, x_{j}\right) \\
& =\underset{\sigma_{g}^{2}}{T} A
\end{aligned}
$$

Hence, $\mathrm{T}$ is an equivalence between $\tau$ and $\sigma^{2}$.

Now, as our motive was to determine the dimension of $C(\sigma)$, so, must be thinking that what can be the basis for $C(\sigma)$.

Let $\mathrm{O}$ be an orbital of $\sigma$ that is, orbital of $\sigma^{2}$.

Define $A(O) \in M_{n}(\mathbb{C})$ by

$$
A(O)_{i j}= \begin{cases}1 & ;\left(x_{i}, x_{j}\right) \in O \\ 0 & ; \text { otherwise }\end{cases}
$$

Following corollary describes the basis for $C(\sigma)$.

Corollary 5.2.1. Let $\sigma: G \rightarrow S_{X}$ be a transitive group action. Let $O_{1}, O_{2}, \ldots, O_{r}$ be the orbitals of $\sigma$ where $r$ is the rank of $\sigma$. Then, the set $\left\{A\left(O_{1}\right), A\left(O_{2}\right), \ldots, A\left(O_{r}\right)\right\}$ is a basis for $C(\sigma)$ and consequently $\operatorname{dim}(C(\sigma))=\operatorname{rank}(\sigma)$

Proof. Proof: By the proposition done earlier, we know that,

$$
v_{k}=\sum_{\left.\left(x_{i}, x_{j}\right) \in\right)(k)}\left(x_{i}, x_{j}\right)
$$


is a basis for $\mathbb{C}(X \times X)^{G}$.

Clearly, $A\left(O_{k}\right)=T^{-1} v_{k}$ where, $T: M_{n}(\mathbb{C}) \rightarrow \mathbb{C}(X \times X)$ defined as,

$$
T\left(a_{i j}\right)=\sum_{i, j=1}^{n} a_{i j}\left(x_{i}, x_{j}\right)
$$

Now, as $\mathrm{T}$ is an equivalence from $M_{n}(\mathbb{C})$ and $\mathbb{C}(X \times X)$, so it restricts its equivalence to $M_{n}(\mathbb{C})=C(\sigma)$ and $\mathbb{C}(X \times X)$.

Therefore, $\left\{A\left(O_{1}\right), \ldots, A\left(O_{r}\right)\right\}$ is a basis for $C(\sigma)$.

Hence, $\operatorname{dim}(C(\sigma))=\operatorname{rank}(\sigma)$.

\subsection{Gelfand Pair}

Definition 5.3.1. Let $G$ be a group. Let $H$ be the subgroup of $G$. Let $\sigma$ : $G \rightarrow S_{G / H}$ be the coset action. Then, $(G, H)$ is said to be Gelfand Pair if the centralizer algebra $C(\sigma)$ is commutative.

Example 5.3.2. Let $G$ be a group. Let $H$ be its subgroup such that $H=\{1\}$. Let $\lambda: G \rightarrow S_{G}$ be the regular action of $G$ and hence, lambda $: G \rightarrow G L(\mathbb{C} G)$ is the regular representation of $G$ say, $L$.

Our claim is $C(\lambda)$ is isomorphic to $L(G)$.

Let $T \in C(\lambda)$ and define $f_{T}: G \rightarrow \mathbb{C}$ by,

$$
T .1=\sum_{x \in G} f_{T}\left(x^{-1}\right) x
$$

We show that the map $\psi: C(\lambda) \rightarrow L(G)$ defined as,

$$
T \longmapsto f_{T}
$$

is an isomorphism.

First, note that, since $T \in C(\lambda)$ that is, $T \in \operatorname{Hom}_{G}(L, L)$ so, $L_{g} T=T L_{g}$.

Now, for $g \in G$,

$$
T_{g}=T L_{g} \cdot 1=L_{g} T .1=L_{g} \sum_{x \in G} f_{T}\left(x^{-1}\right) x .
$$


Therefore, $T$ is determined by $f_{T}$ and hence, this shows that, $\psi$ is one one. Next, we need to show that $\psi$ is onto.

Let $f: G \rightarrow \mathbb{C}$ be a function in $L(G)$. Then, we can define $T \in \operatorname{End}(\mathbb{C} G)$ on the basis as,

$$
T_{g}=L_{g} \sum_{x \in G} f\left(x^{-1}\right) x
$$

Now, if $g, y \in G$ then,

$$
T L_{y} g=T_{y g}=L_{y g} \sum_{x \in G} f\left(x^{1}\right) x=L_{y} T_{g}
$$

So, $T \in C(\lambda)$. Also, $T .1=\sum_{x \in G} f\left(x^{-1}\right) x$ and so, $f_{T}=f$. Hence, $\psi$ is onto. Next, we show that $\psi$ is homomorphism.

Let $T_{1}, T_{2} \in C(\lambda)$.

$$
\begin{aligned}
T_{1} T_{2} .1 & =T_{1} \sum_{x \in G} f_{T_{2}}\left(x^{-1}\right) x \\
& =\sum_{x \in G} f_{T_{2}}\left(x^{-1}\right) T_{1} L_{x} 1 \\
& =\sum_{x \in G} f_{T_{2}}\left(x^{-1}\right) L_{x} \sum_{x \in G} f_{T_{1}}\left(y^{-1}\right) y \\
& =\sum_{x, y \in G} f_{T_{1}}\left(y^{-1}\right) f_{T_{2}}\left(x^{-1}\right) x y
\end{aligned}
$$

Let $g=x y$ and $u=x^{-1}$ then, $y^{-1}=g^{-1} u^{-1}$. This shows that,

$$
\begin{aligned}
T_{1} T_{2} .1 & =\sum_{g \in G} \sum_{u \in G} f_{T_{1}}\left(g^{-1} u^{-1}\right) f_{T_{2}}(u) g \\
& =\sum_{g \in G} f_{T_{1}} * f_{T_{2}}\left(g^{-1}\right) g .
\end{aligned}
$$

This implies that, $f_{T_{1} T_{2}}=f_{T_{1}} * f_{T_{2}}$.

Hence, $\psi$ is homomorphism. Therefore, we conclude that, $\psi$ is an isomorphism.

Now, as a consequence,

$(G,\{1\})$ is a Gelfand Pair

$\Longleftrightarrow C(\lambda)$, the centralizer algebra is commuattive that is, 
$\Longleftrightarrow L(G)$ is commutative.

$\Longleftrightarrow L(G)=Z(L(G))$.

We know that, $\operatorname{dim}(Z(L(G)))=|C l(G)|$ and $\operatorname{dim}(L(G))=|G|$.

So, $(G,\{1\})$ is a Gelfand Pair.

$\Longleftrightarrow|C l(G)|=|G|$.

$\Longleftrightarrow G$ is abelian.

Therefore, $(G,\{1\})$ is a Gelfand Pair $\Longleftrightarrow G$ is abelian.

Note: $(\mathrm{G}, \mathrm{H})$ is a Gelfand Pair iff $\tilde{\sigma}$ is multiplicity free that is, each irreducible constituent of sigma has multiplicity one.

Definition 5.3.3. Let $\sigma: G \rightarrow S_{X}$ be a transitive action. Then, to each orbital $O$ of $\sigma$, we can associate its transpose,

$$
O^{T}=\left\{\left(x_{1}, x_{2}\right) \in X \times X \mid\left(x_{2}, x_{1}\right) \in O\right\}
$$

which is also an orbital since the action is transitive.

Definition 5.3.4. $O$ is symmetric orbital if $O=O^{T}$.

Example 5.3.5. The diagoanl orbital $\Delta$ is symmetric.

Note that, $A\left(O^{T}\right)=A(O)^{T}$.

Hence, $\mathrm{O}$ is symmetric iff the matrix $\mathrm{A}(\mathrm{O})$ is symmetric.

Definition 5.3.6. Symmetric Gelfand Pair: Let $G$ be a group and $H$ be its subgroup with corresponding group action $\sigma: G \rightarrow S_{G / H}$. Then, $(G, H)$ is called a symmetric Gelfand Pair if each orbital of $\sigma$ is symmetric.

Example 5.3.7. Let $H$ be a subgroup of a group $G$. Let action of $G$ on $G / H$ is 2-transitive. Then, orbitals are, $\Delta$ and $\Delta^{\complement}$ in $G / H \times G / H$. Clearly, each one of them is symmetric.

Example 5.3.8. Let $n \geq 2$. Let $N$ be the set of all 2-element subsets of $\{1,2, \ldots, n\}$. Then, $S_{n}$ acts on $N$ as follows.

Define, $\tau: S_{n} \rightarrow S_{N}$ by,

$$
\tau_{\sigma}(\{i, j\})=\{\sigma(i), \sigma(j)\} .
$$

Note that this action is clearly transitive since $S_{n}$ is 2-transitive on $\{1,2, \ldots, n\}$. Let $H$ be the stabilizer of $\{n-1, n\}$ in $S_{n}$. 
So, $H$ is the internal direct product of $S_{n-2}$ and $S_{\{n-1, n\}}$ and hence, $H \equiv$ $S_{n-2} \times S_{2}$.

Let us recall the definition of internal direct product.

A group $G$ is the internal product of two subgroups $N_{1}, N_{2}$ if the following conditions hold:

(i) $N_{1}, N_{2}$ are both normal subgroups and,

(ii) $N_{1} \cap N_{2}=\{0\}$ and $N_{1} N_{2}=G$.

Equivalently, $G$ is the internal direct product of $N_{1}, N_{2}$ if every element of $N_{1}$ commutes with every element of $N_{2}$ that is, $N_{1} \subset$ centralizer $\left(N_{2}\right)$.

Getting back to the example, we find that the action of $S_{n}$ on $N$ can be identified with the action of $S_{n}$ on $S_{n} / H$.

Now, let $O$ be a non trivial orbital then, any element of $O$ is of the form, $(\{i, j\},\{k, l\})$ where $\{i, j\} \neq\{k, l\}$. Now we have two cases:

Case(i) : If all are distinct, then, the permutaion of $S_{n},(i k)(j l)$ takes this element to $(\{k, l\},\{i, j\})$ so, $O$ is symmetric.

Case(ii) : Suppose $i=k$. Then, the permutation $(i k)(j l)$ becomes $(j l)$ which takes this element to $(\{i, l\},\{i, j\},\{i, j\})$. Therefore, $O$ is again symmetric since $i=k$.

So, $\left(S_{n}, H\right)$ is a Symmetric Gelfand Pair.

Lemma 5.3.9. Let $R$ be a subring of $M_{n}(\mathbb{C})$ consisting of symmetric matrices. Then $R$ is commutative.

Proof. Let $A, B \in R$. Then, $A B \in R$ and hence,

$$
A B=(A B)^{T}=B^{T} A^{T}=B A .
$$

Hence, $\mathrm{R}$ is commutative.

Theorem 5.3.10. Let $(G, H)$ be symmetric Gelfand Pair. Then, $(G, H)$ is a Gelfand Pair.

Proof. Let $\sigma: G \rightarrow S_{G / H}$ be an action. Let $O_{1}, O_{2}, \ldots, O_{r}$ be the orbitals of $\sigma$.

Since, each $O_{i}$ is symmetric, each $A\left(O_{i}\right)$ is symmetric for $i=1,2, \ldots, r$. Since, symmetric matrices form a vector subspace of $M_{n}(\mathbb{C})$ and

$$
\left\{A\left(O_{1}\right), A\left(O_{2}\right), \ldots, A\left(O_{r}\right)\right\}
$$


is a basis for $C(\sigma)$ (by corollary done before).

This shows that $C(\sigma)$ consists of symmetric matrices. So, $C(\sigma)$ is commutative by previous lemma. Hence, $(\mathrm{G}, \mathrm{H})$ is a Gelfand Pair. 


\section{Bibliography}

[1] B. Simon, Representations of finite and compact groups, Graduate Studies in Mathematics, 10, American Mathematical Society, Providence, RI, 1996. MR1363490

[2] B. Steinberg, Representation theory of finite groups, Universitext, Springer, New York, 2012. MR2867444

[3] J.-P. Serre, Représentations linéaires des groupes finis, third revised edition, Hermann, Paris, 1978. MR0543841 